\title{
Thioarylative Radical Cyclization of Yne-Dienone
}

Rajendra K. Mallick, ${ }^{\dagger}$ Shubham Dutta, ${ }^{\dagger}$ Rajeshwer Vanjari, ${ }^{\dagger}$ Arnaud Voituriez, ${ }^{\ddagger}$ and Akhila K. Sahoo*广

'School of Chemistry, University of Hyderabad, Hyderabad-500046, India

${ }^{¥}$ Institut de Chimie des Substances Naturelles (ICSN), CNRS, 91198 Gif-sur-Yvette Cedex, France

akhilchemistry12@gmail.comorakssc@uohyd.ac.in

SUPPORTING INFORMATION

\begin{tabular}{|l|c|}
\hline Table of Contents & Page \\
\hline X-ray Crystallography Information & S2-S4 \\
\hline References & S5 \\
\hline NMR data & S6-S76 \\
\hline
\end{tabular}




\section{X-ray crystallography of $3 \mathbf{a}^{1,2}$}

Single crystal X-ray data for the compound 3a was collected using the Bruker D8 Quest CMOS detector system $[\lambda(\mathrm{Mo}-\mathrm{K} \alpha)=0.71073 \AA]$ at $298 \mathrm{~K}$, graphite monochromator with a $\omega$ scan width of $0.3 \mathrm{o}$, crystal-detector distance $60 \mathrm{~mm}$, collimator $0.5 \mathrm{~mm}$. The SMART software was used for the intensity data acquisition and the SAINTPLUS Software was used for the data extraction. In each case, absorption correction was performed with the help of SADABS program, an empirical absorption correction using equivalent reflections was performed with the program. The structure was solved using SHELXS-97, OLEX, and full-matrix least-squares refinement against F2 was carried out using SHELXL-97. All non-hydrogen atoms were refined anisotropically. Aromatic and methyl hydrogens were introduced on calculated positions and included in the refinement riding on their respective parent atoms. 


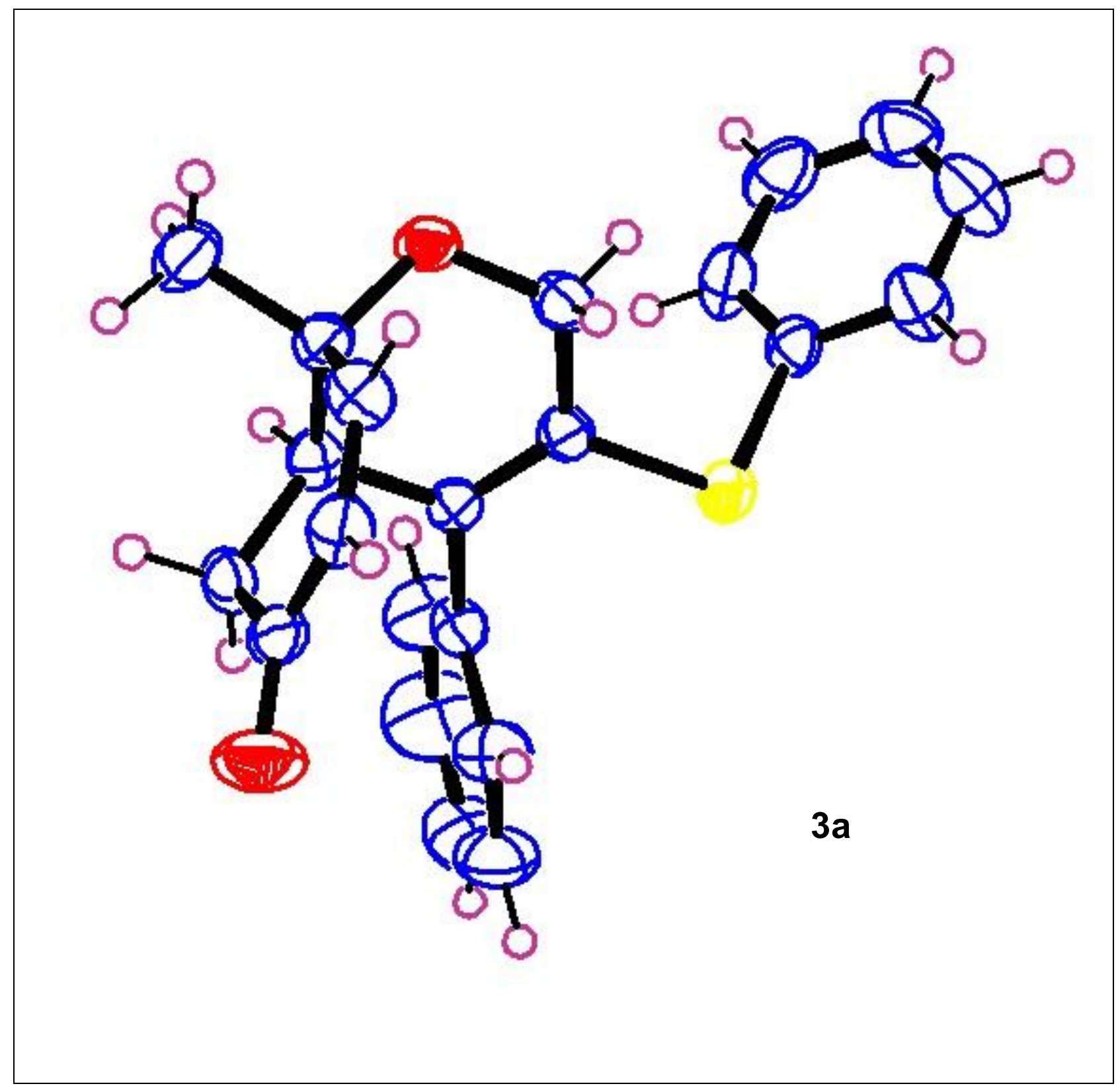

Figure S1. ORTEP-3 diagram of compound 3a; ORTEP Drawing of the compound 3a shows ellipsoid contour at the $50 \%$ probability level. Single crystal was grown by the slow evaporation of the solution of compound 3a in chloroform and $n$-hexane (1:1) mixture. Carbon (blue), Hydrogen (violet red), Oxygen (red), Sulphur (yellow). 
Table S1. Crystallographic Data for Compound 3a.

\begin{tabular}{|c|c|}
\hline Compound & 3a \\
\hline formula & $\mathrm{C}_{22} \mathrm{H}_{20} \mathrm{O}_{2} \mathrm{~S}$ \\
\hline MW & 348.44 \\
\hline crystal system & triclinic \\
\hline space group & $\mathrm{P}-1$ \\
\hline $\mathrm{T}[\mathrm{K}]$ & $297 \mathrm{~K}$ \\
\hline $\mathrm{a}[\AA]$ & 11.189(3) \\
\hline $\mathrm{b}[\AA]$ & $11.474(2)$ \\
\hline$c[\AA]$ & $14.860(3)$ \\
\hline$\alpha\left[{ }^{\circ}\right]$ & 84.479(9) \\
\hline$\beta\left[^{\circ}\right]$ & $87.969(10)$ \\
\hline$\gamma\left[^{\circ}\right]$ & $77.882(9)$ \\
\hline$V\left[\AA^{3}\right]$ & 1856.3(7) \\
\hline$Z$ & 4 \\
\hline$\rho_{\text {calcd }}\left[\mathrm{g} \mathrm{cm}^{-3}\right]$ & 1.247 \\
\hline$\mu\left[\mathrm{mm}^{-1}\right]$ & 0.186 \\
\hline total reflns & 8731 \\
\hline unique reflns & 8632 \\
\hline observed & 4762 \\
\hline $\mathrm{R}_{1}[\mathrm{I}>2 \sigma(\mathrm{I})]$ & 0.0609 \\
\hline wR2 [all] & $0.1335(8632)$ \\
\hline GOF & 1.063 \\
\hline Diffractometer & Bruker D8 Quest CCD \\
\hline CCDC Number & 1897537 \\
\hline
\end{tabular}




\section{References}

1. Dolomanov, O. V.; Blake, A. J.; Champness, N. R.; Schroder, M. OLEX: new software for visualization and analysis of extended crystal structures. J. Appl. Cryst. 2003, 36, 1283.

2. (a) SMART (version 5.625), SHELX-TL (version 6.12), Bruker AXS Inc.Madison, WI, 2000.

(b) G. M. Sheldrick, SHELXS-97, University of Gottingen, Germany, 1997. 

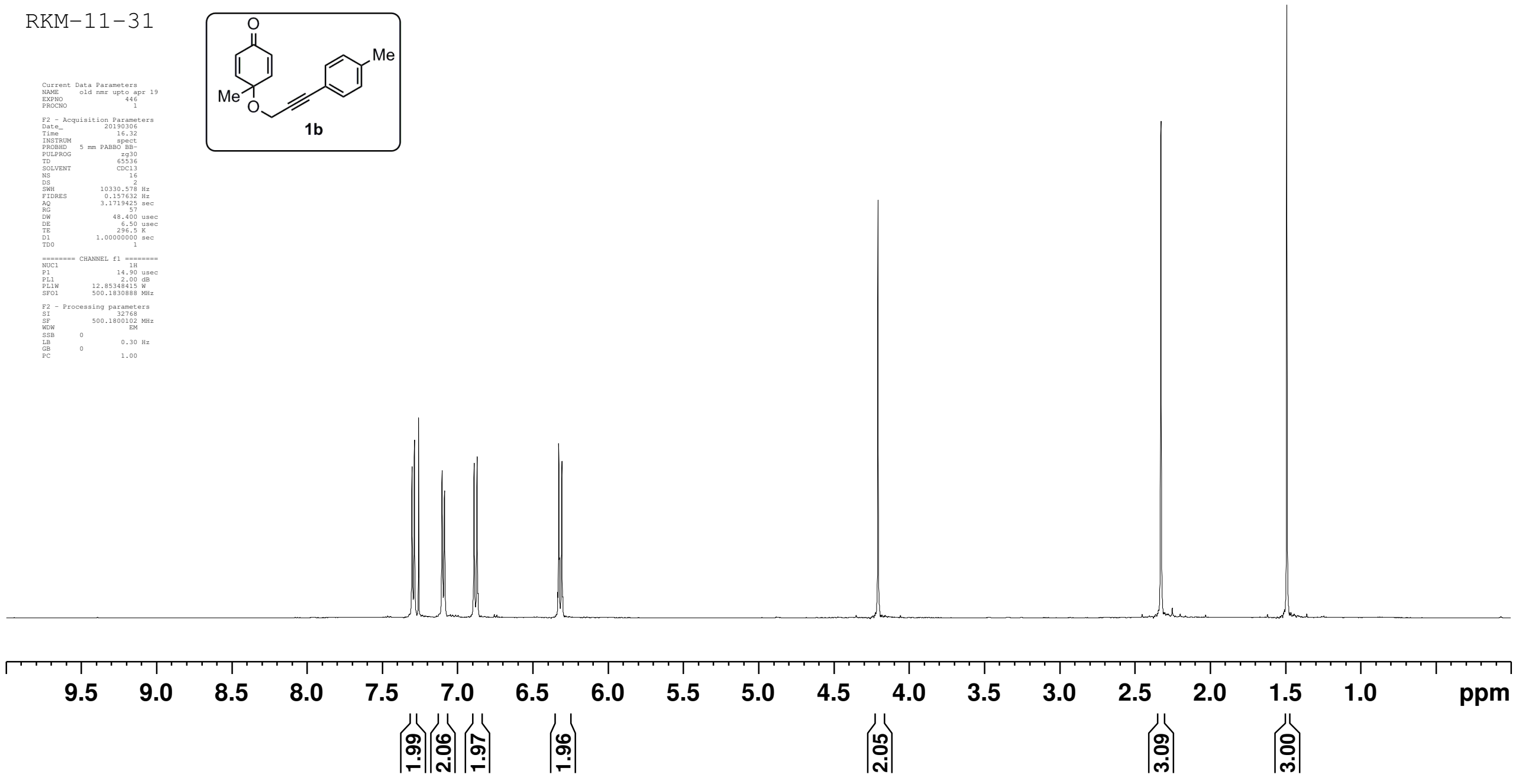


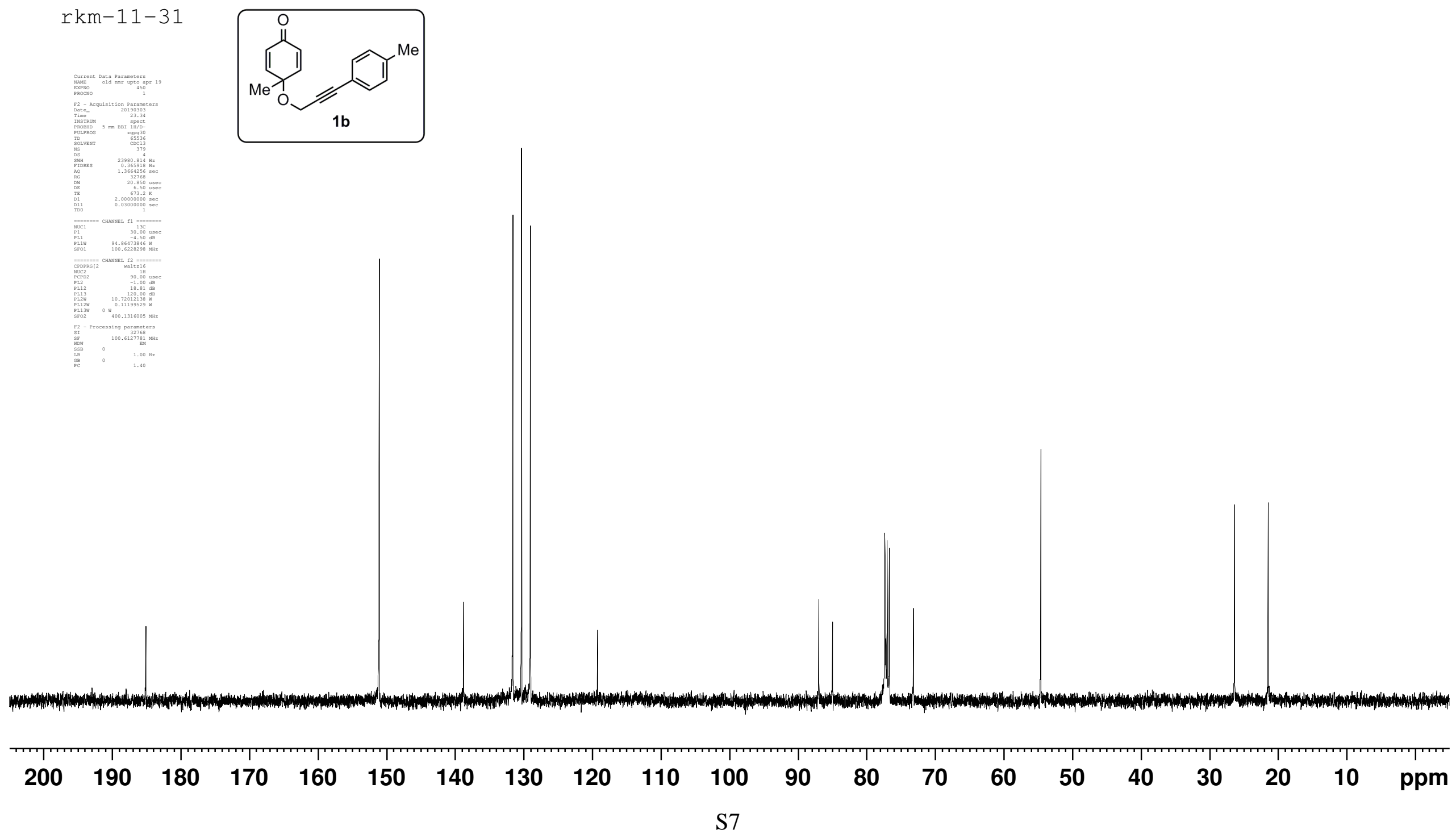



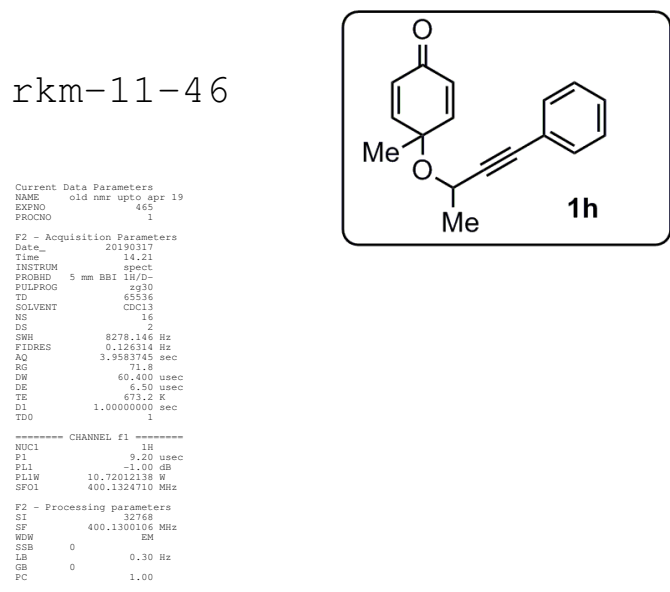

$1 \mathrm{~h}$

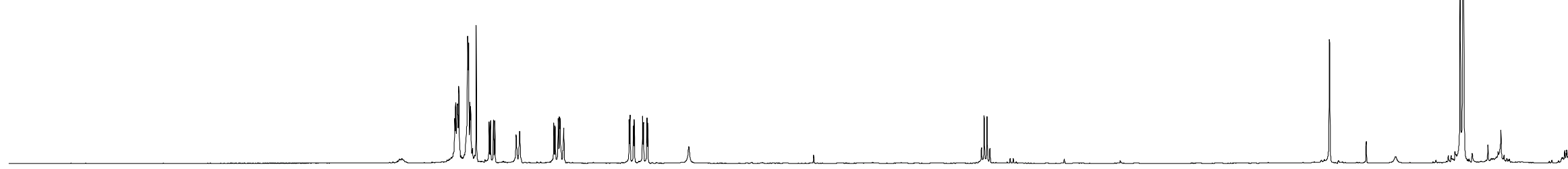

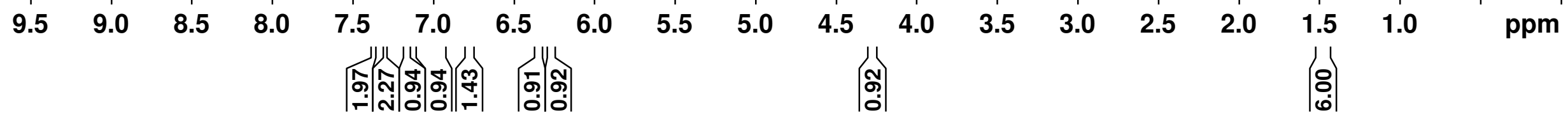



1

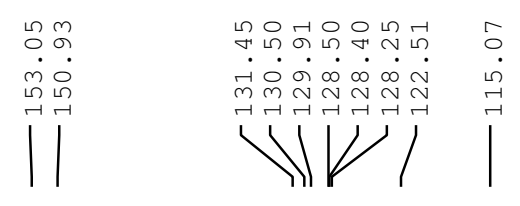

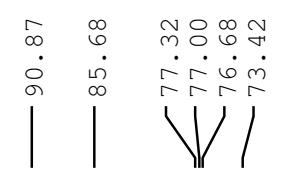
$\mid$

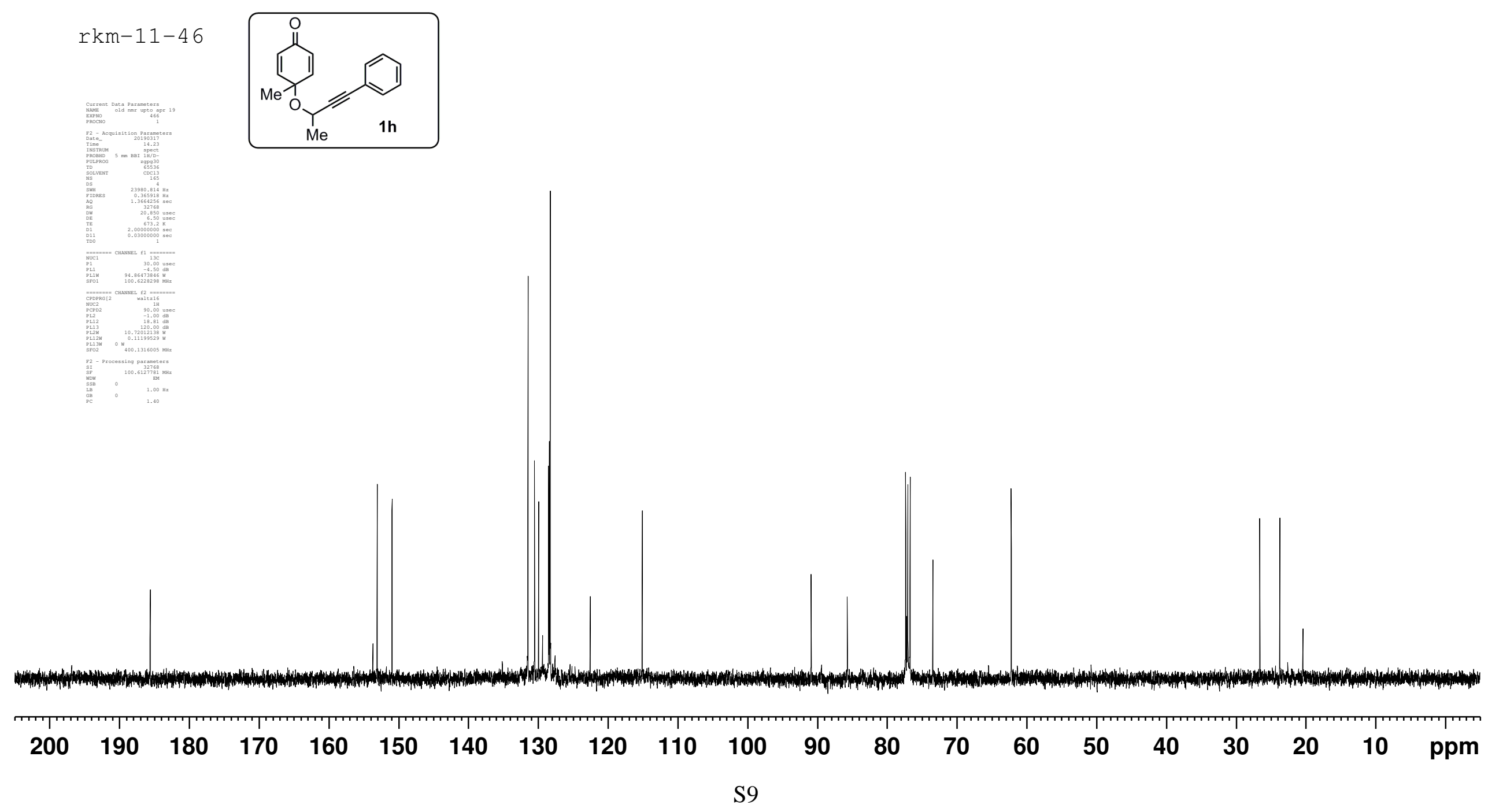



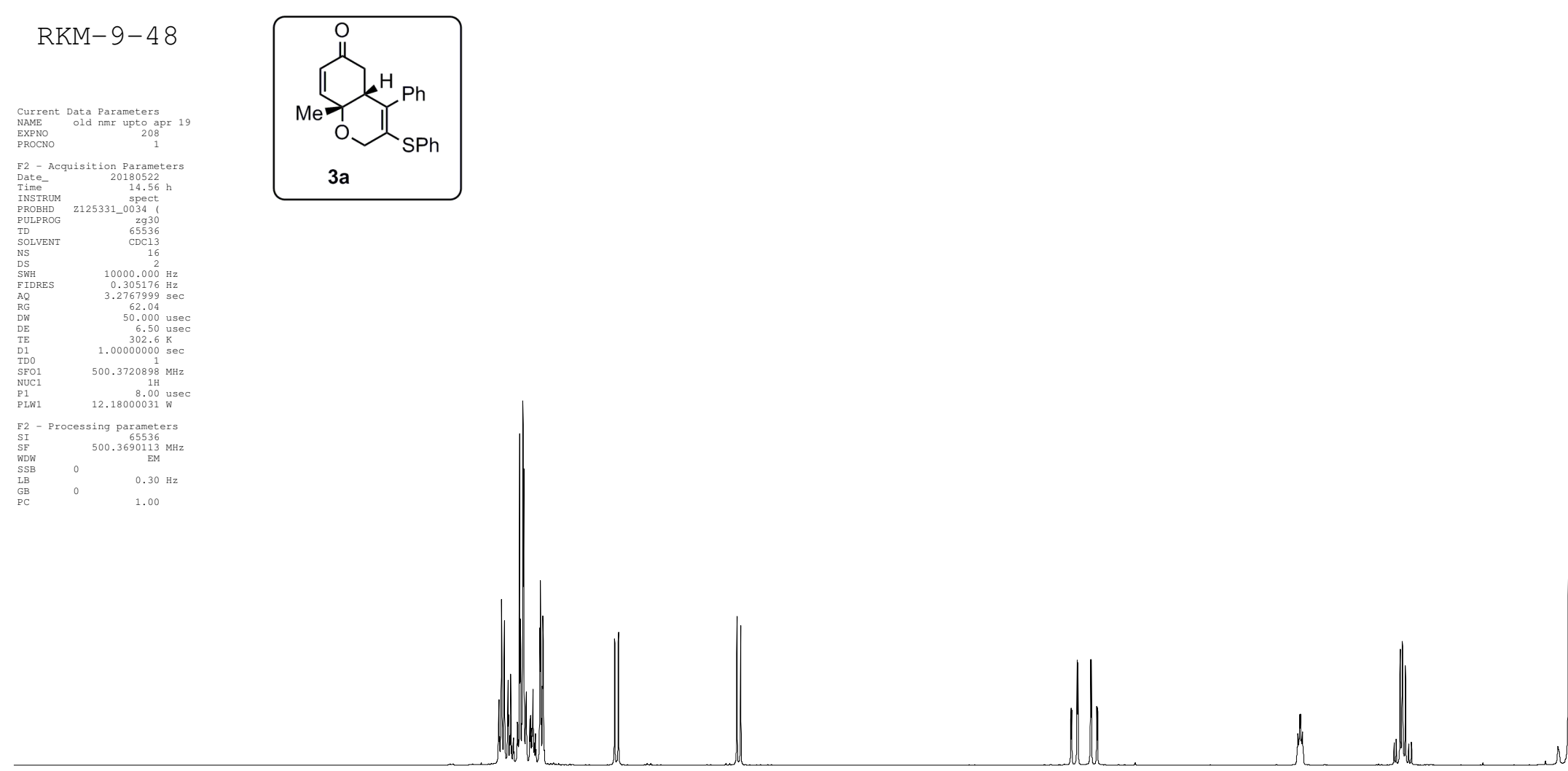

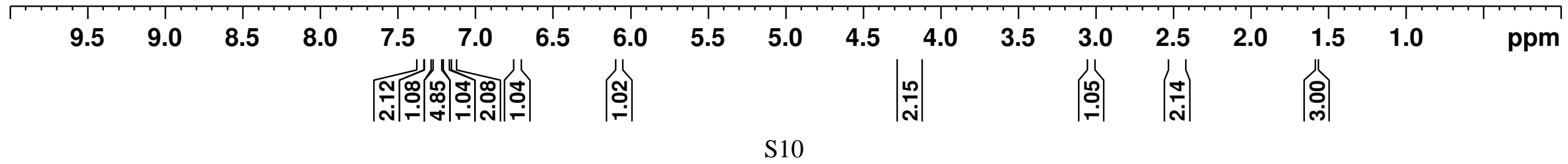



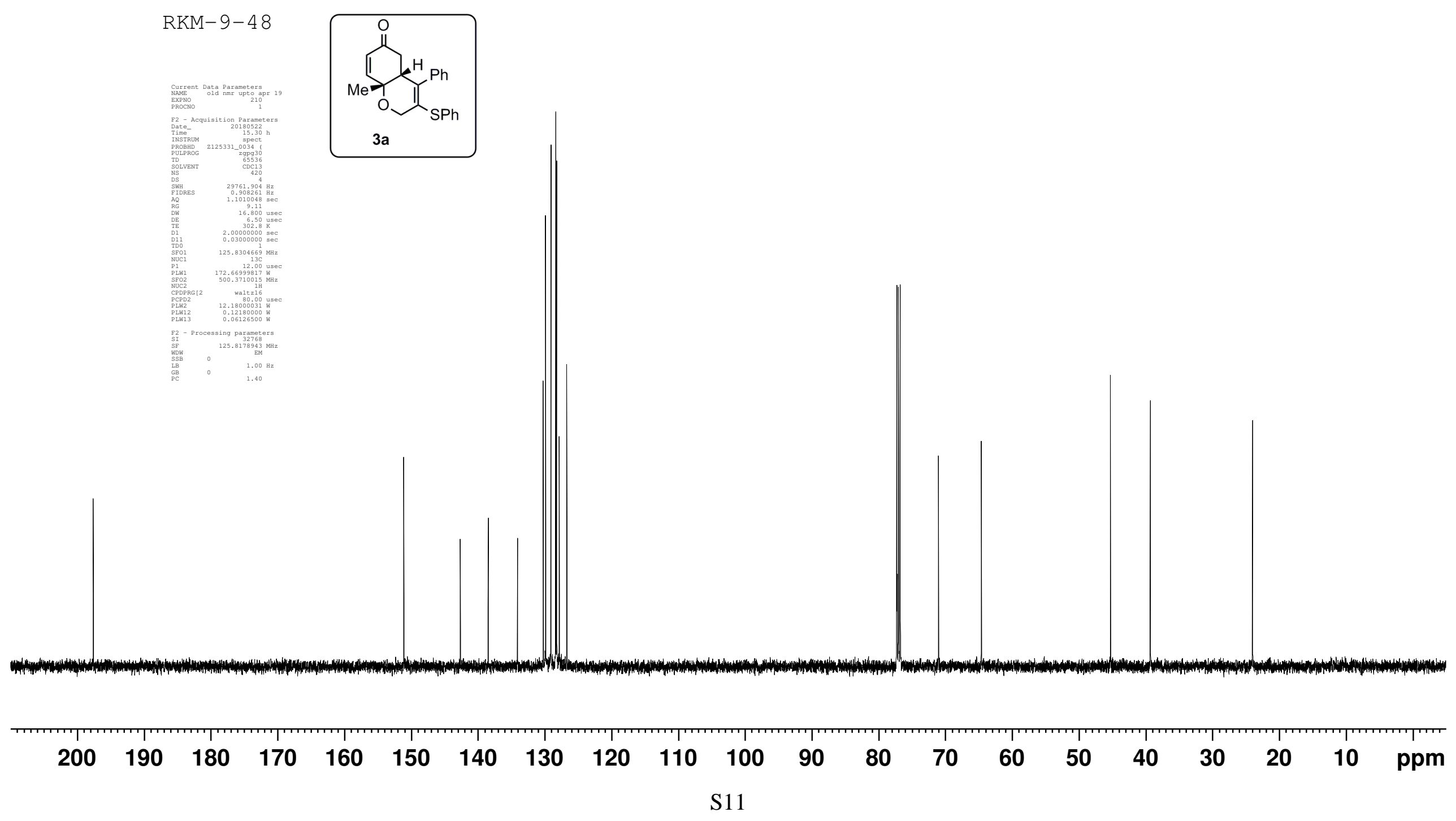


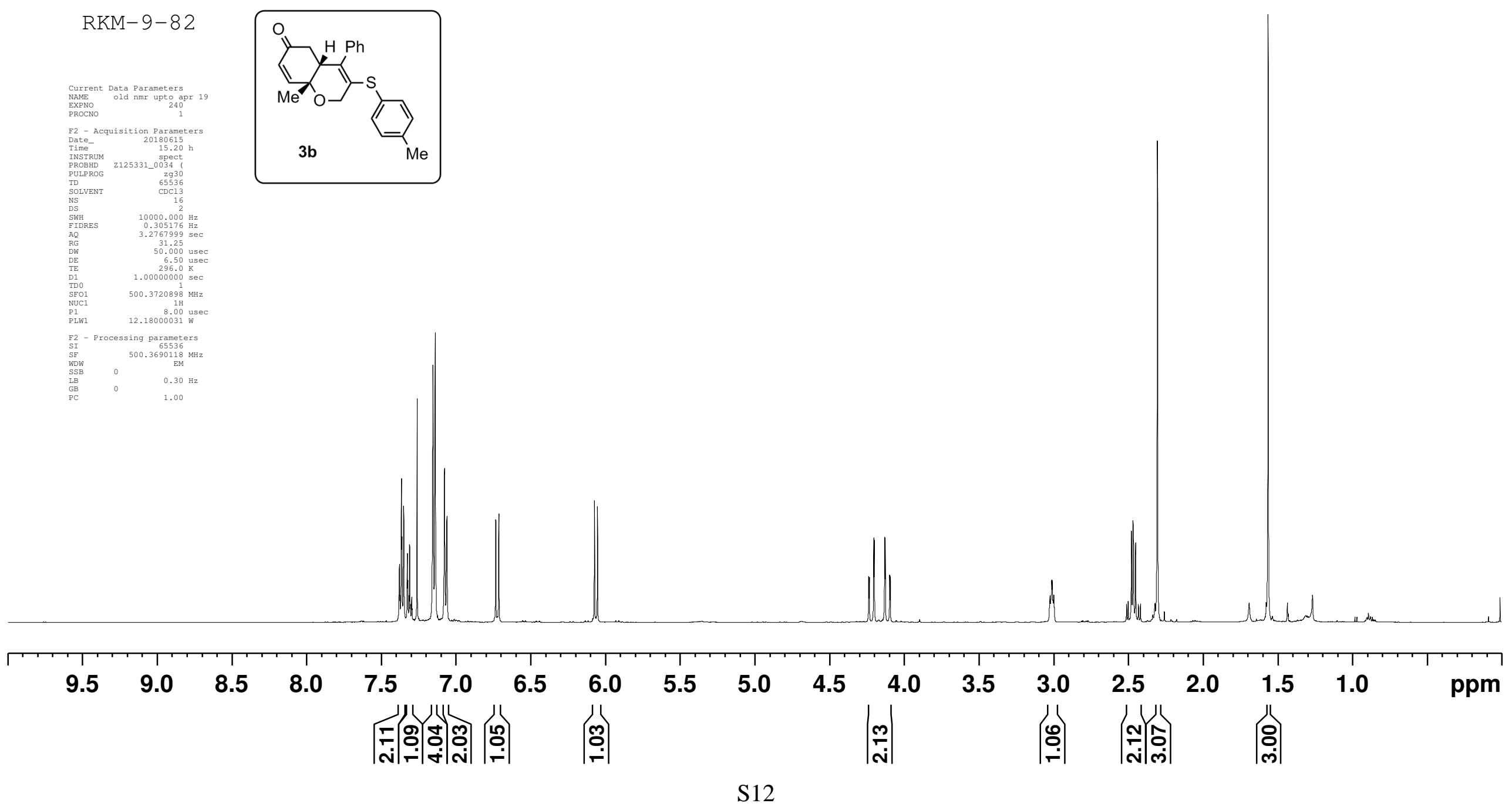



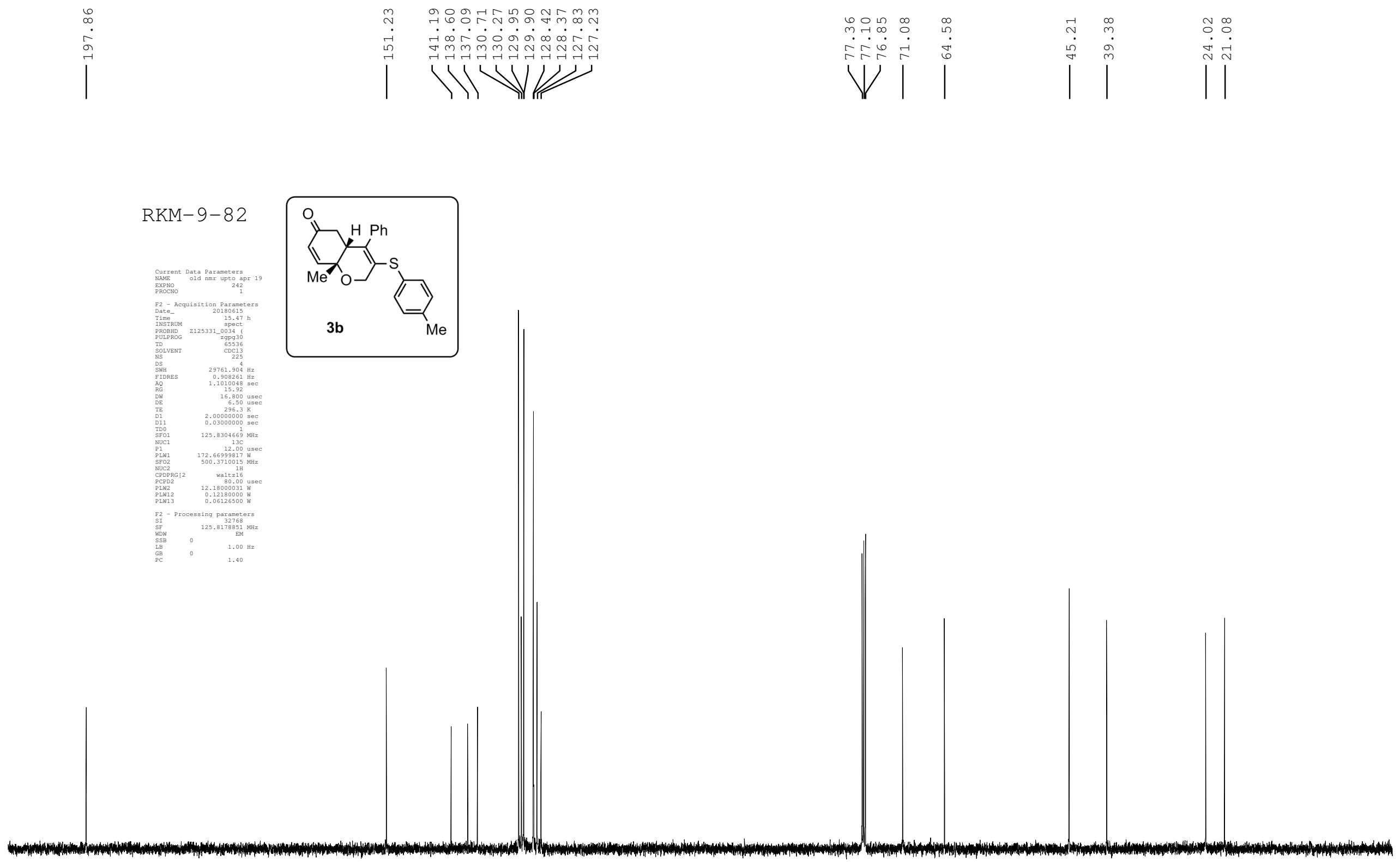


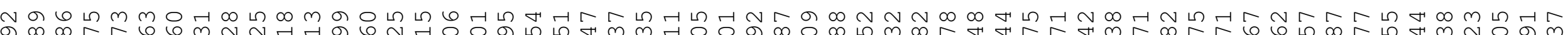

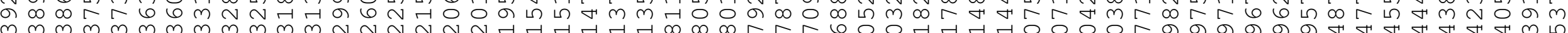

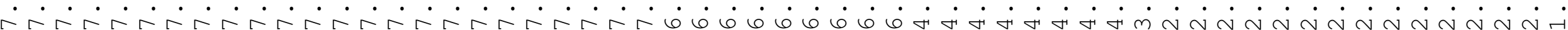

$\longrightarrow$

inim

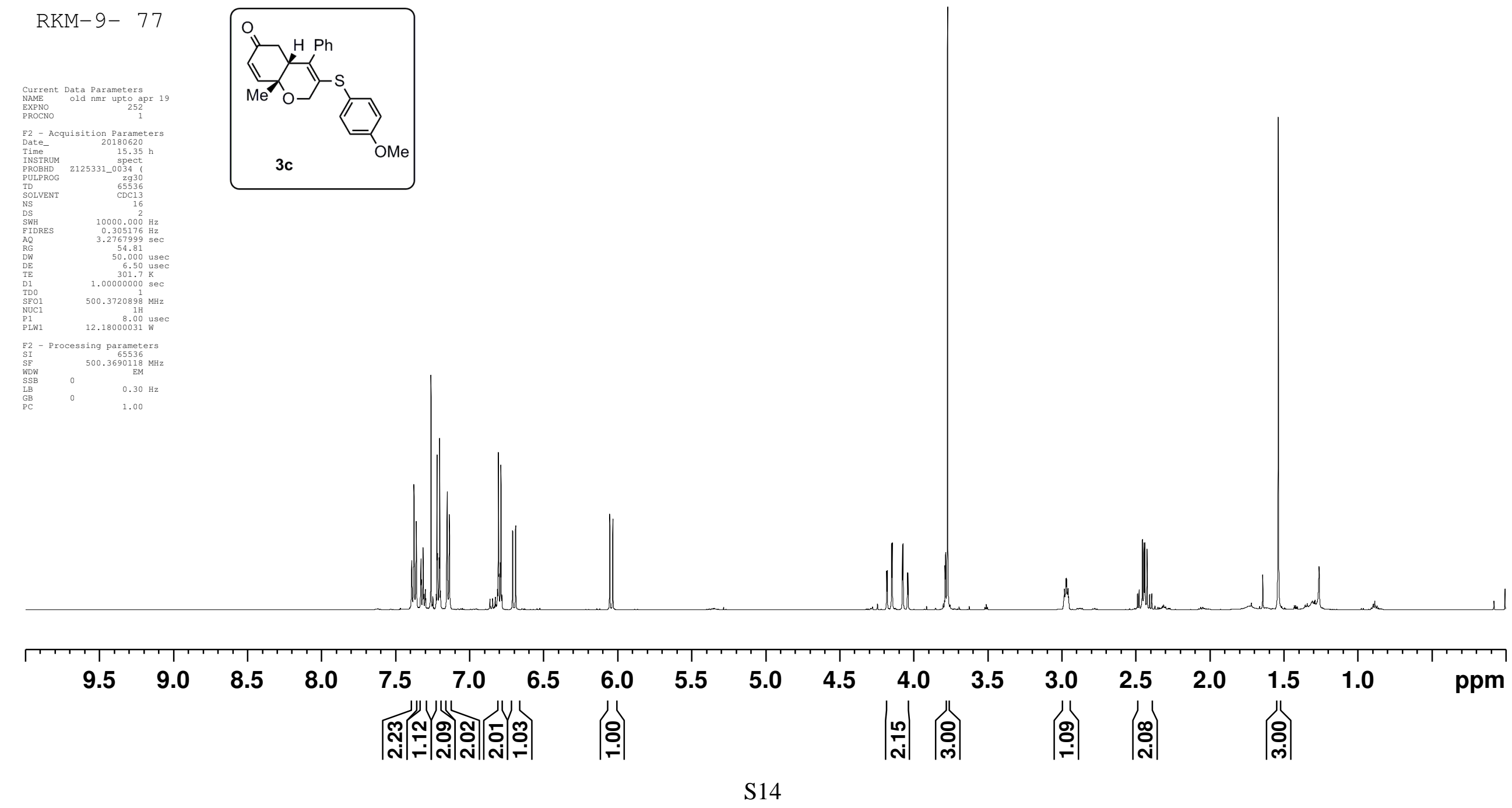

IIIII 


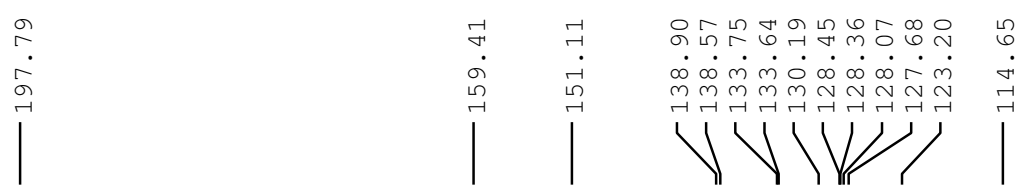

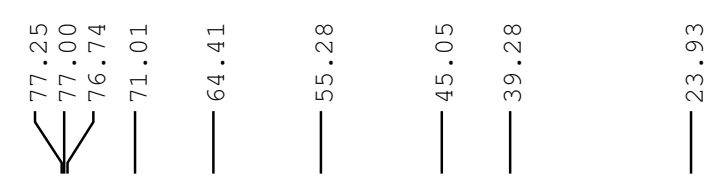

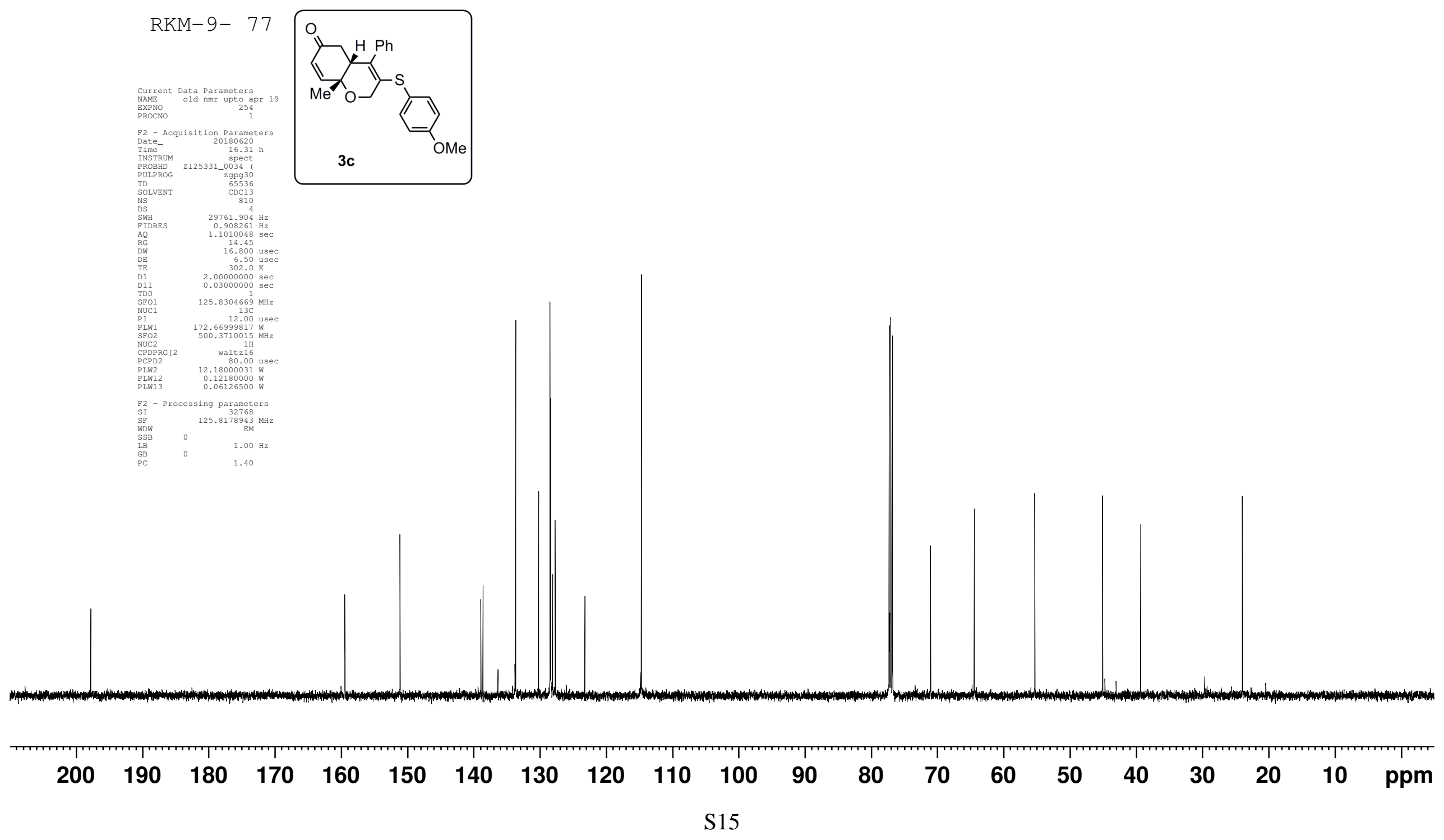



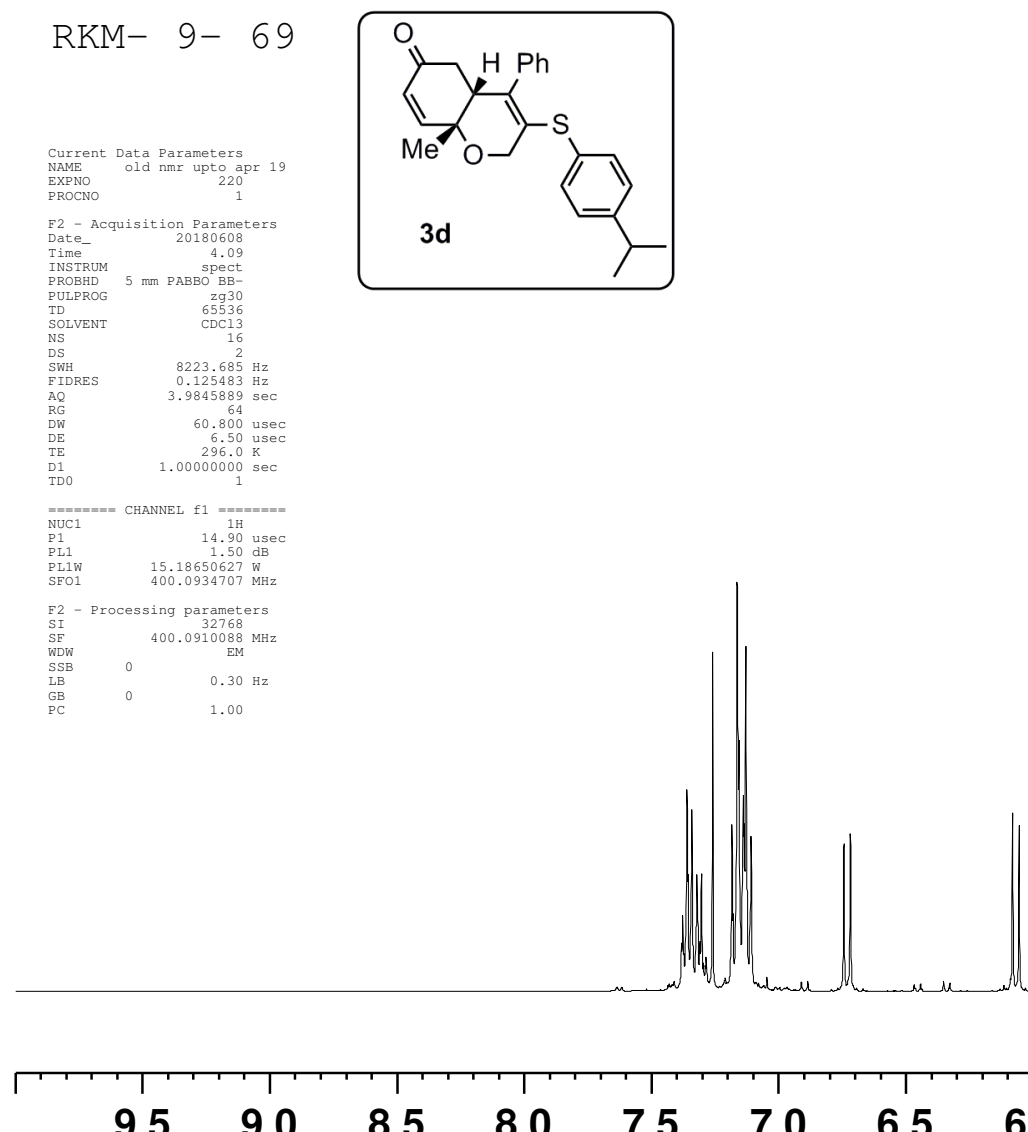

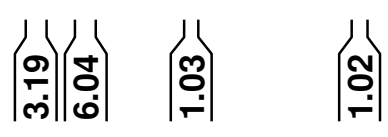

ชิ-0.0

5.5

5.0

4.5

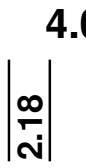

$|\stackrel{\infty}{\mathrm{N}}|^{4.0}$

3.5

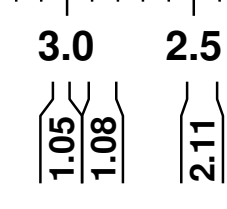

2.52 .0

1.51 .0 

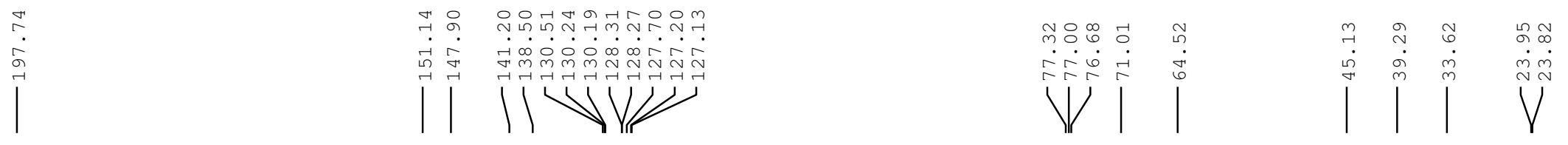

RKM- 9- 69

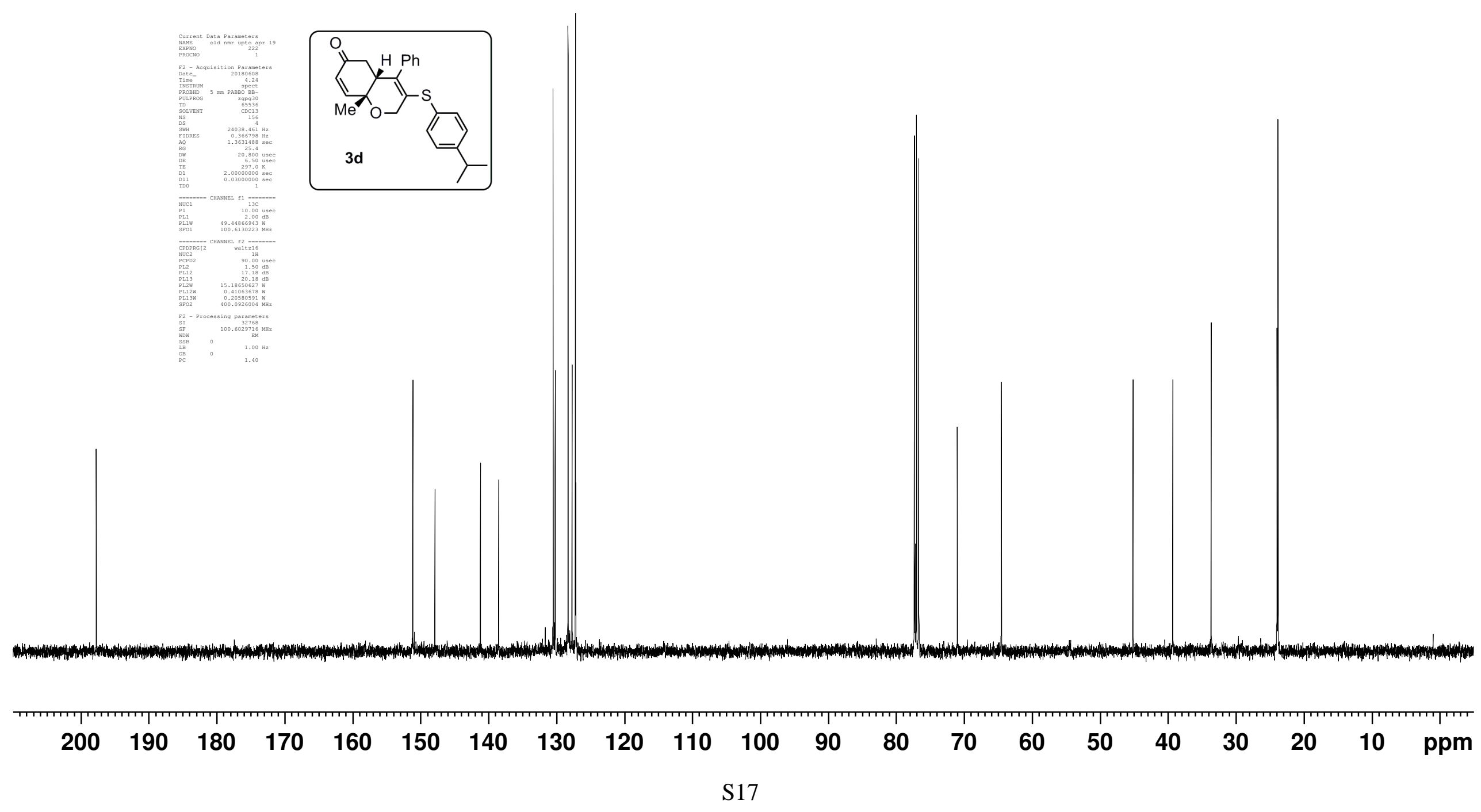




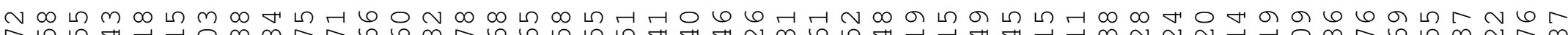
m m m m m m N N N N N H

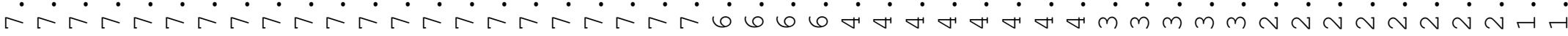

$\longrightarrow$
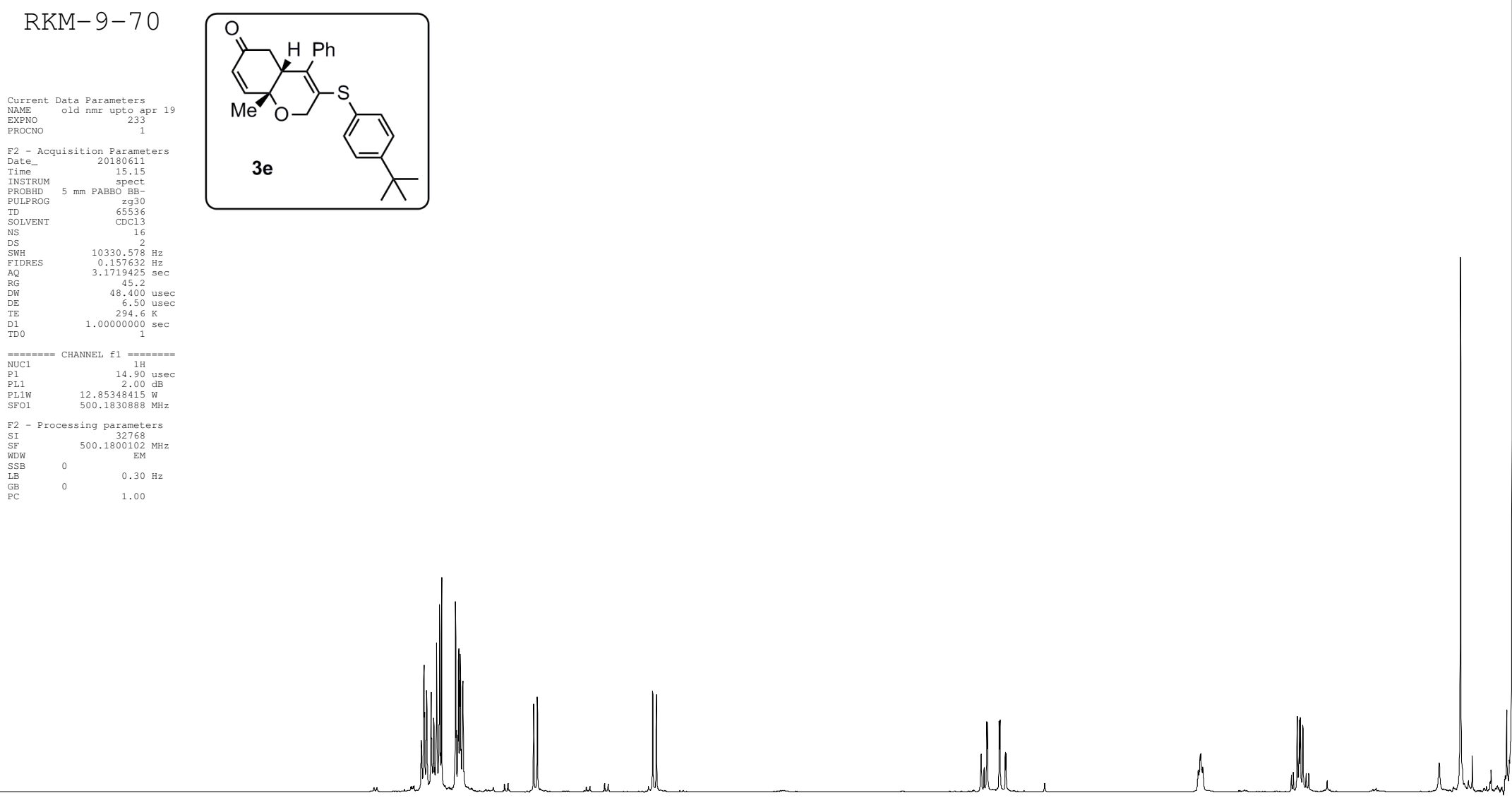

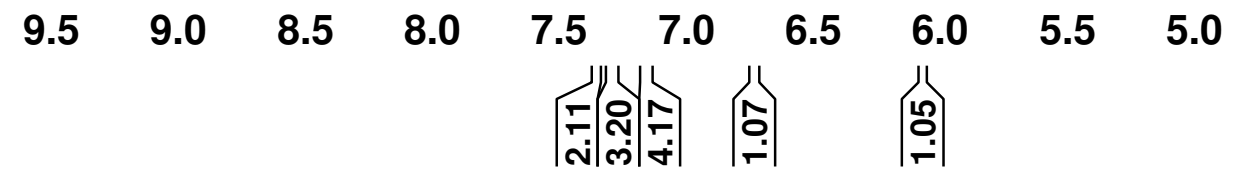

$4.5 \quad 4.0$

$\left|\begin{array}{l}\stackrel{\infty}{N} \\ \text { N̦}\end{array}\right|$

3.5
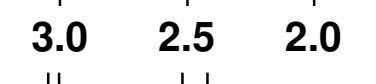

|

1.51 .0

各家| 


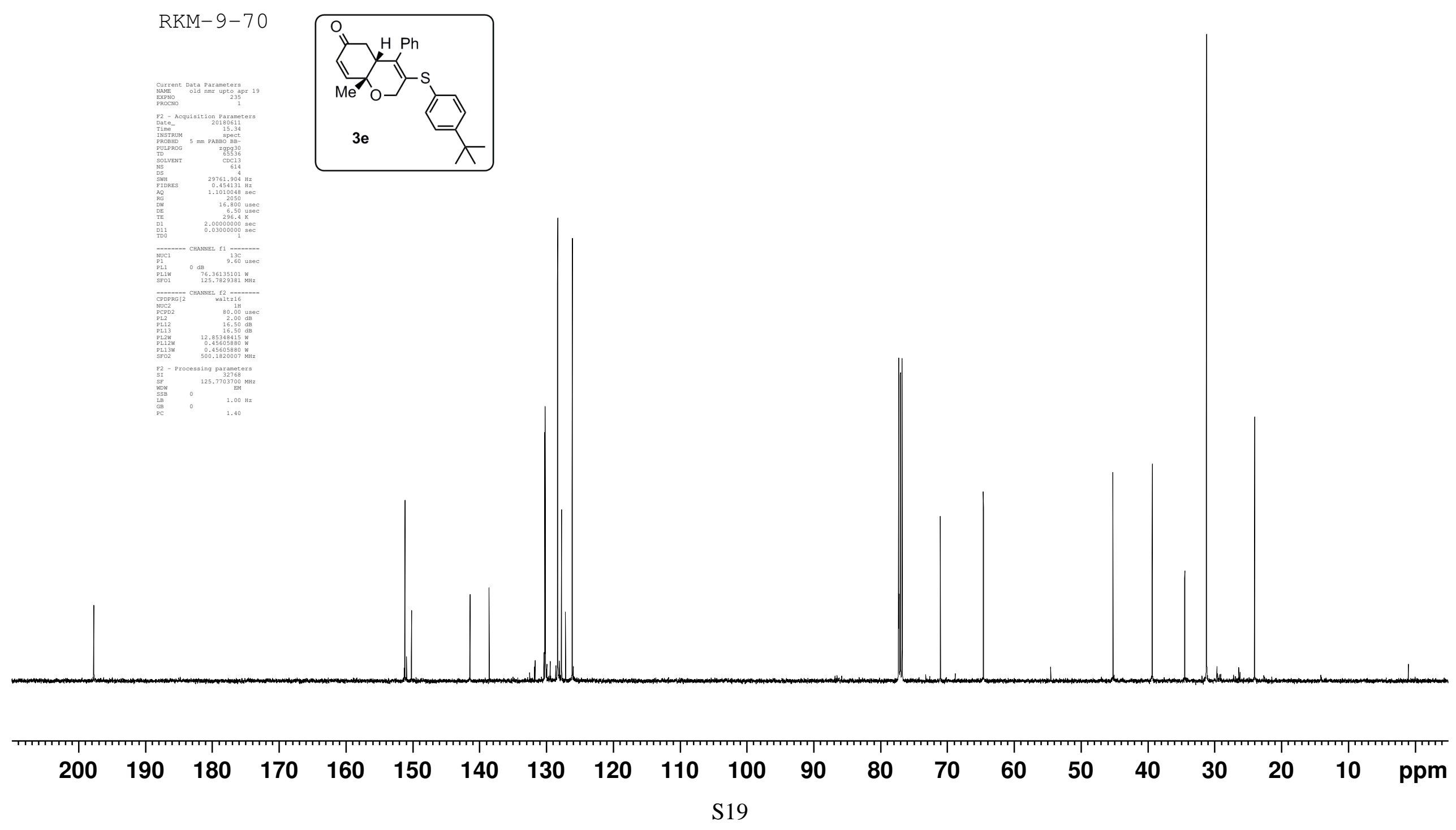




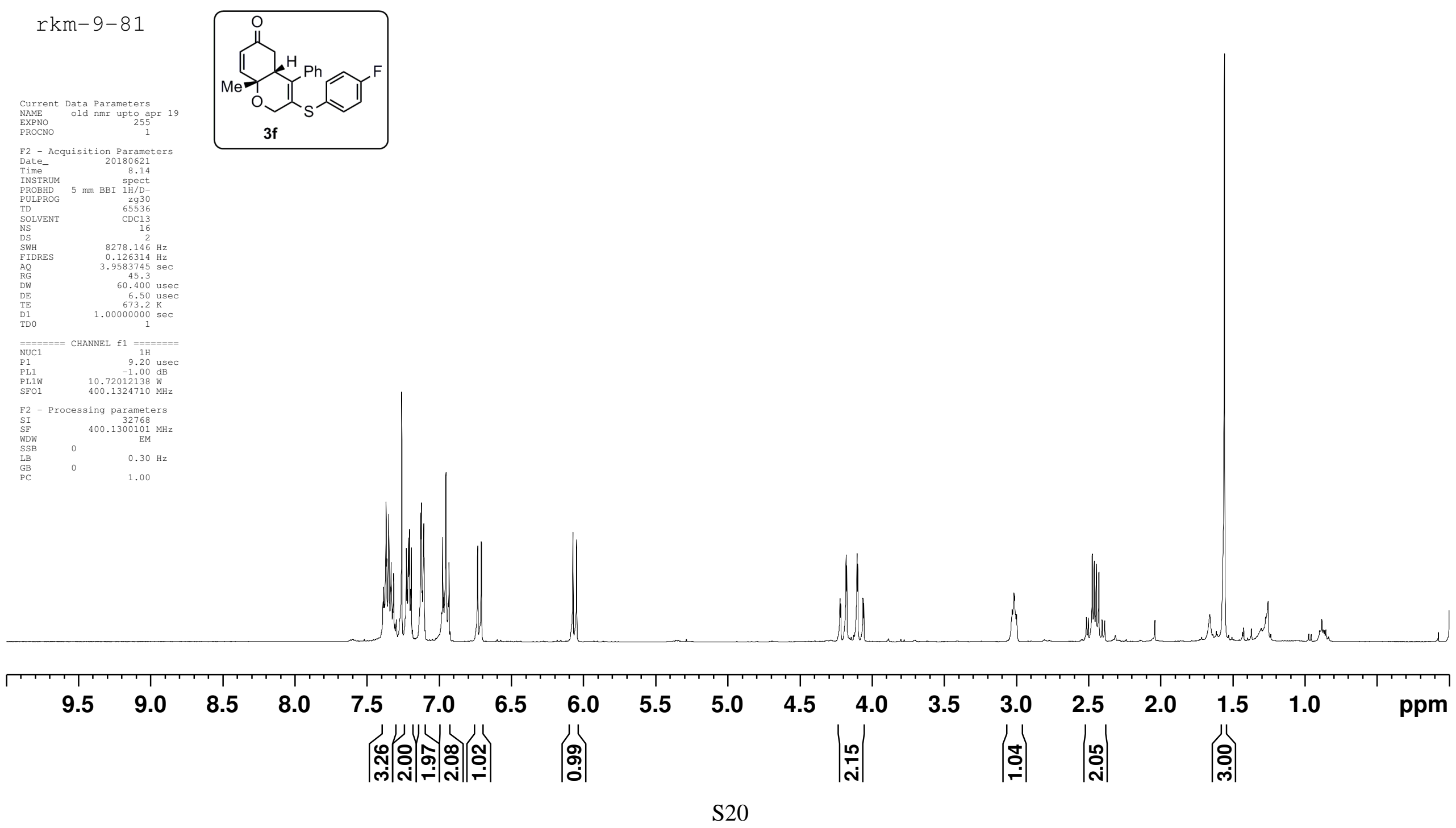




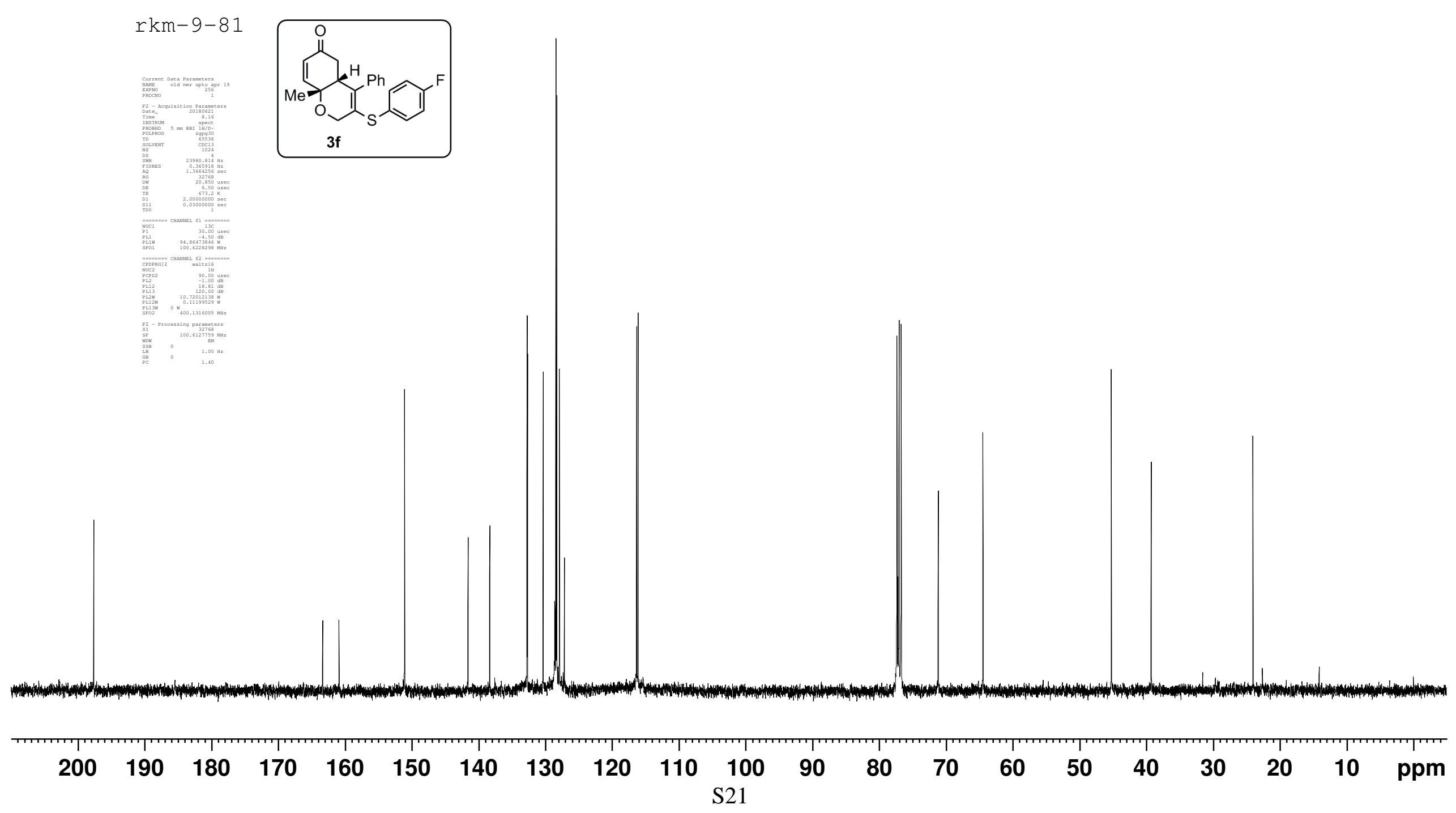




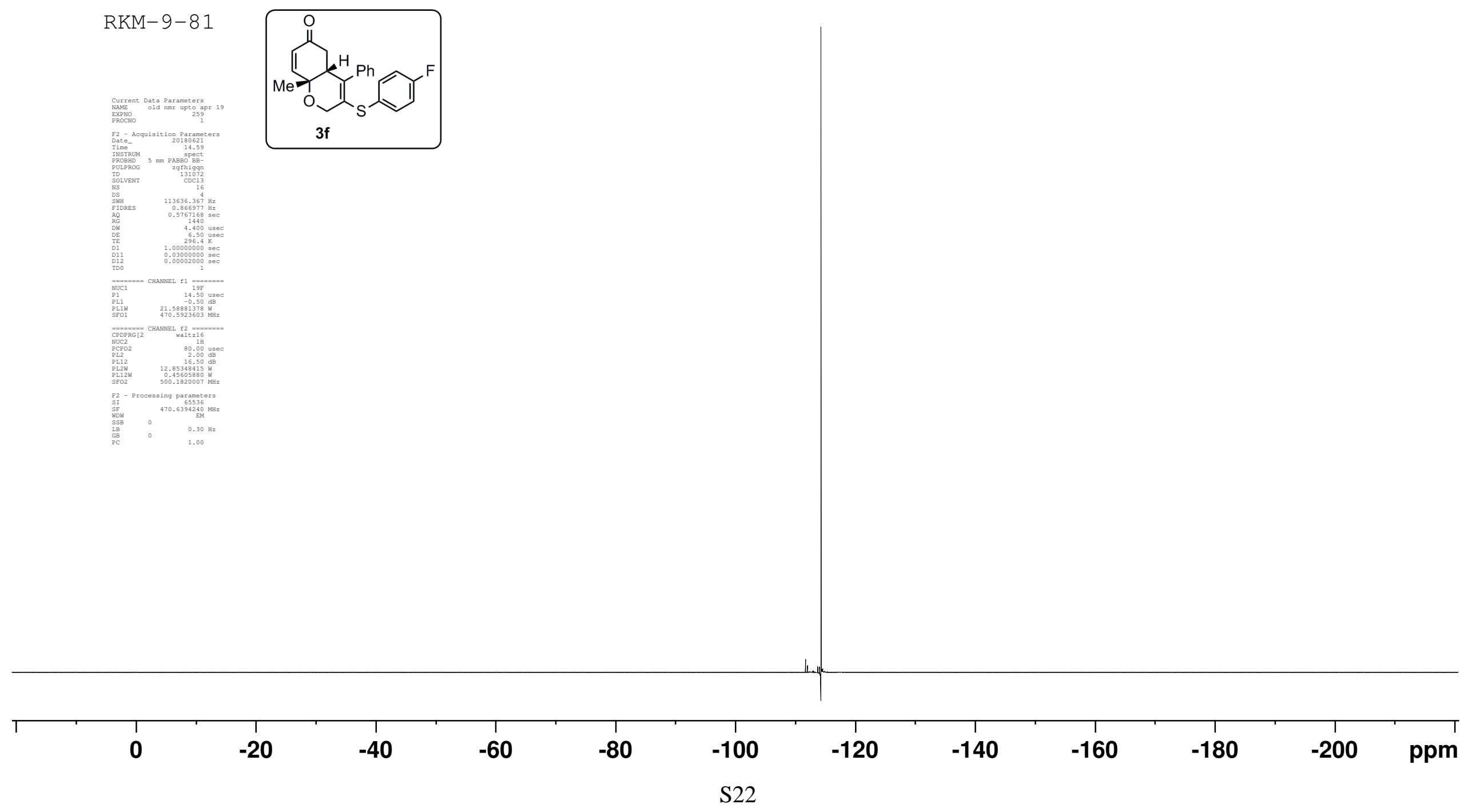






$r k m-9-80$

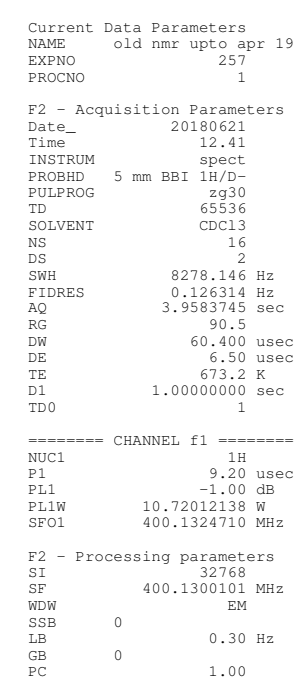

$3 g$
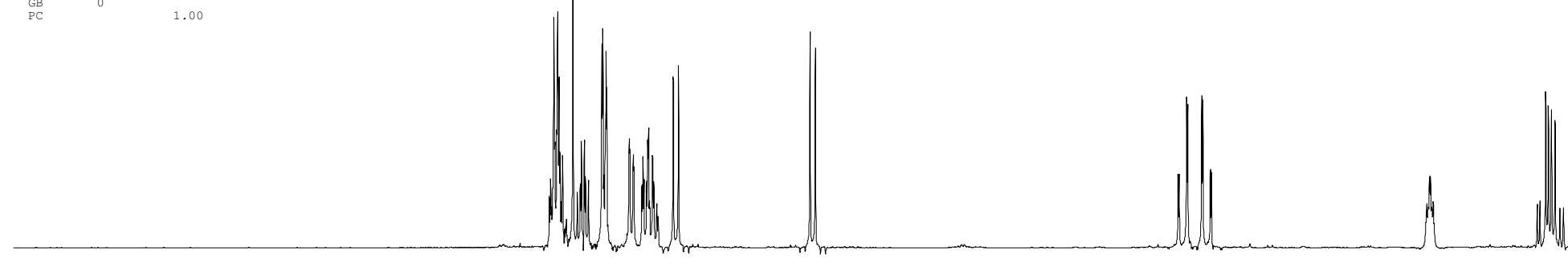

N

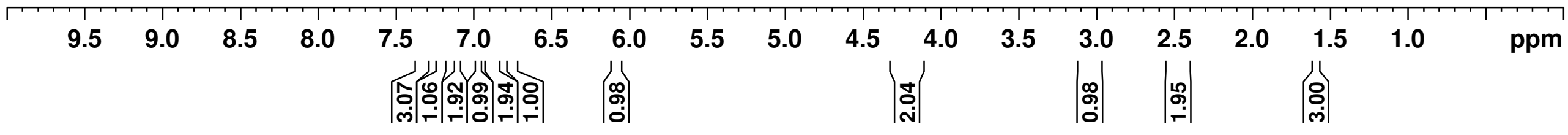



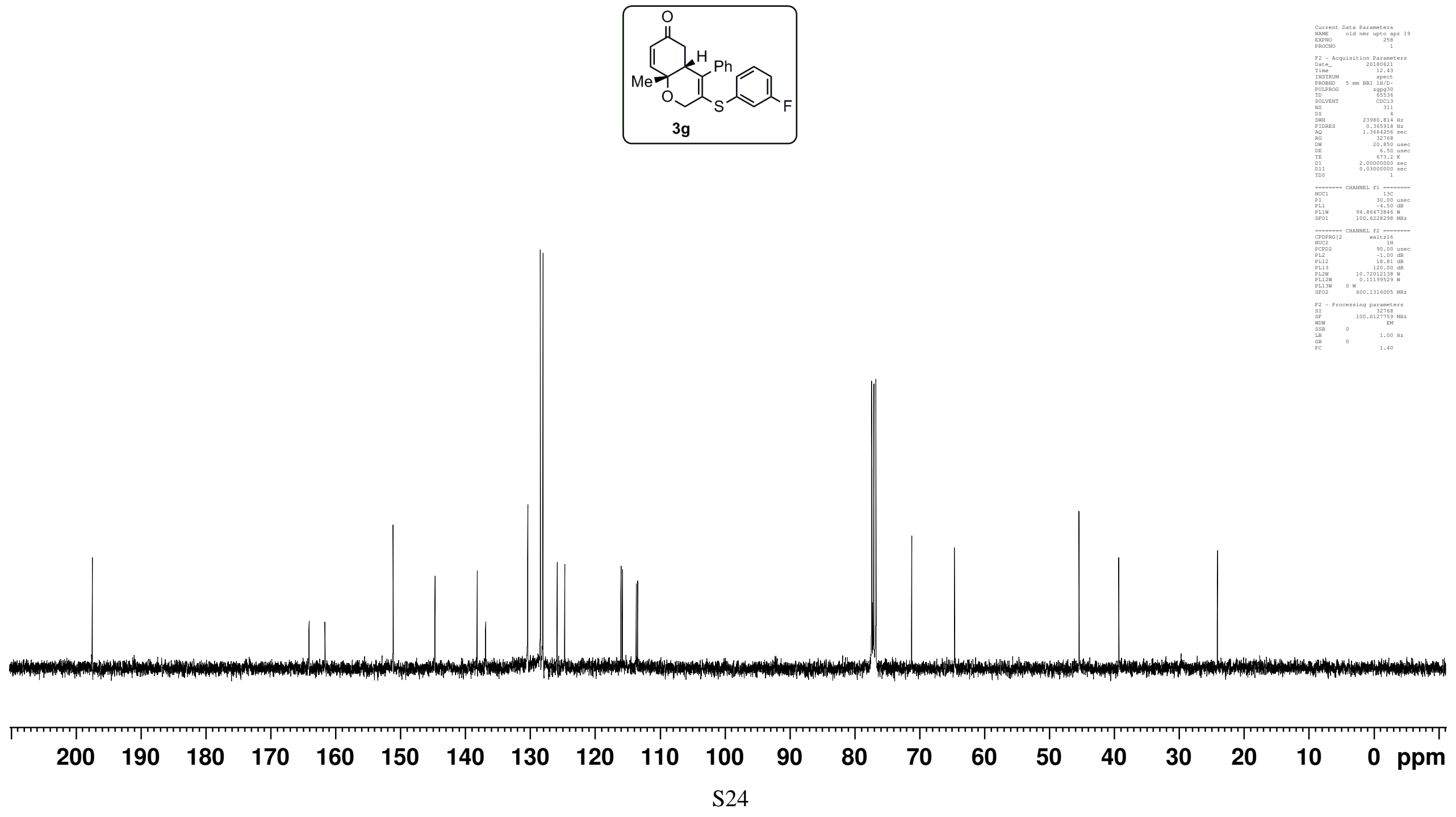
RKM-9-80

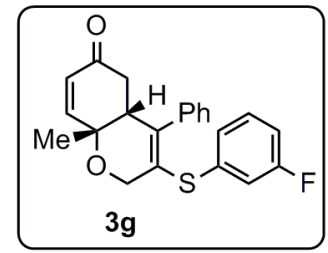

\begin{tabular}{|c|c|c|c|c|c|c|c|c|c|c|c|}
\hline 1 & $T$ & $T$ & $T$ & $T$ & $T$ & $T$ & $T$ & $T$ & $T$ & T & \\
\hline 0 & -20 & -40 & -60 & -80 & -100 & -120 & -140 & -160 & -180 & -200 & ppm \\
\hline
\end{tabular}


IIIII

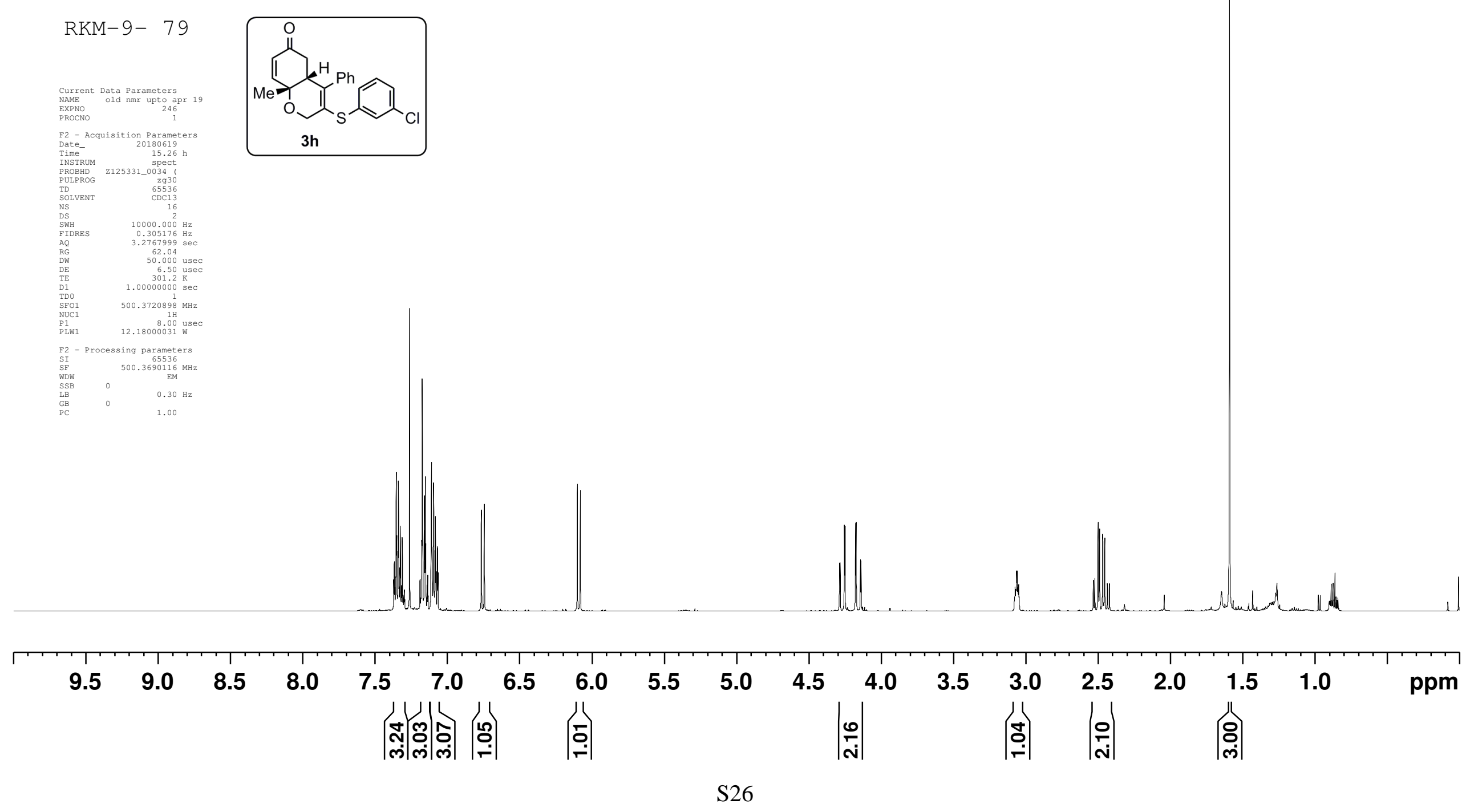




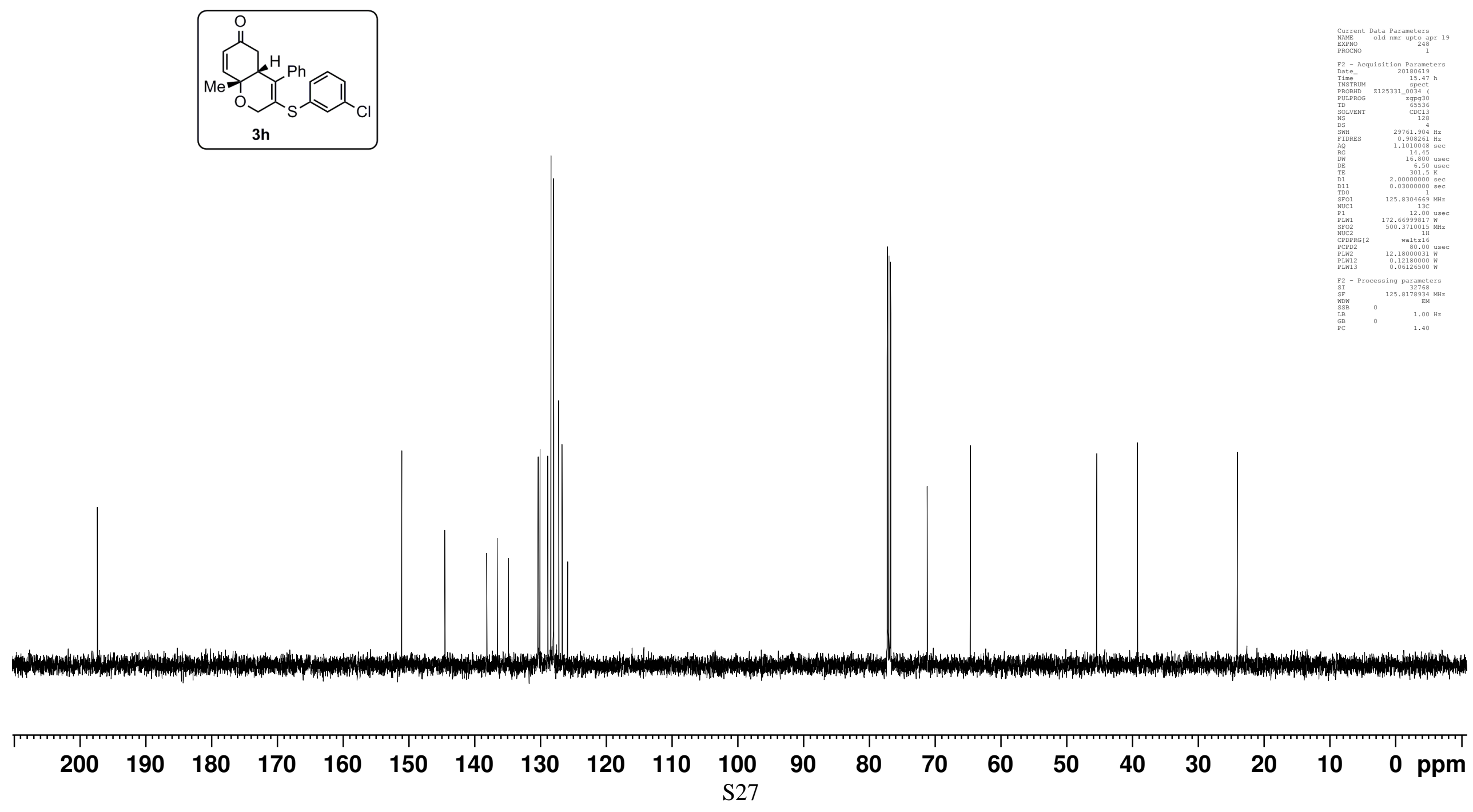




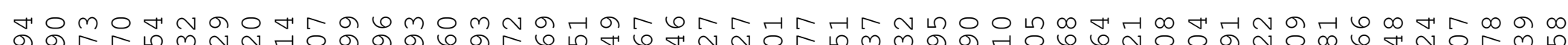
Љ)

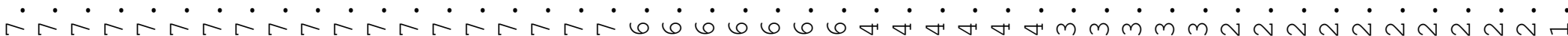
$\longrightarrow$
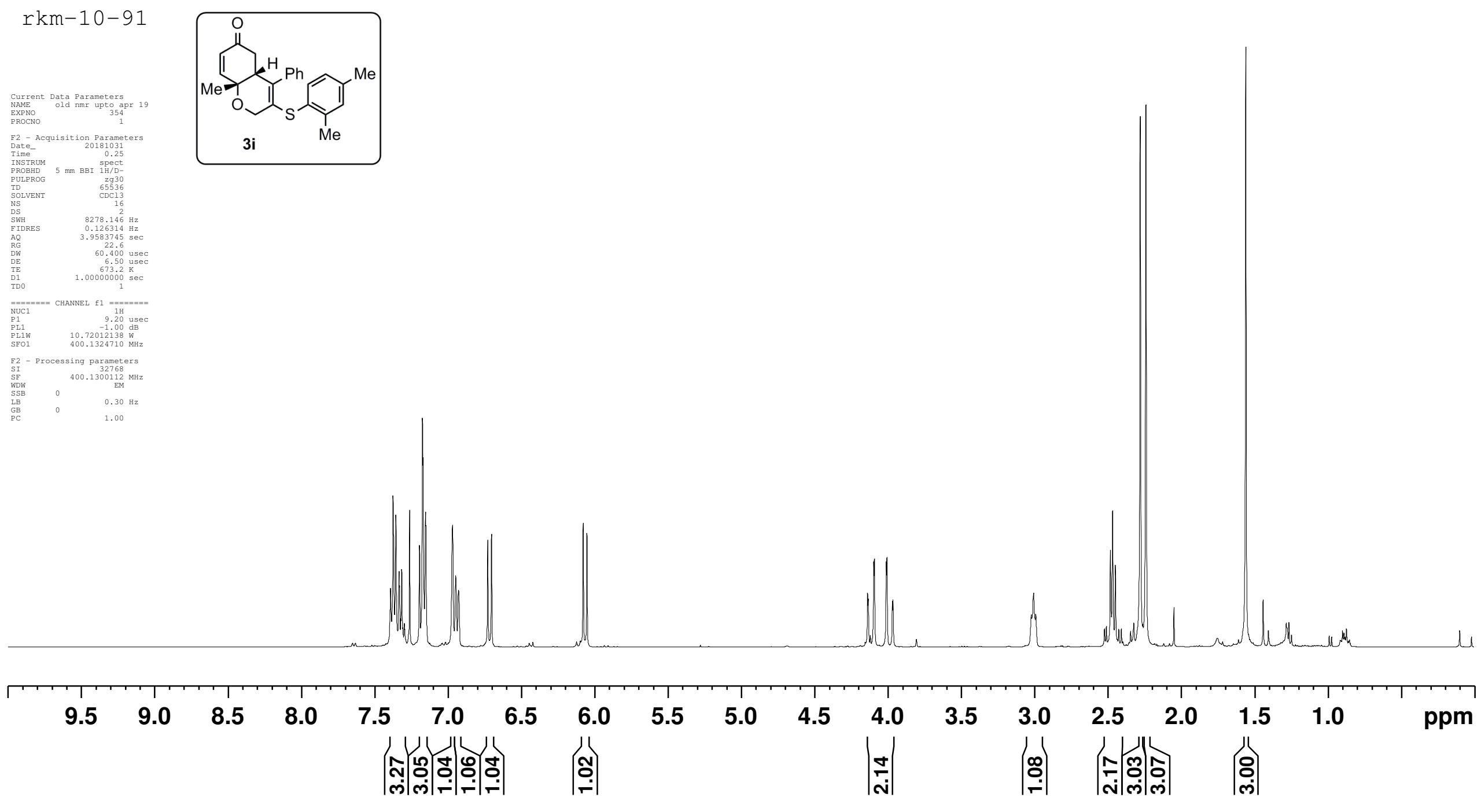

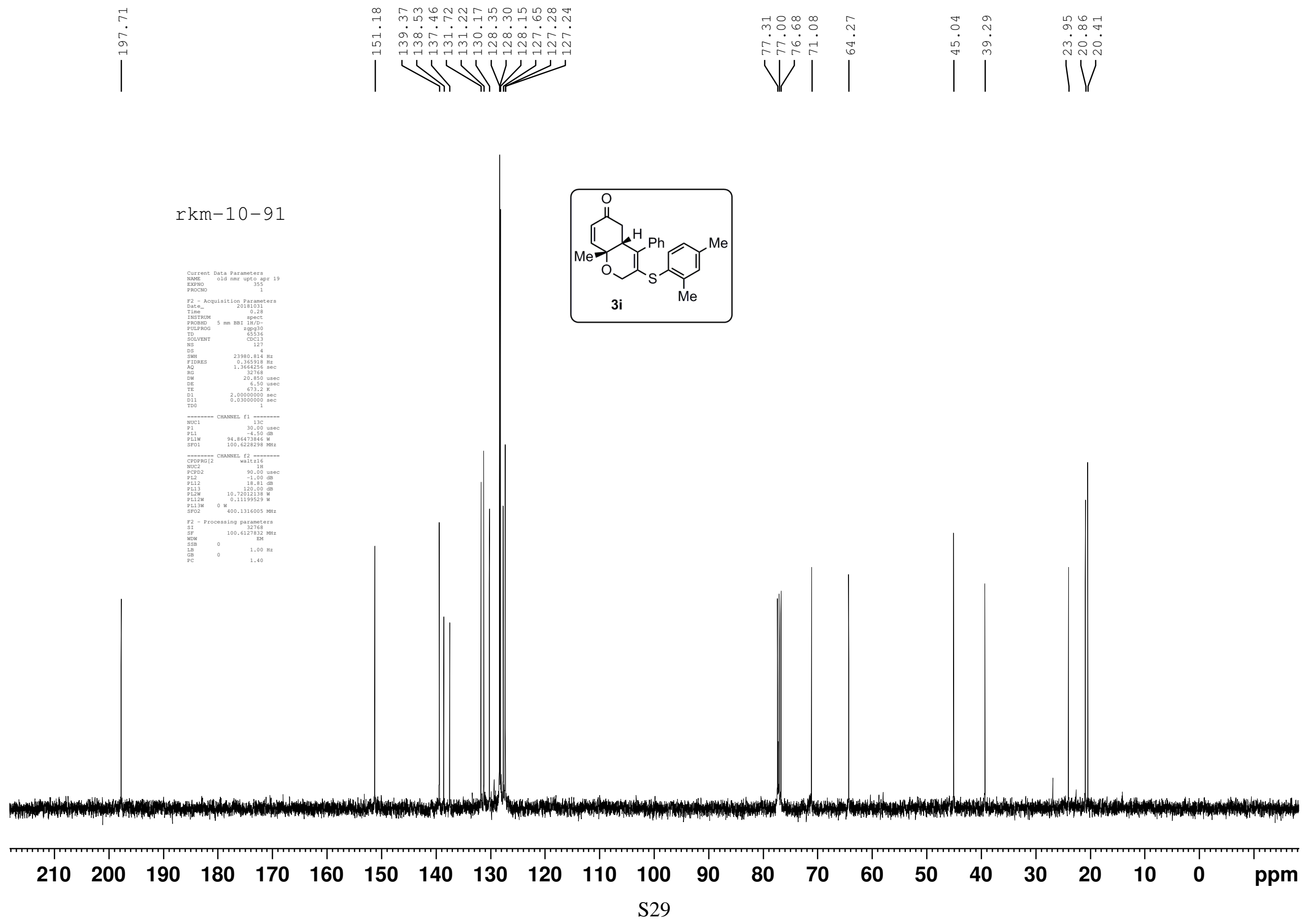


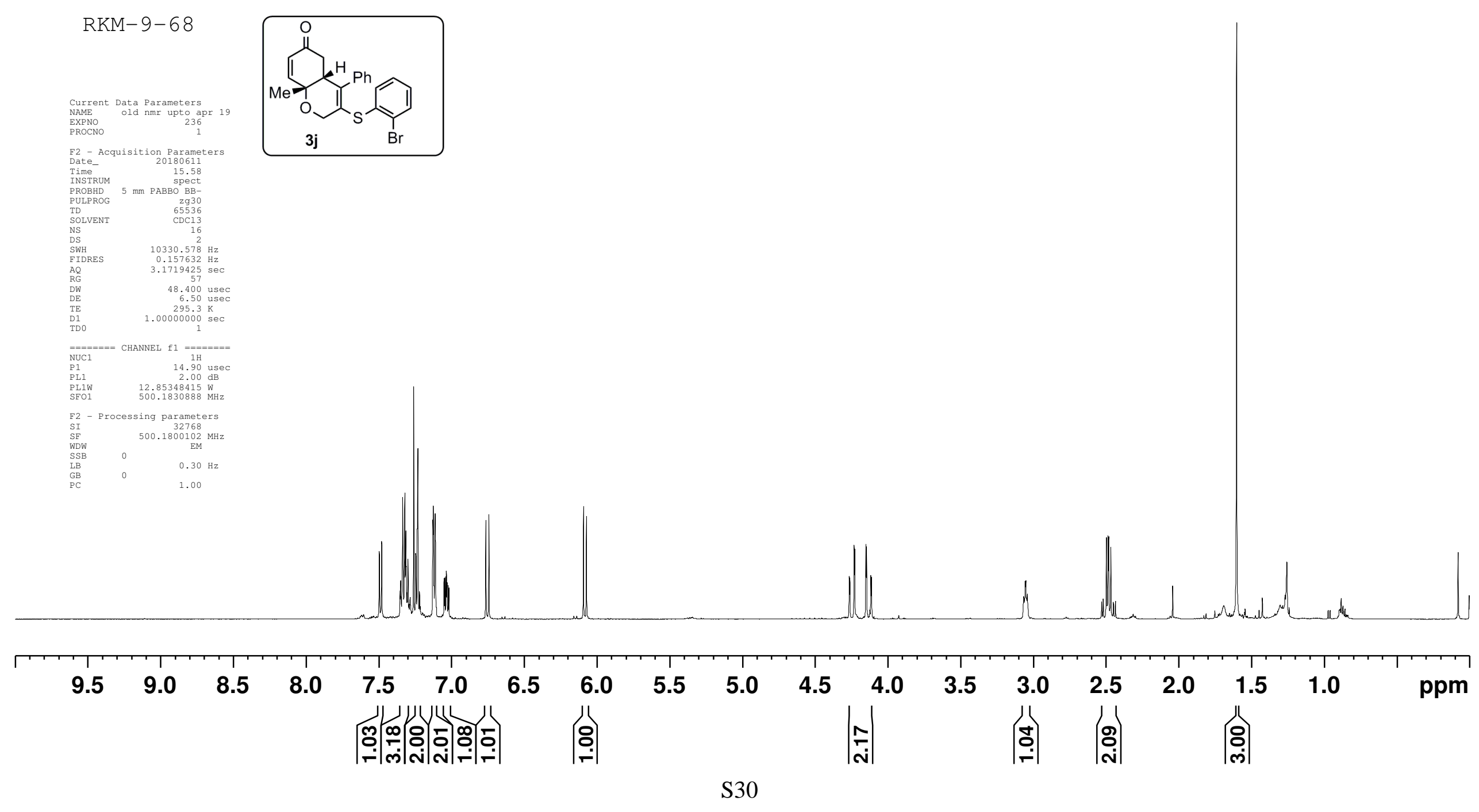




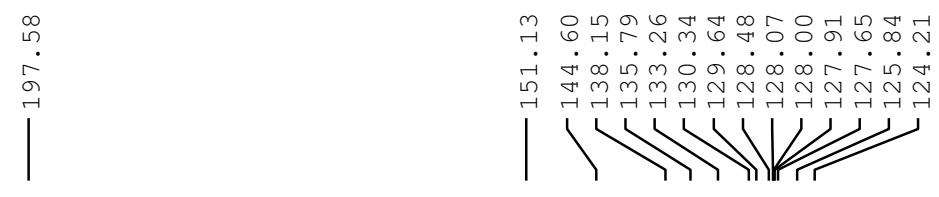

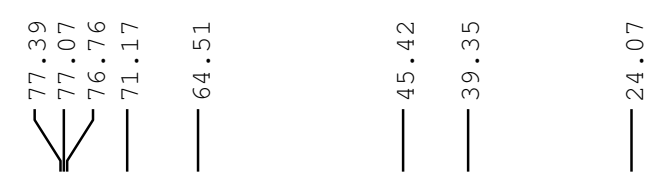

$\mathrm{RKM}-9-68$
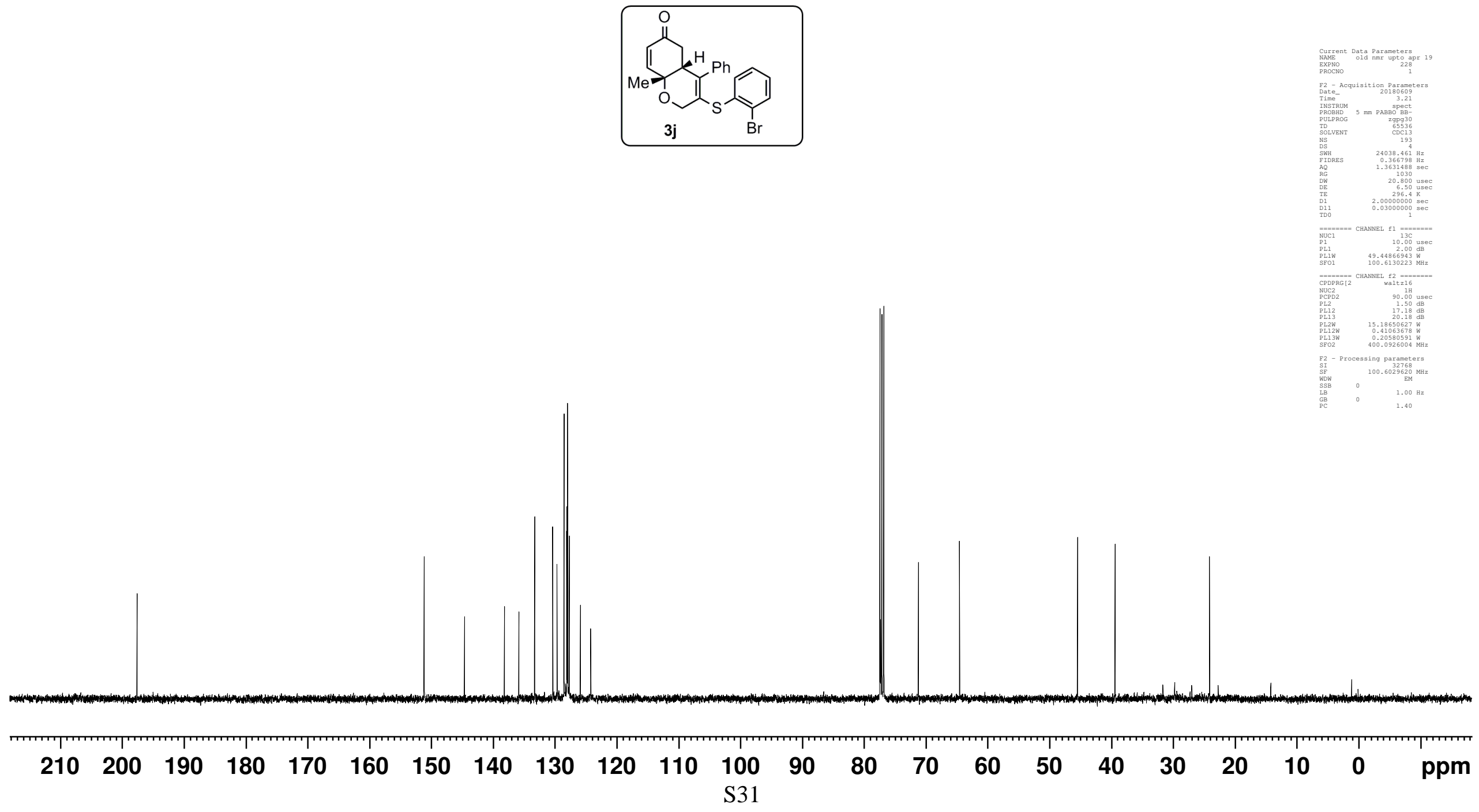
RKM- 9- 71
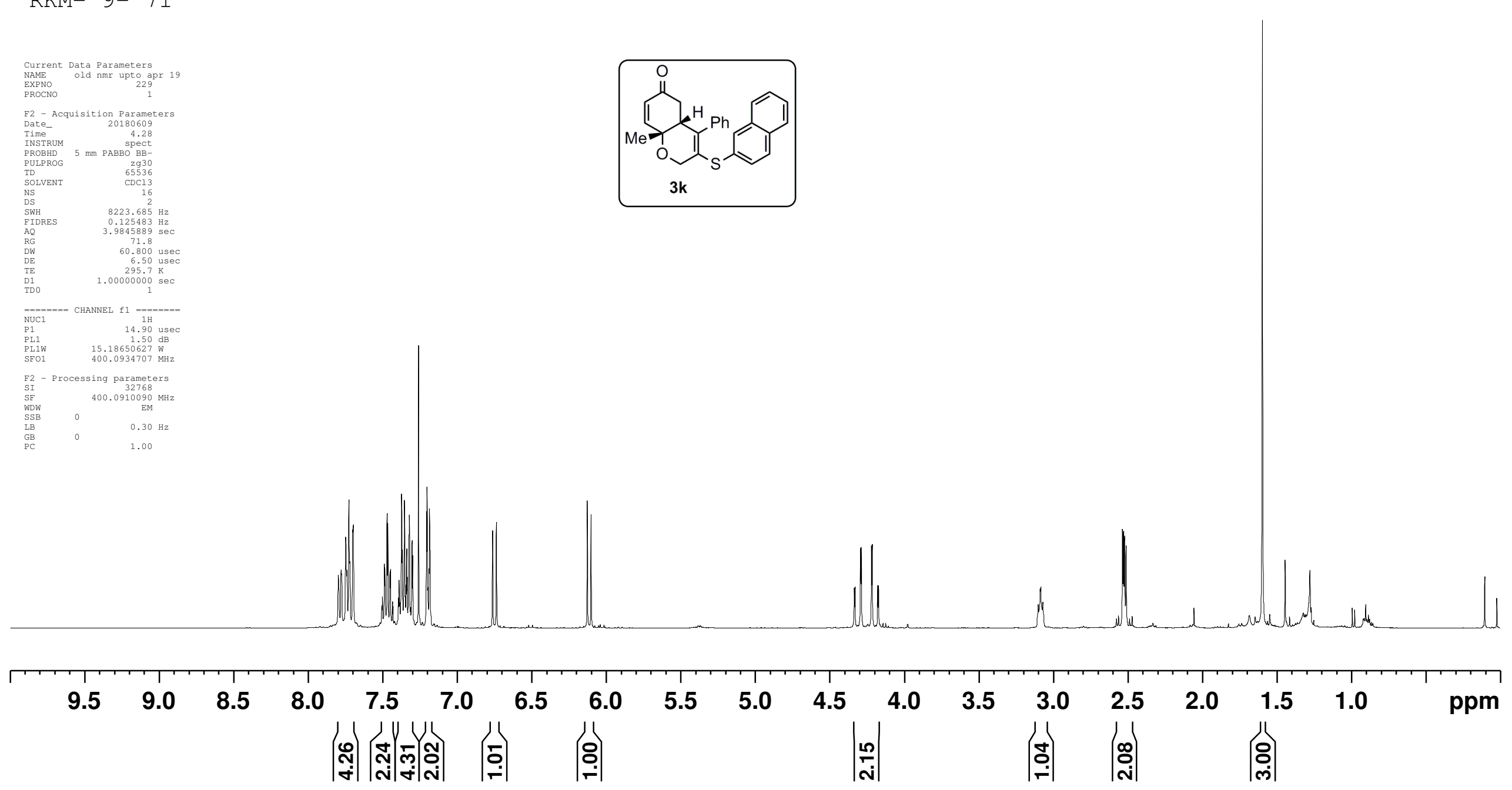

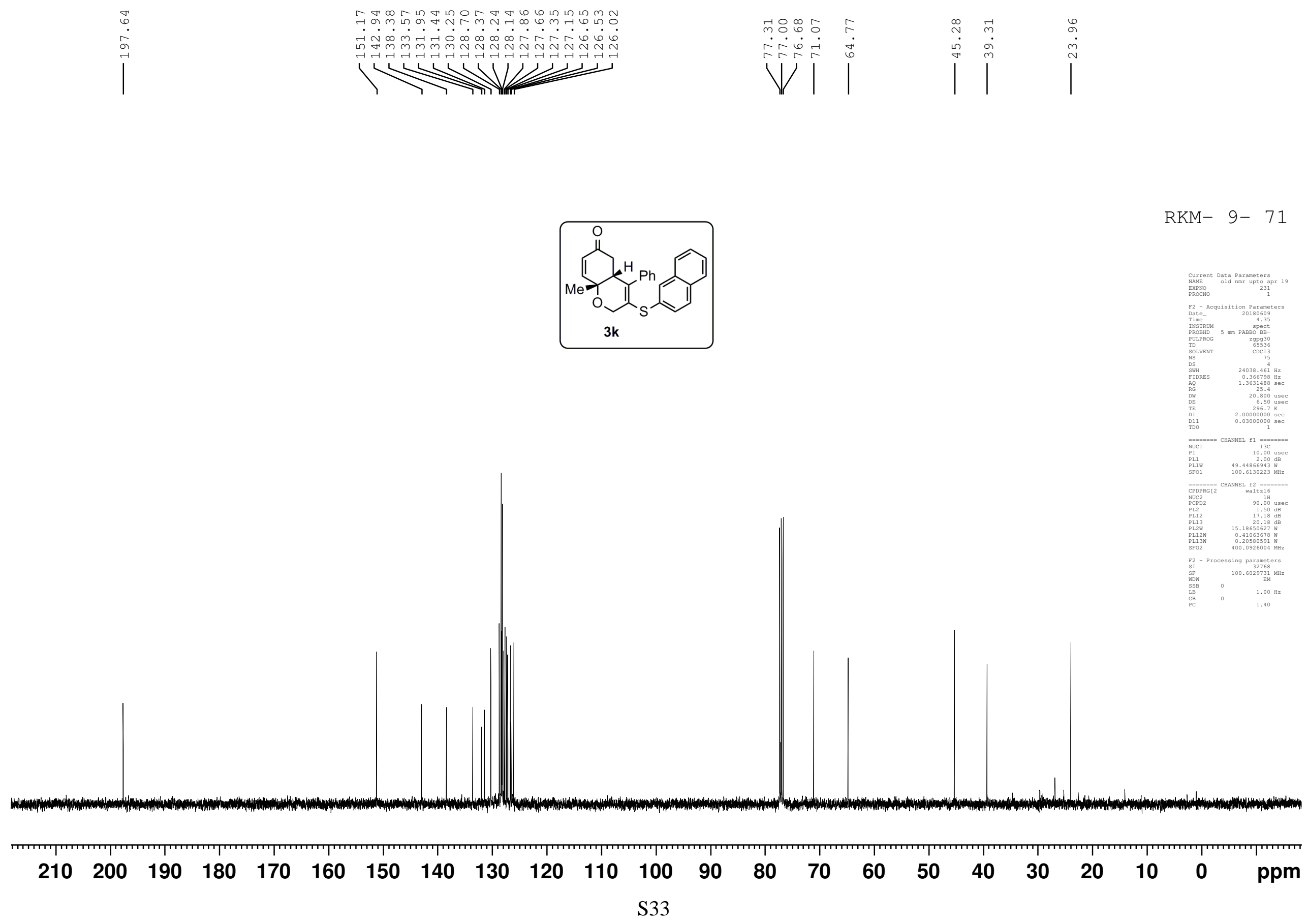

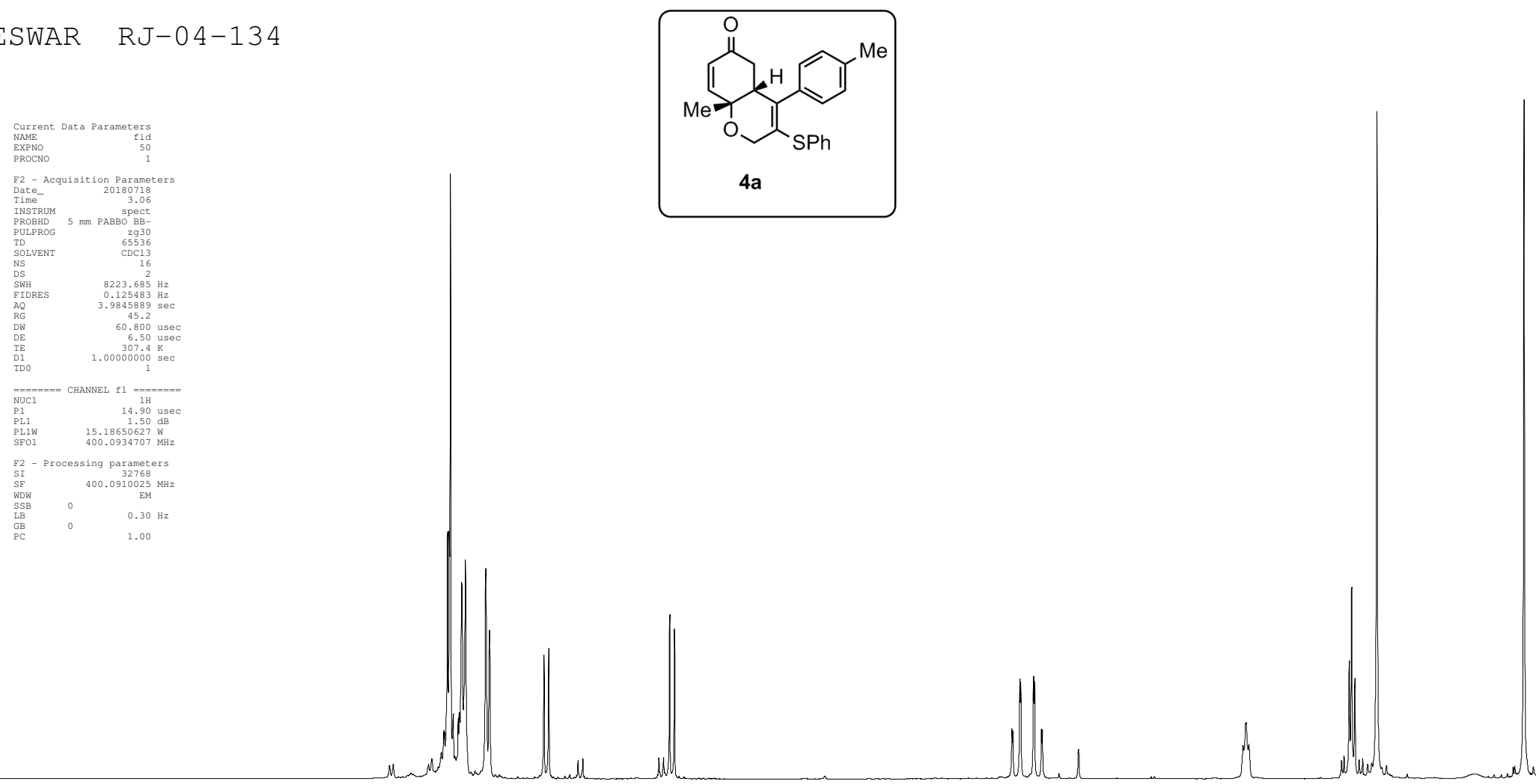

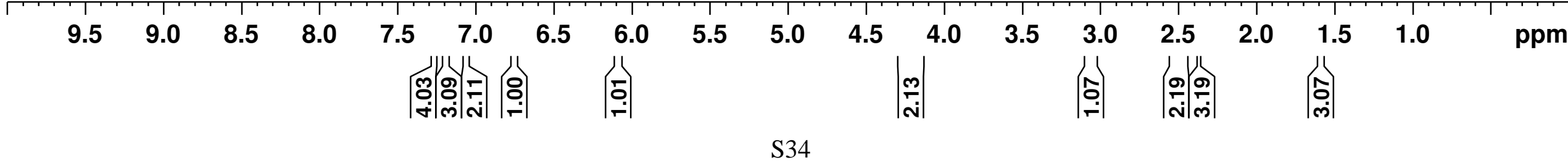



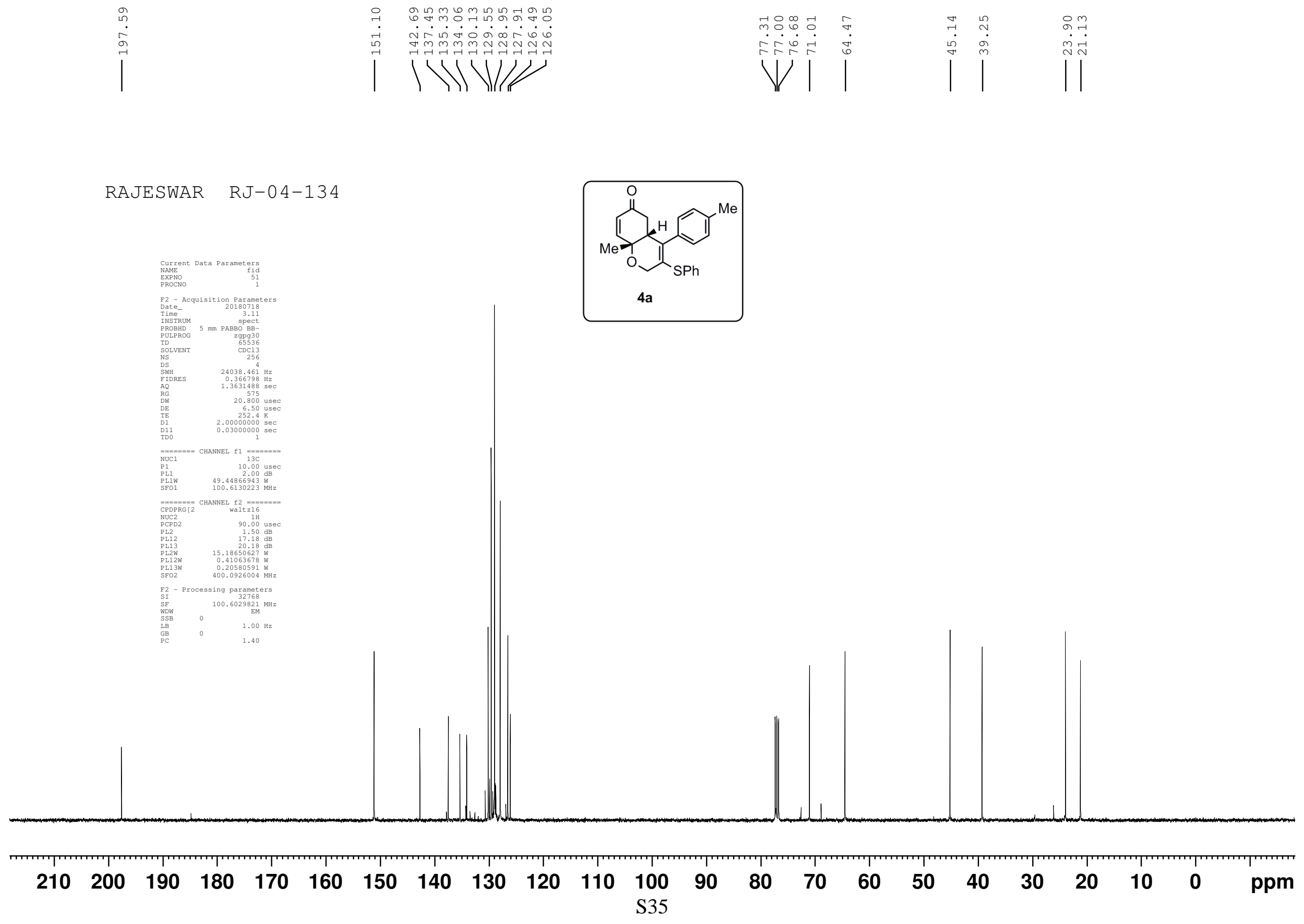


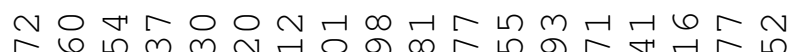

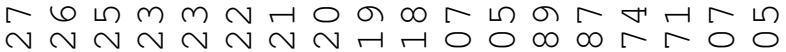
-

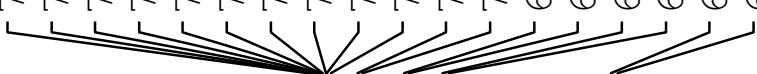

अ

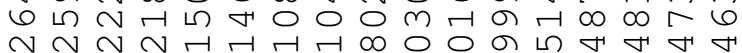
$\dot{\sigma} \dot{\sigma} \dot{\gamma} \dot{\gamma} \dot{\gamma} \dot{\sigma} \dot{\sigma} \dot{m} \dot{m} \dot{m} \dot{\sim} \dot{\sim} \dot{\sim} \dot{\sim}$ 3

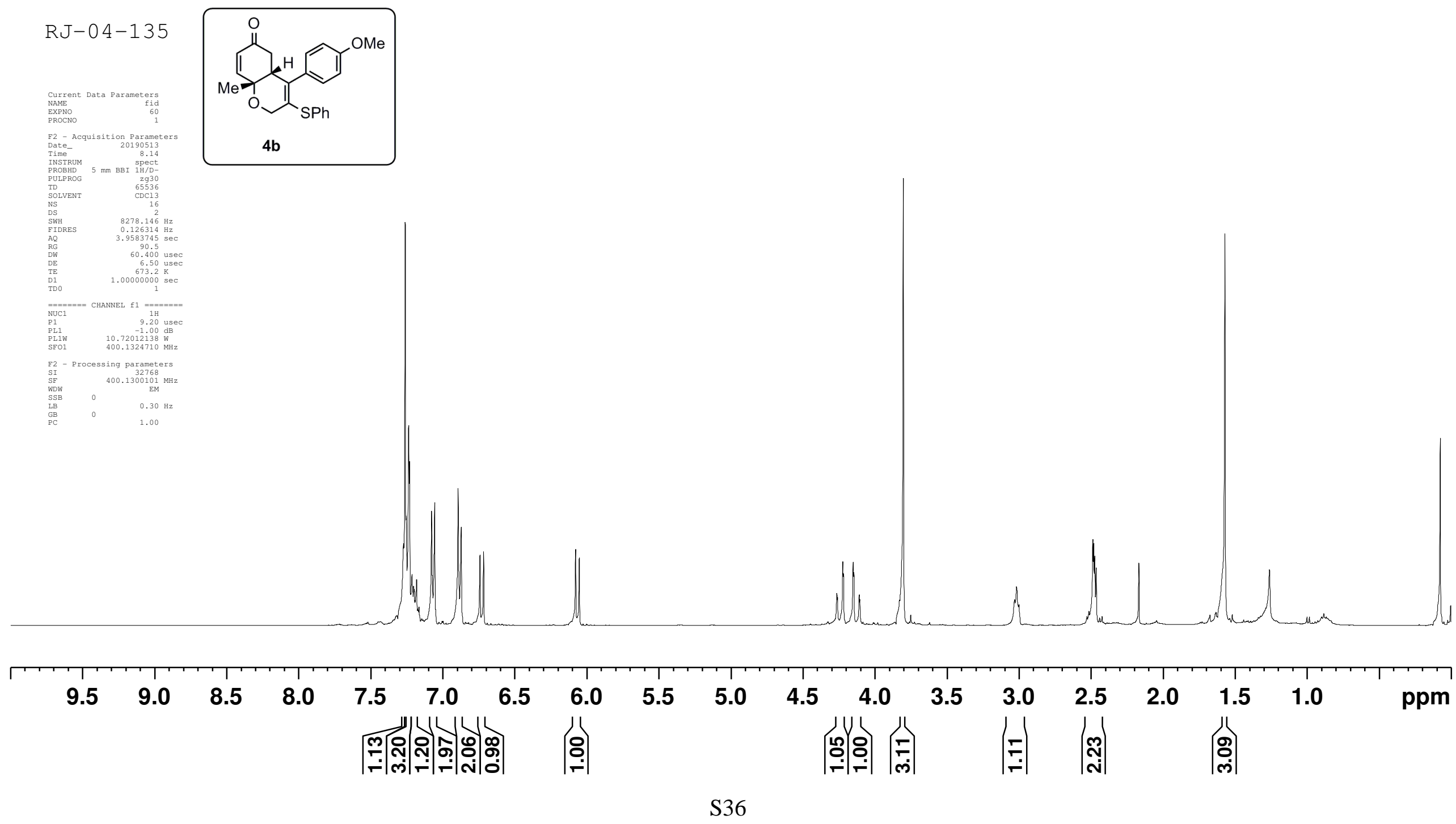




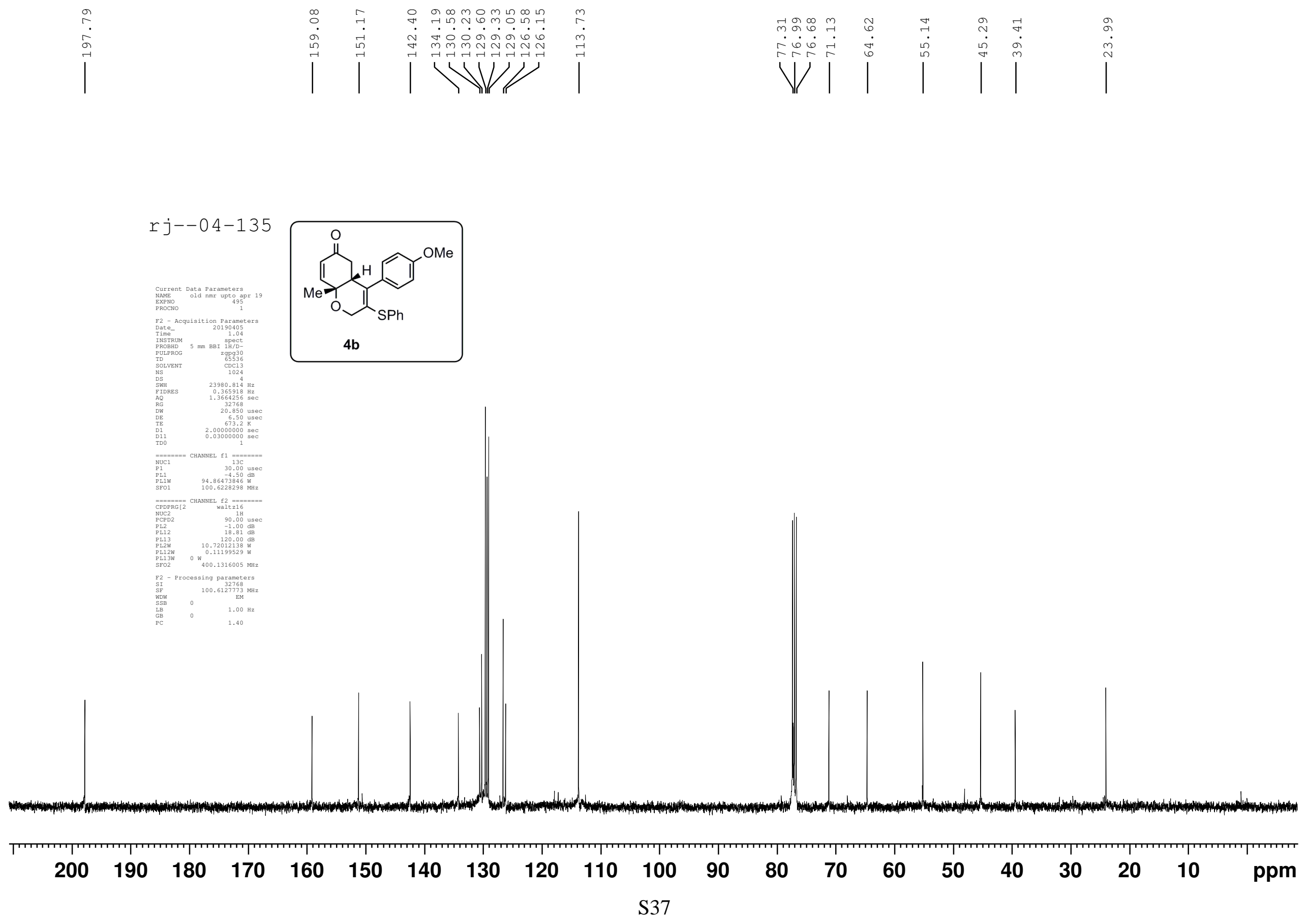



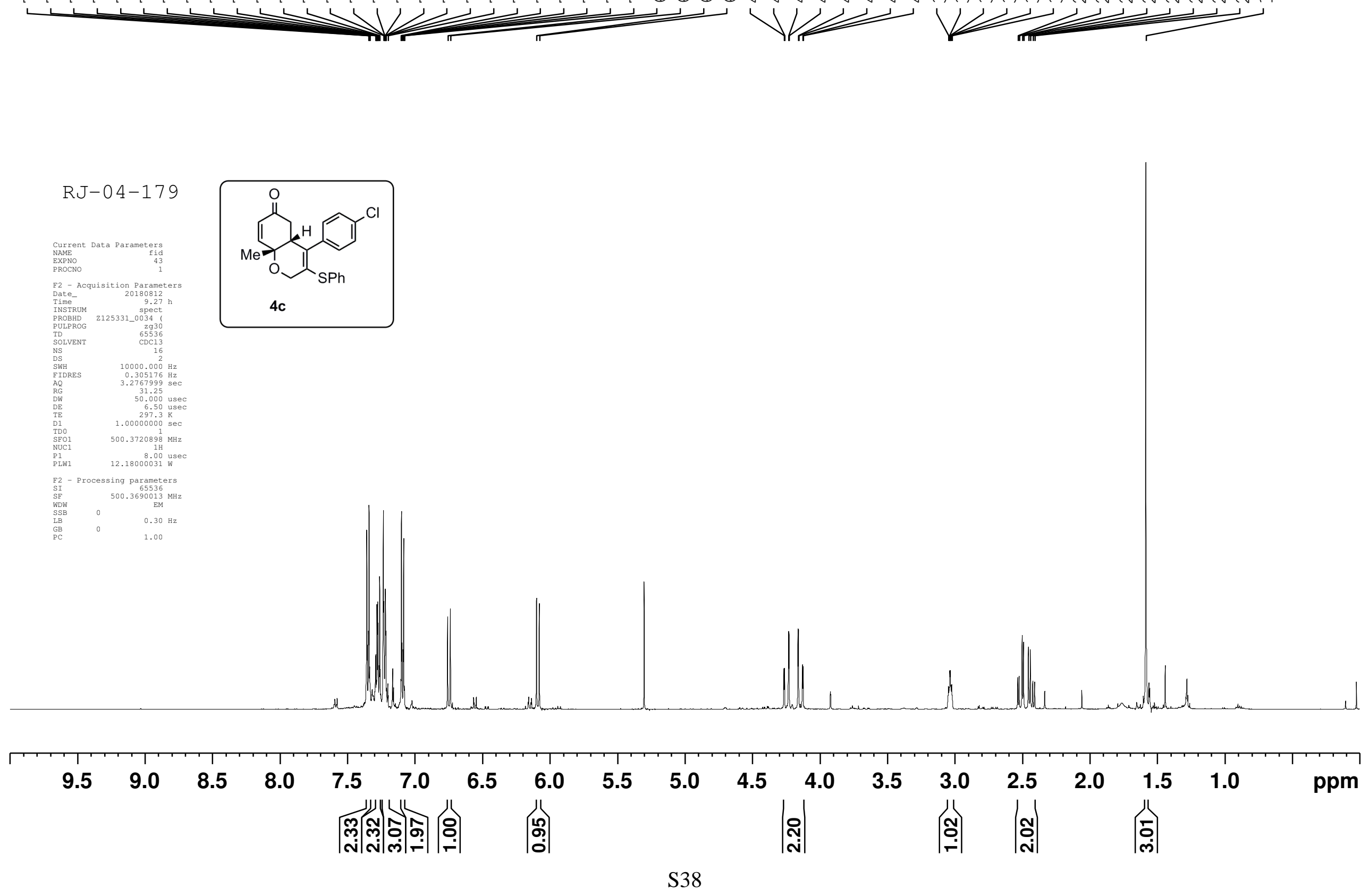


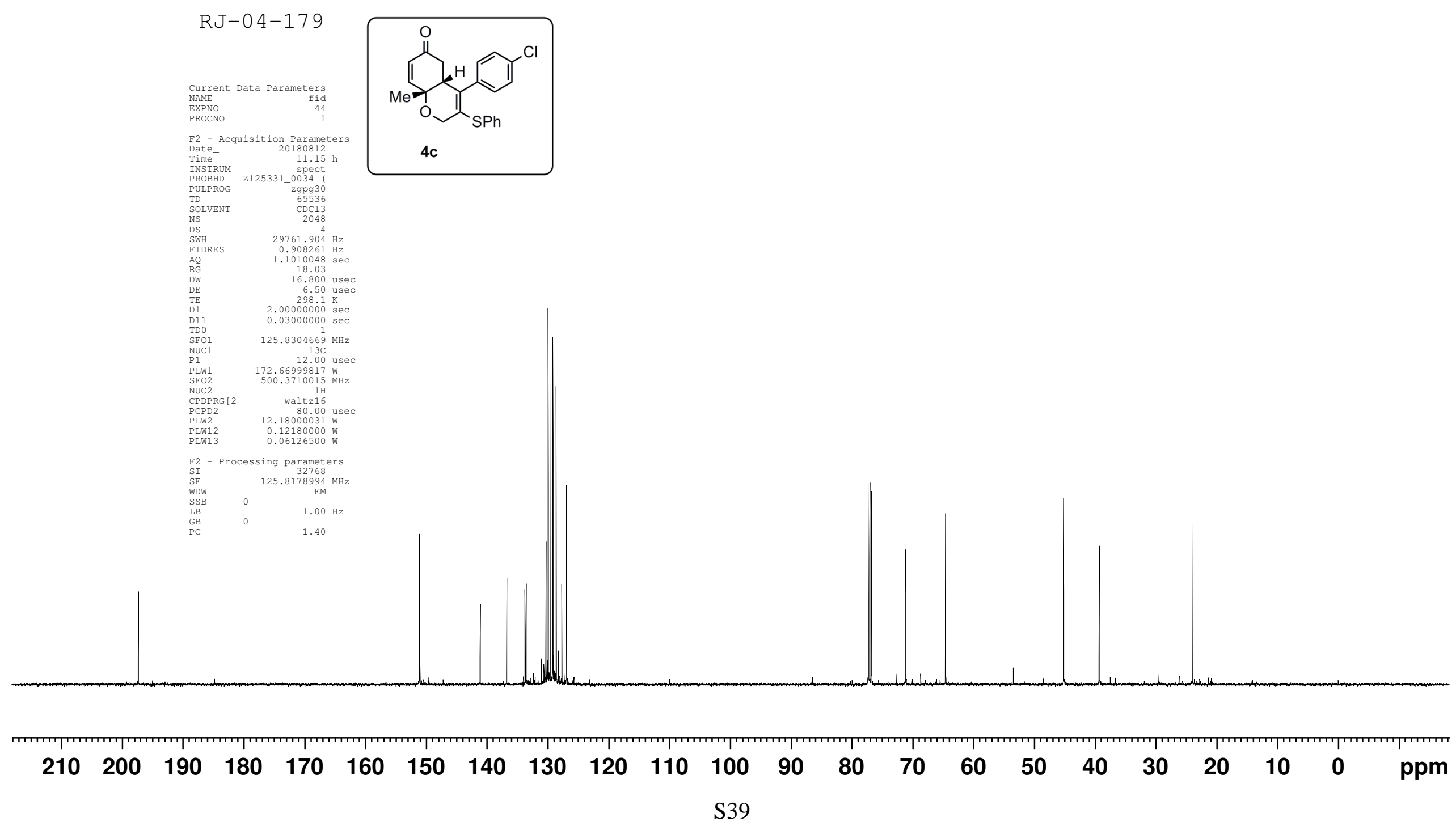


RAJESWAR RJ-04-147
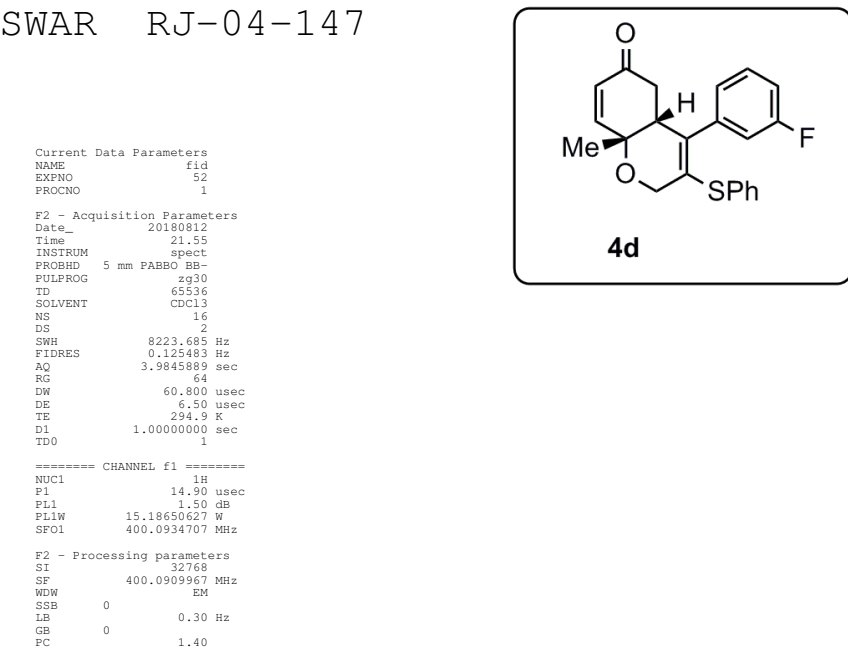

$4 d$
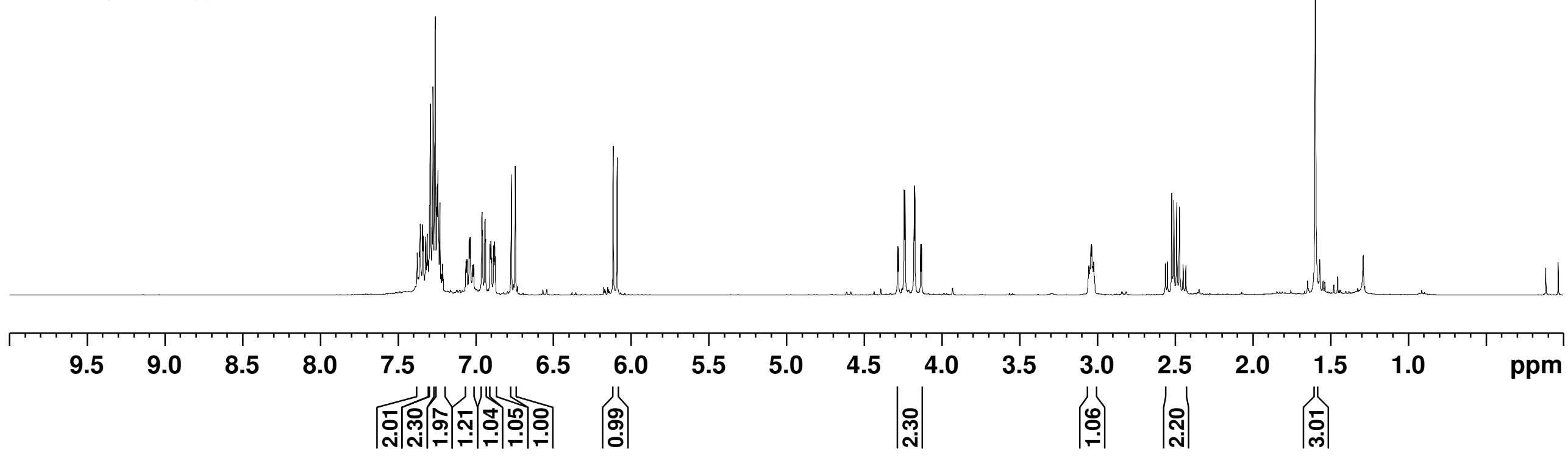


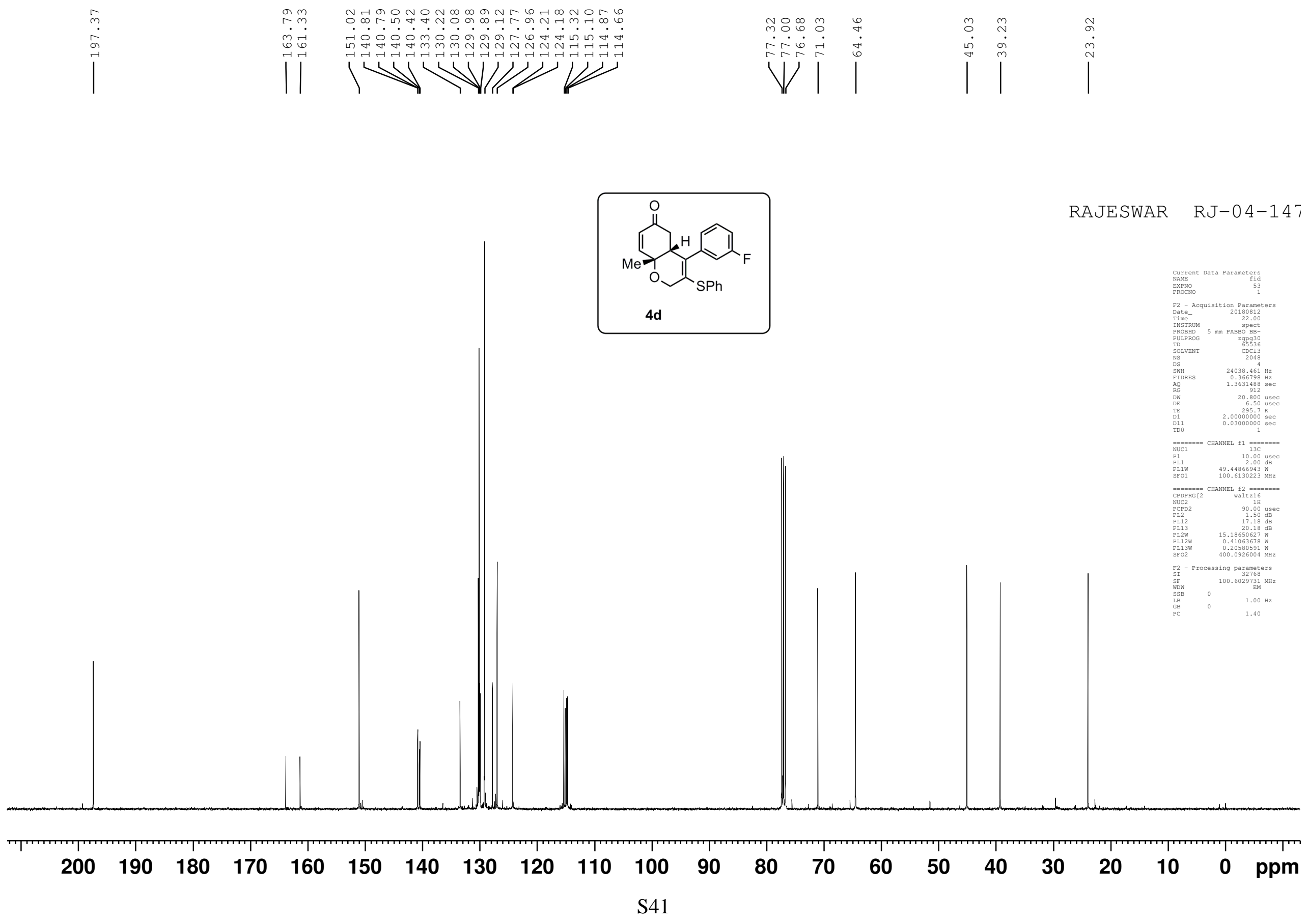




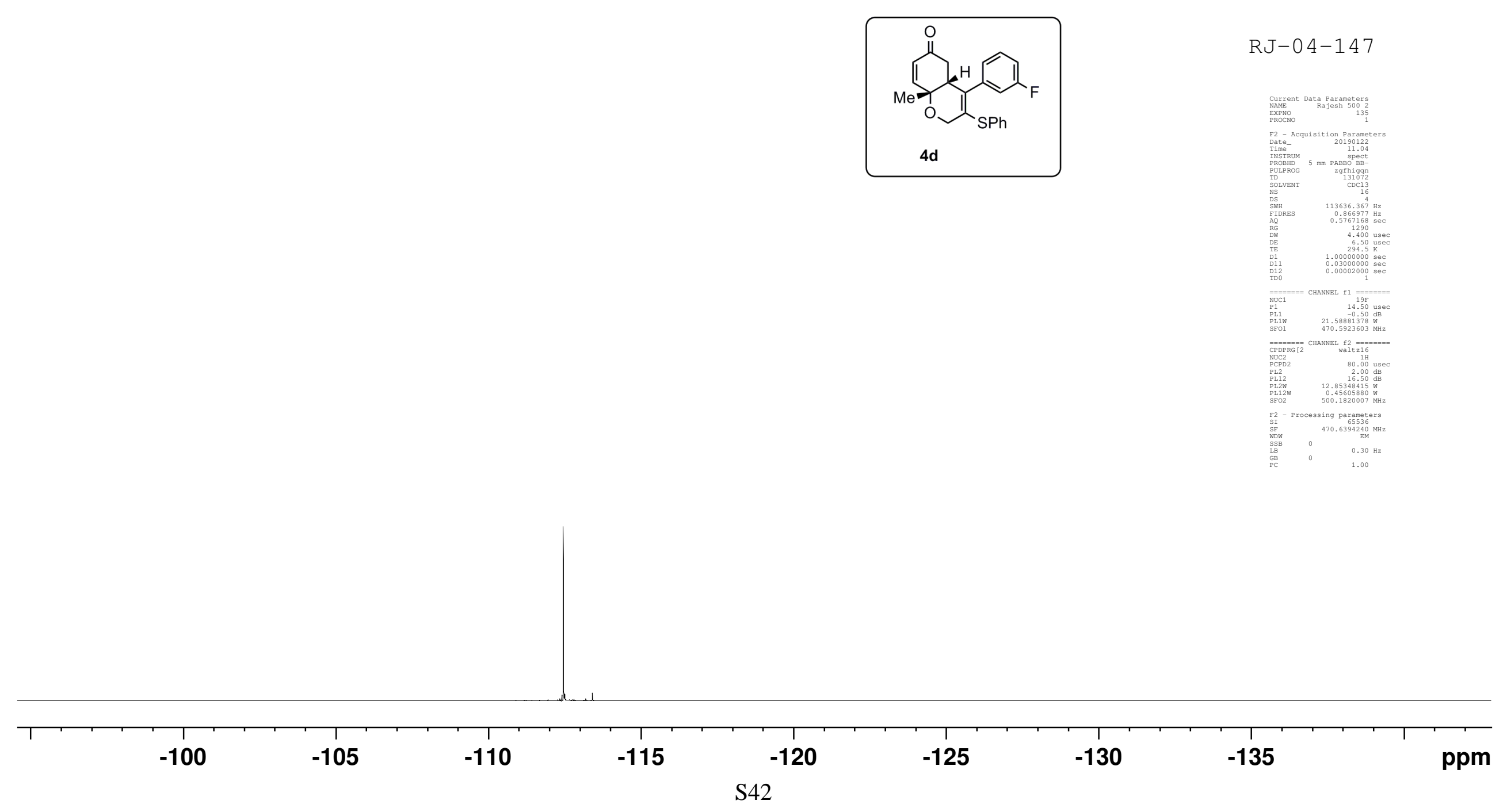




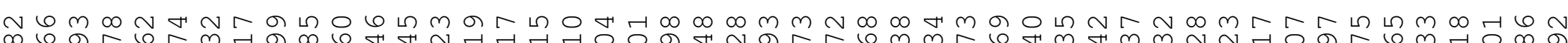
虽

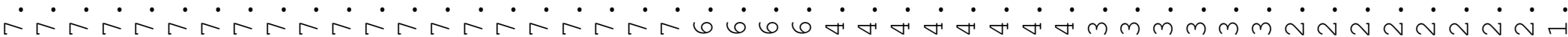

L

RJ-04-178
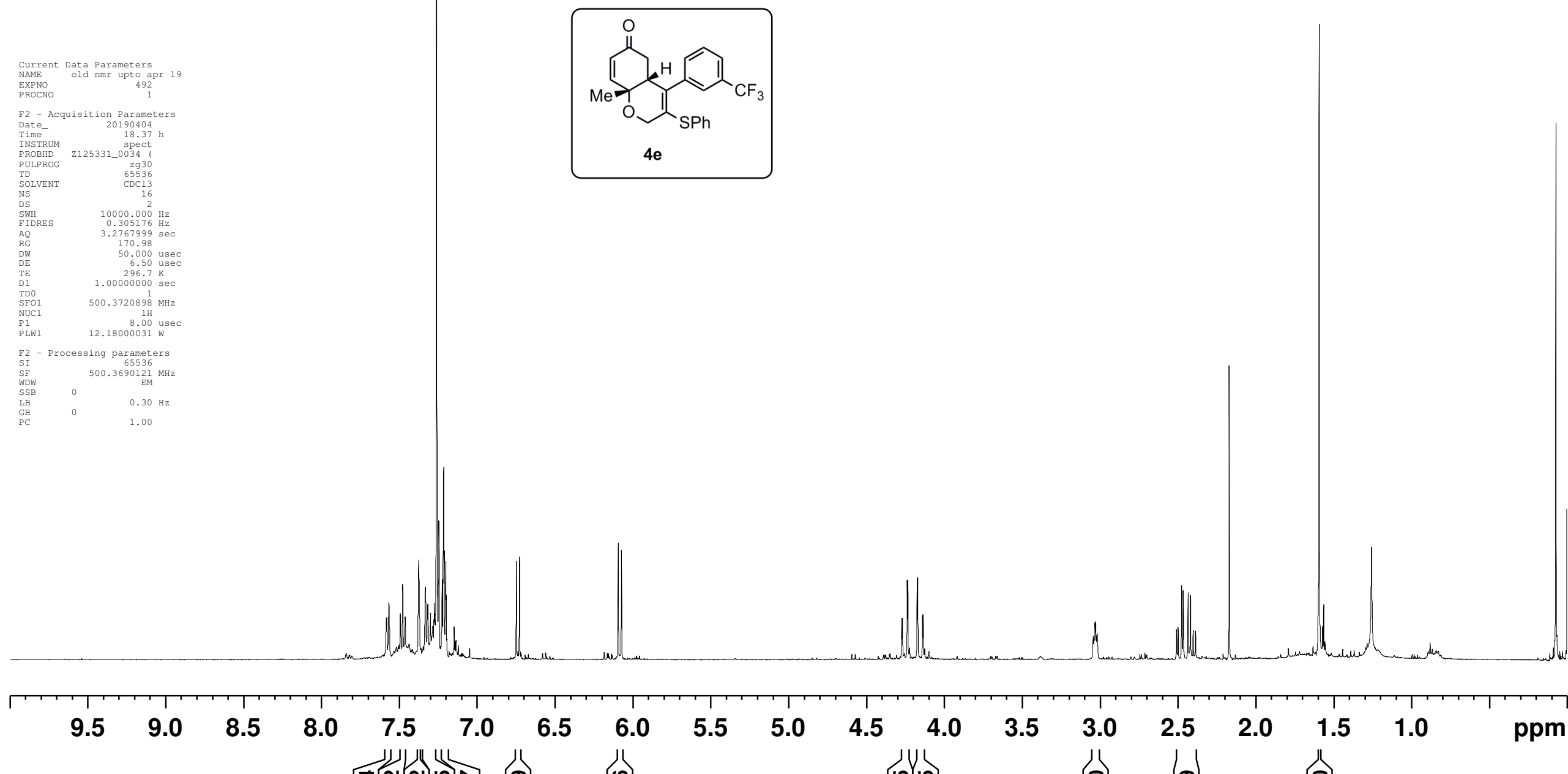

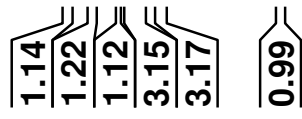
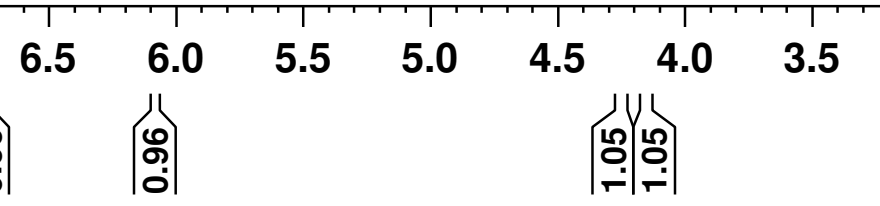

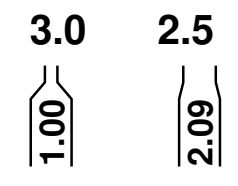



$1.5 \quad 1.0$

ppm 


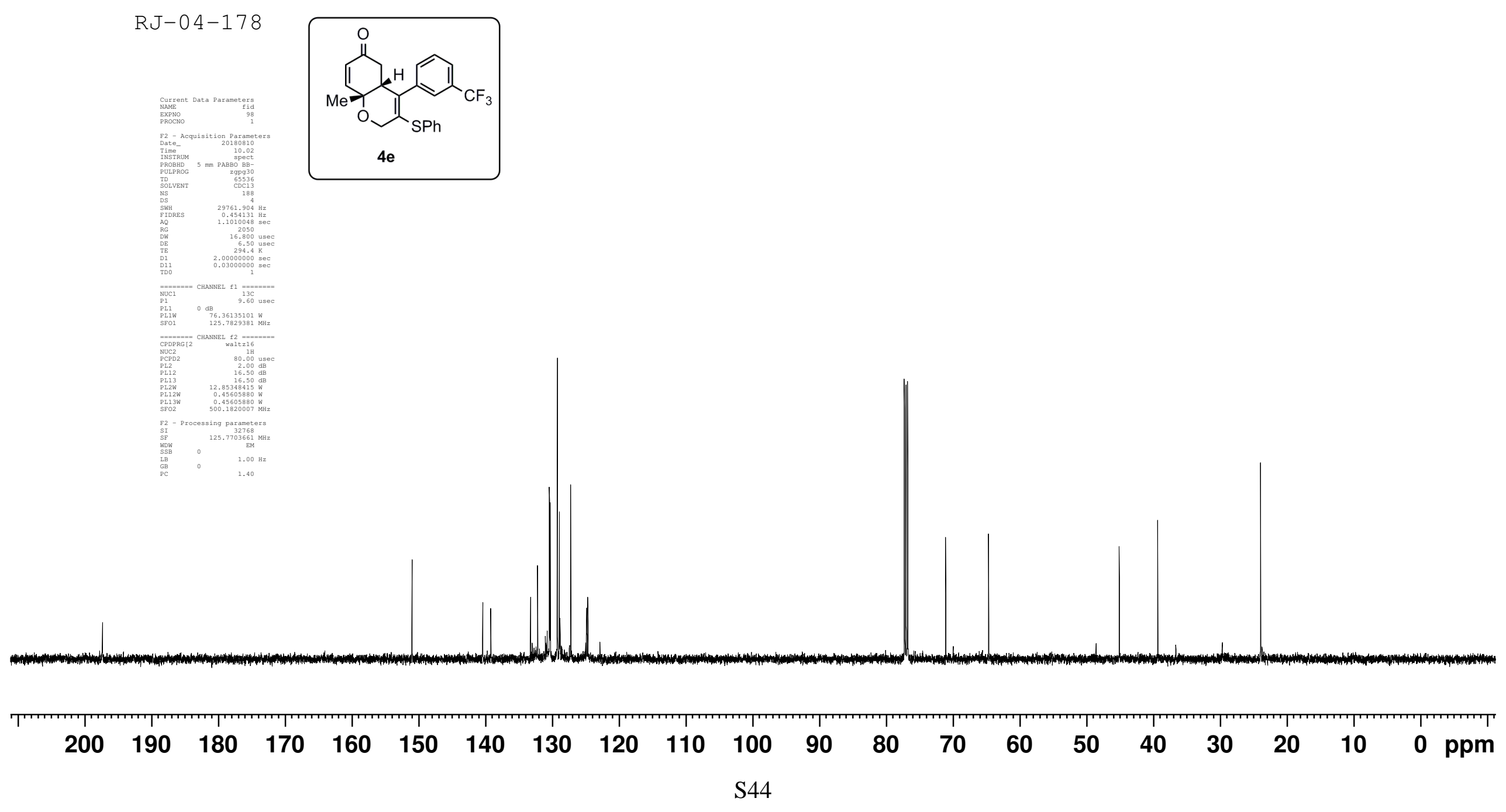




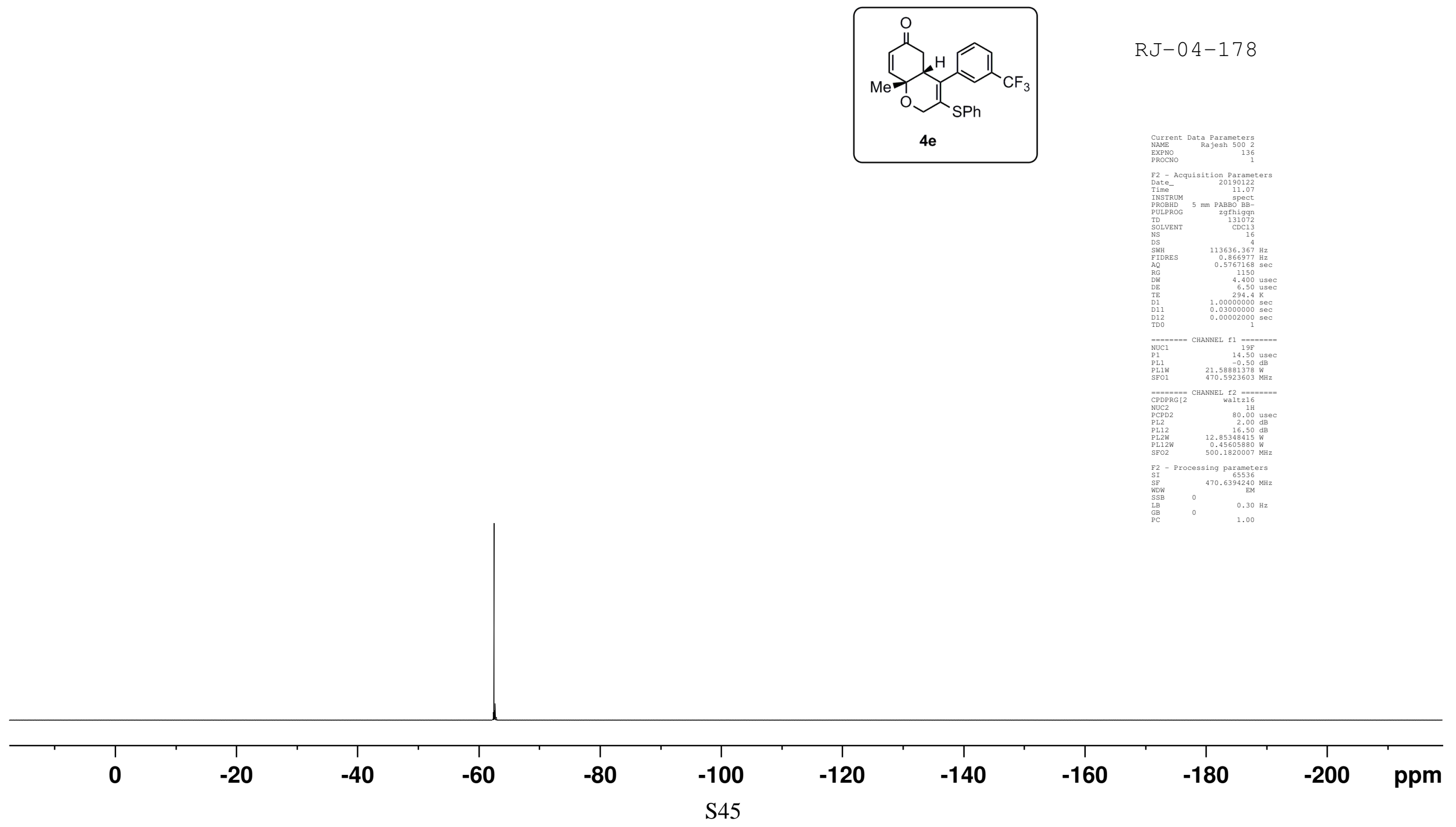




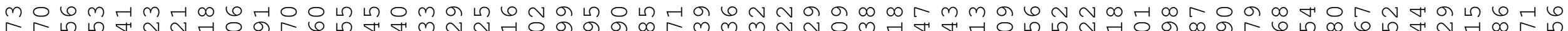

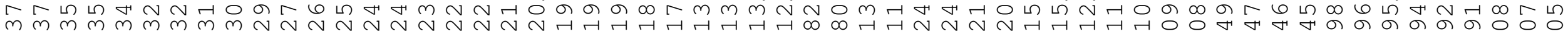

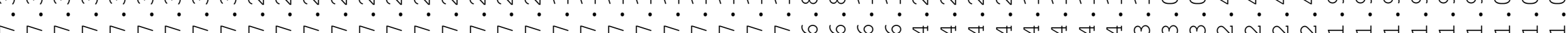



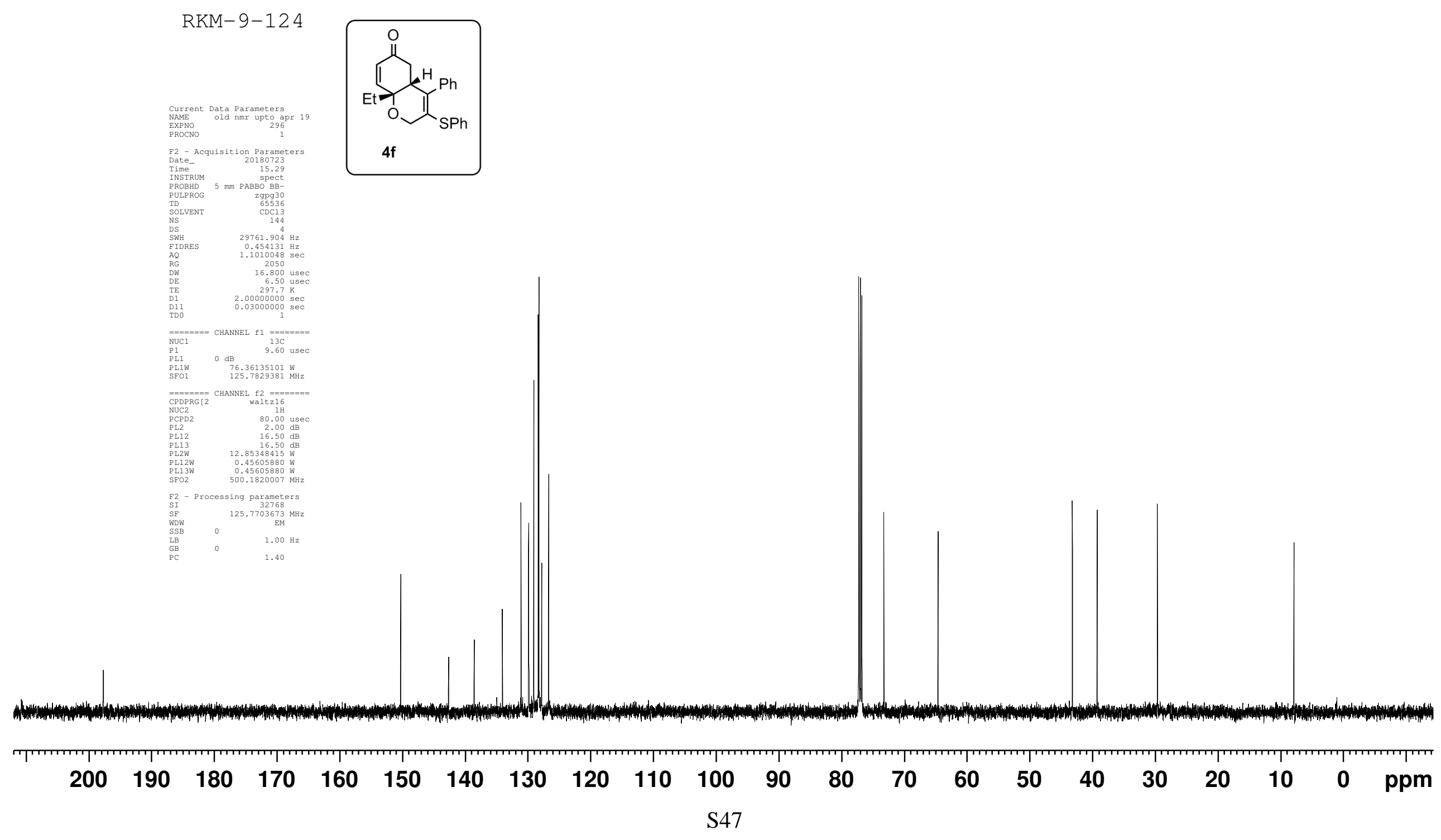




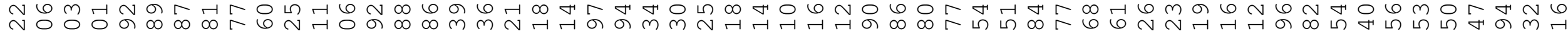

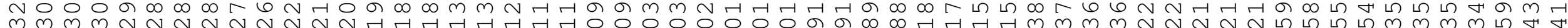

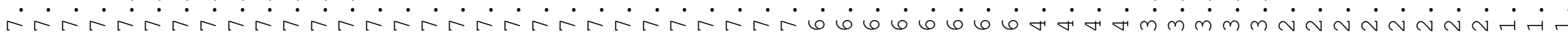

(n)
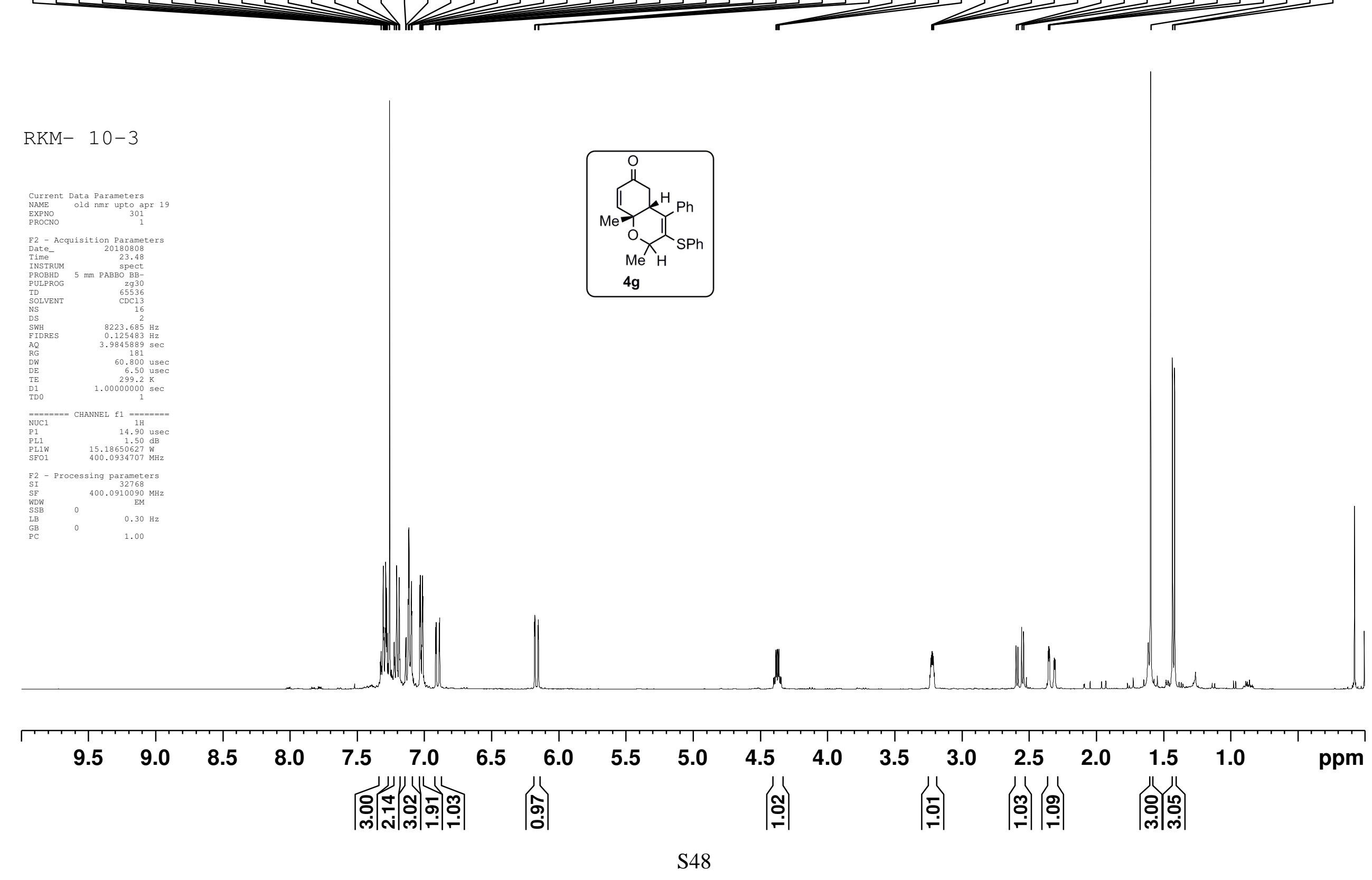


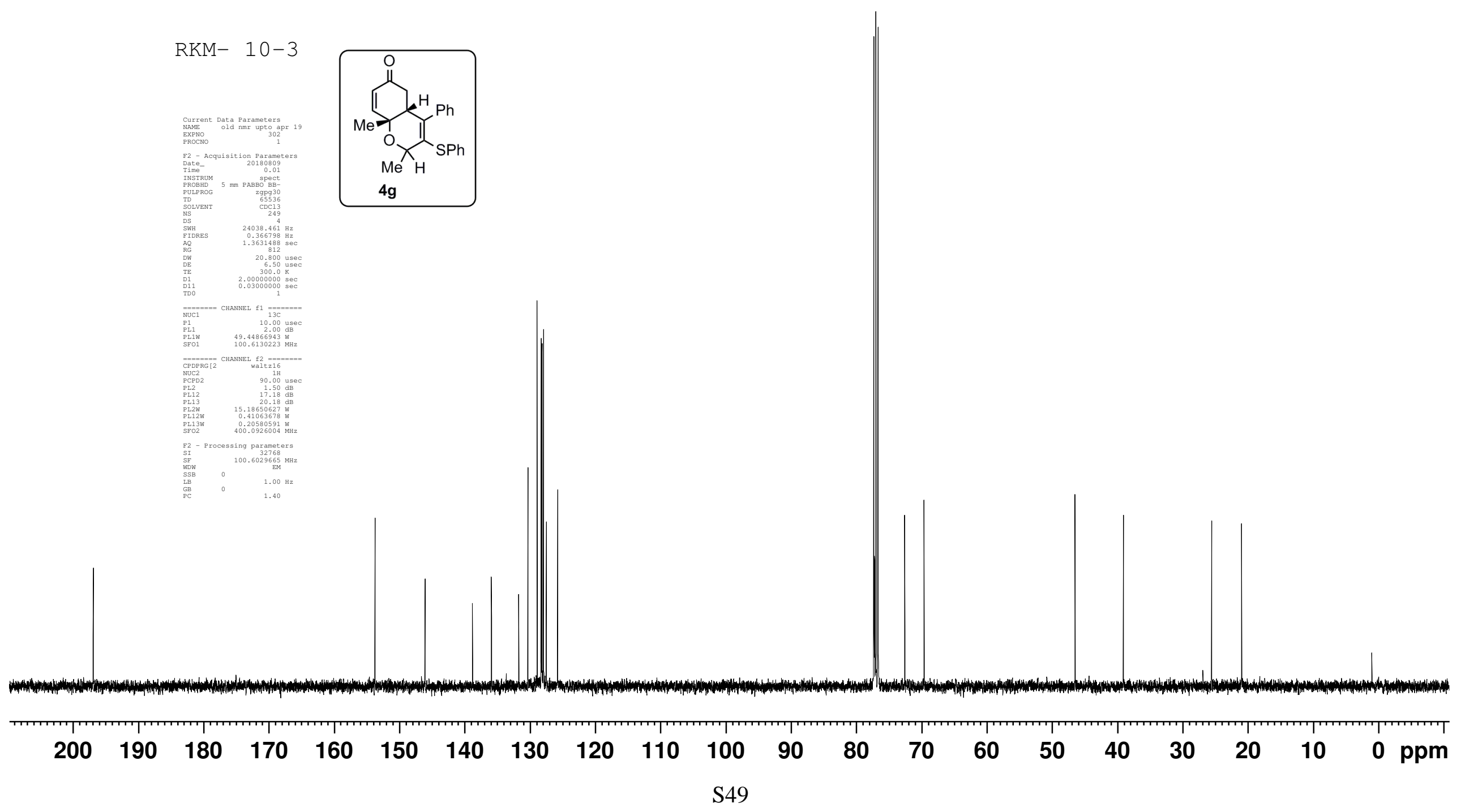


のの $\backsim m \sim r m \infty m r$

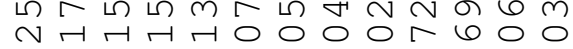

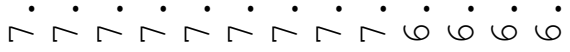

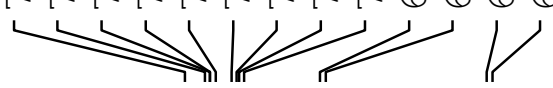

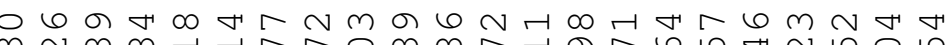

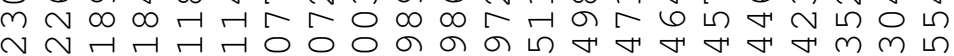
$\dot{\sigma} \dot{\sigma} \dot{\sigma} \dot{\sigma} \dot{\sigma} \dot{\sigma} \dot{\sigma} \dot{m} \dot{\sim} \dot{\sim} \dot{\sim} \dot{\sim} \dot{\sim} \dot{\sim} \dot{\sim} \dot{\sim} \dot{\sim} \dot{\sim} \dot{\sim}$

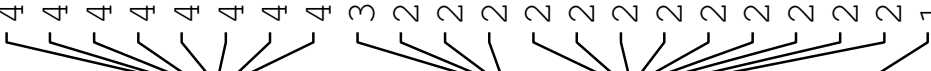
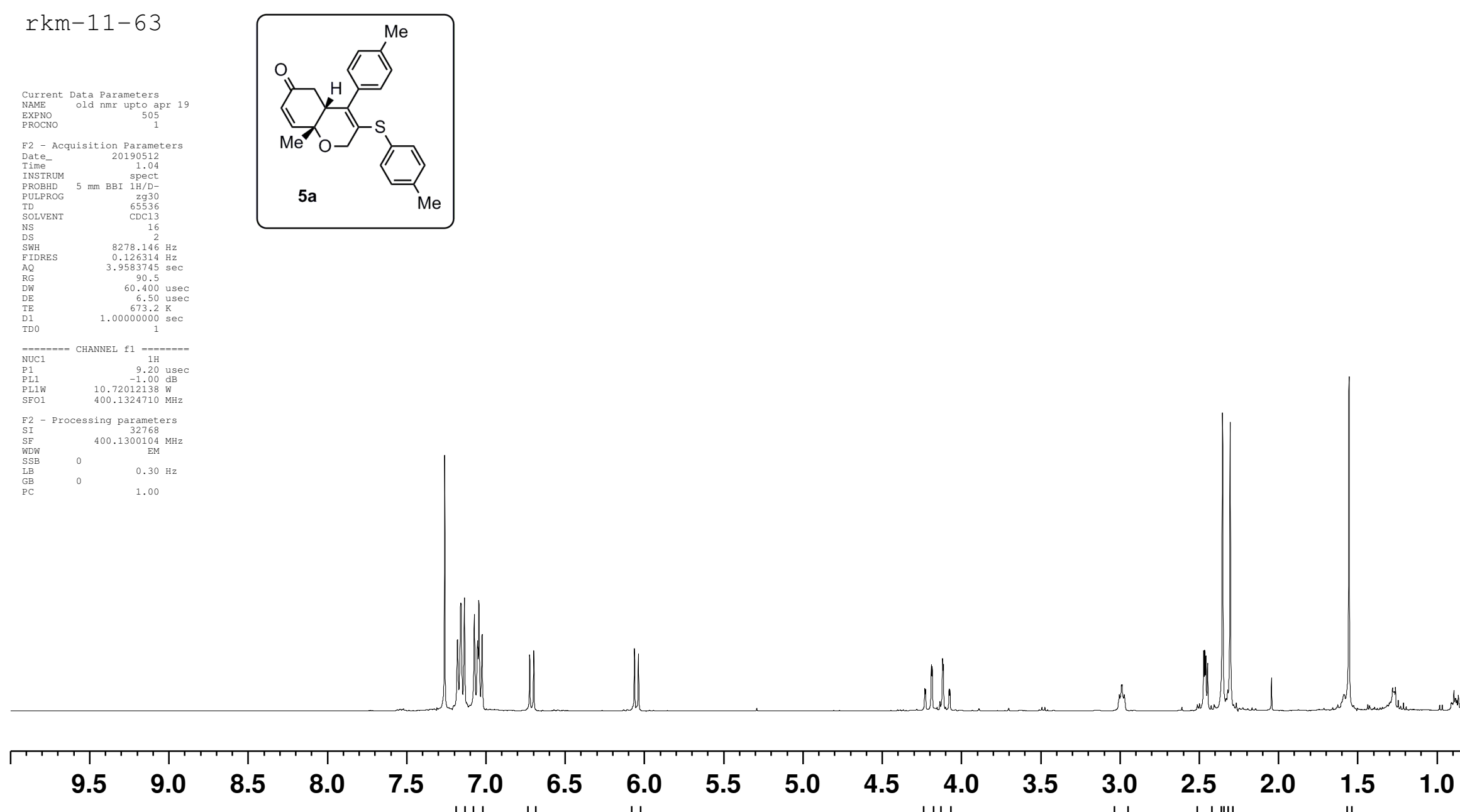



$5.5 \quad 5.0$

$\begin{array}{lll}4.5 & 4.0 & 3.5\end{array}$

3.02

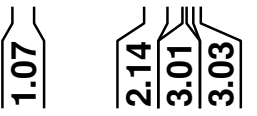

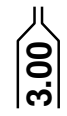


RKM-11-63
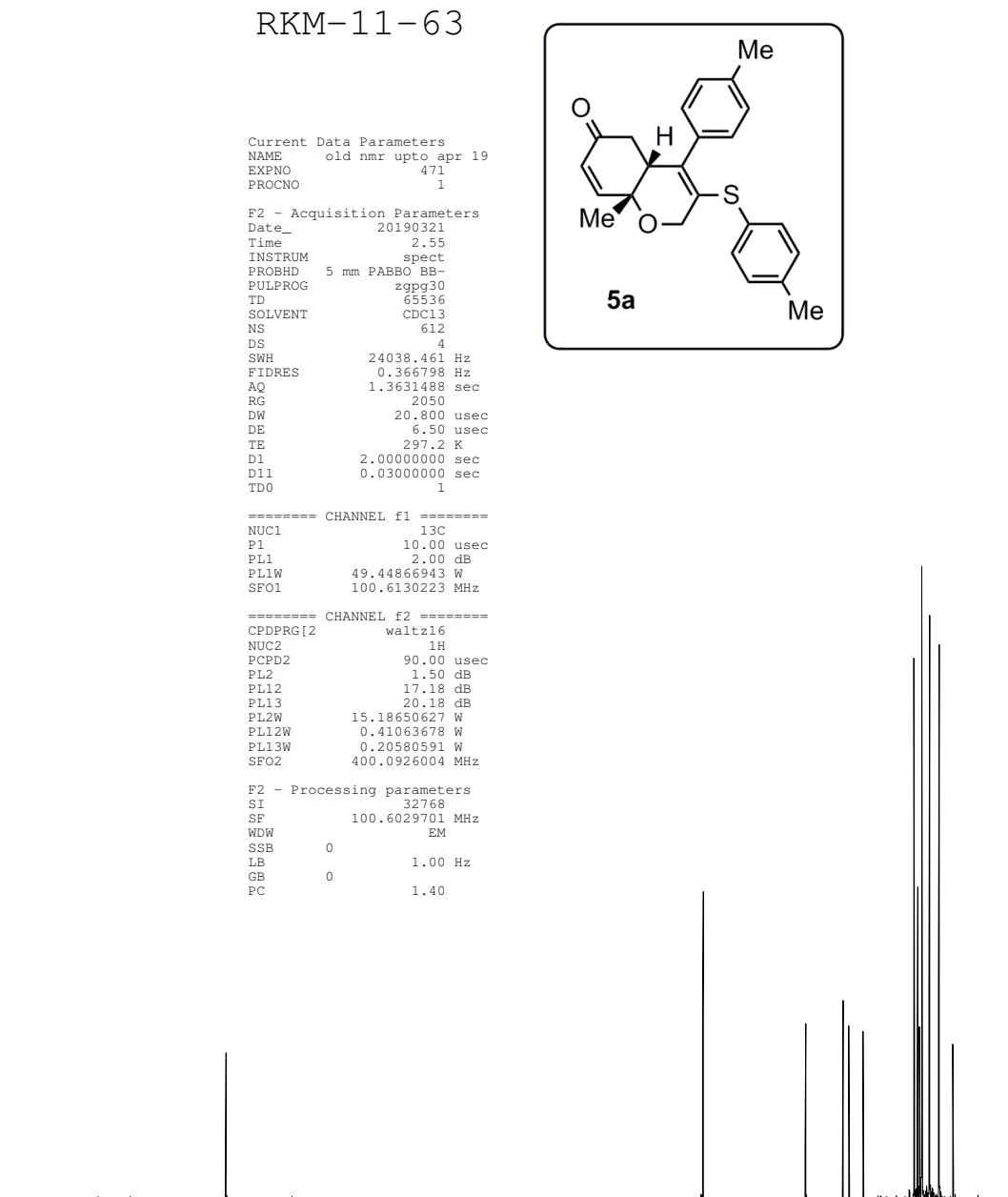

$$
210200
$$$$
1901
$$

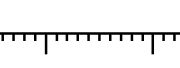

160150

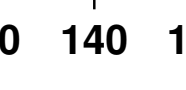


$\circ m m m m \circ \infty\llcorner\sigma r N$

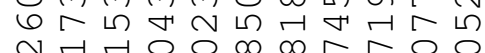
-

III
ש

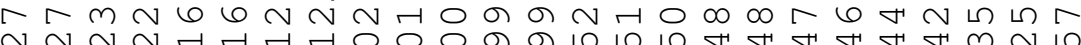

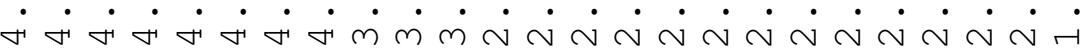

$r k m-11-64$
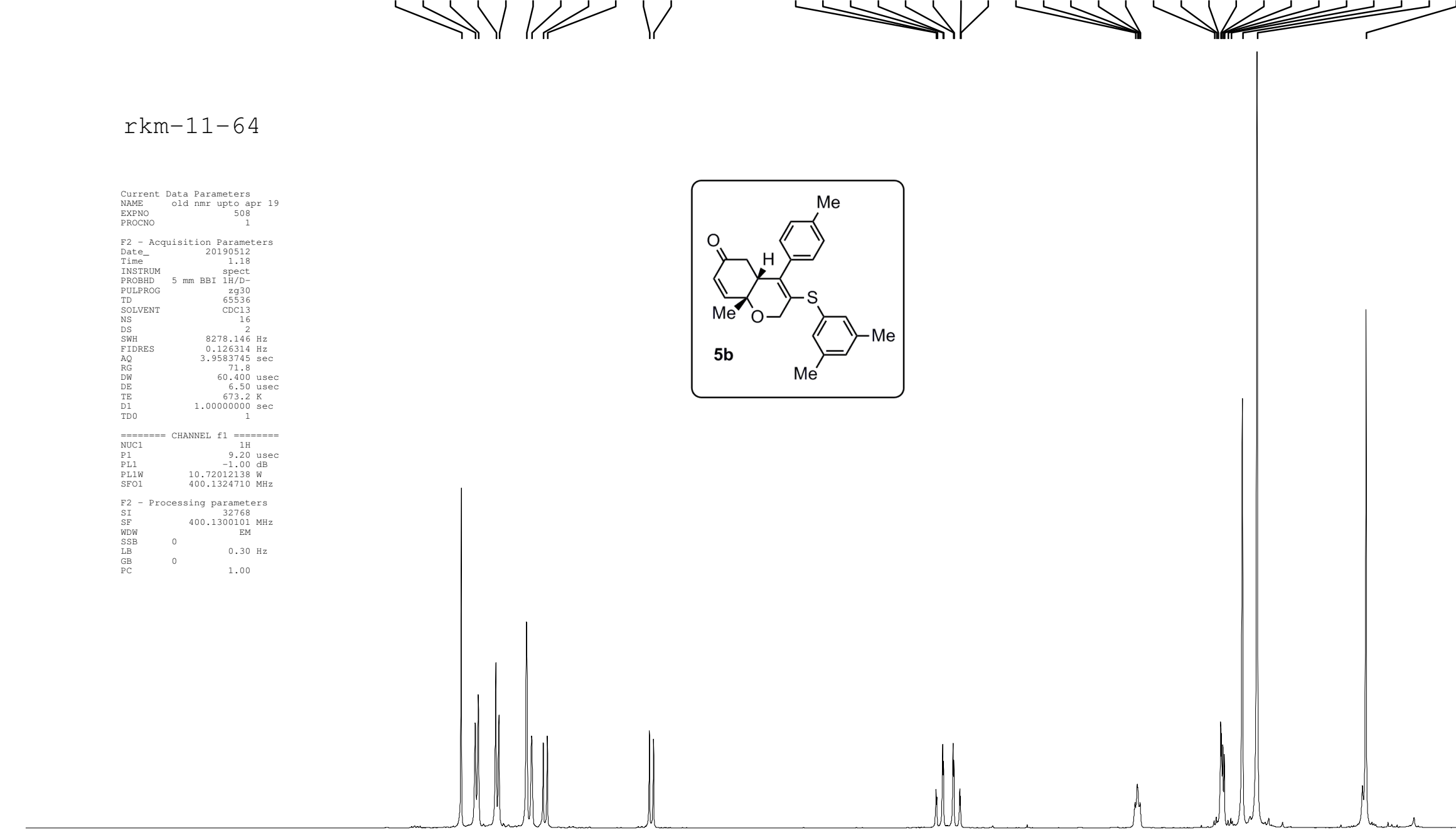

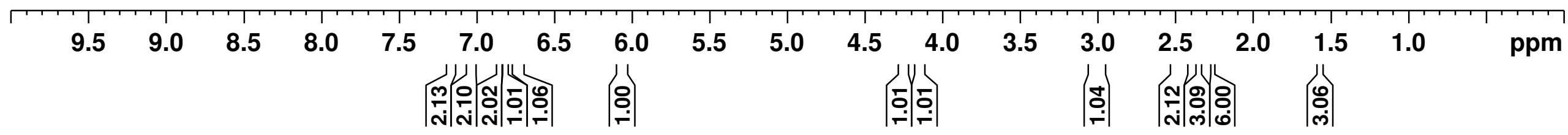


RKM-11-64

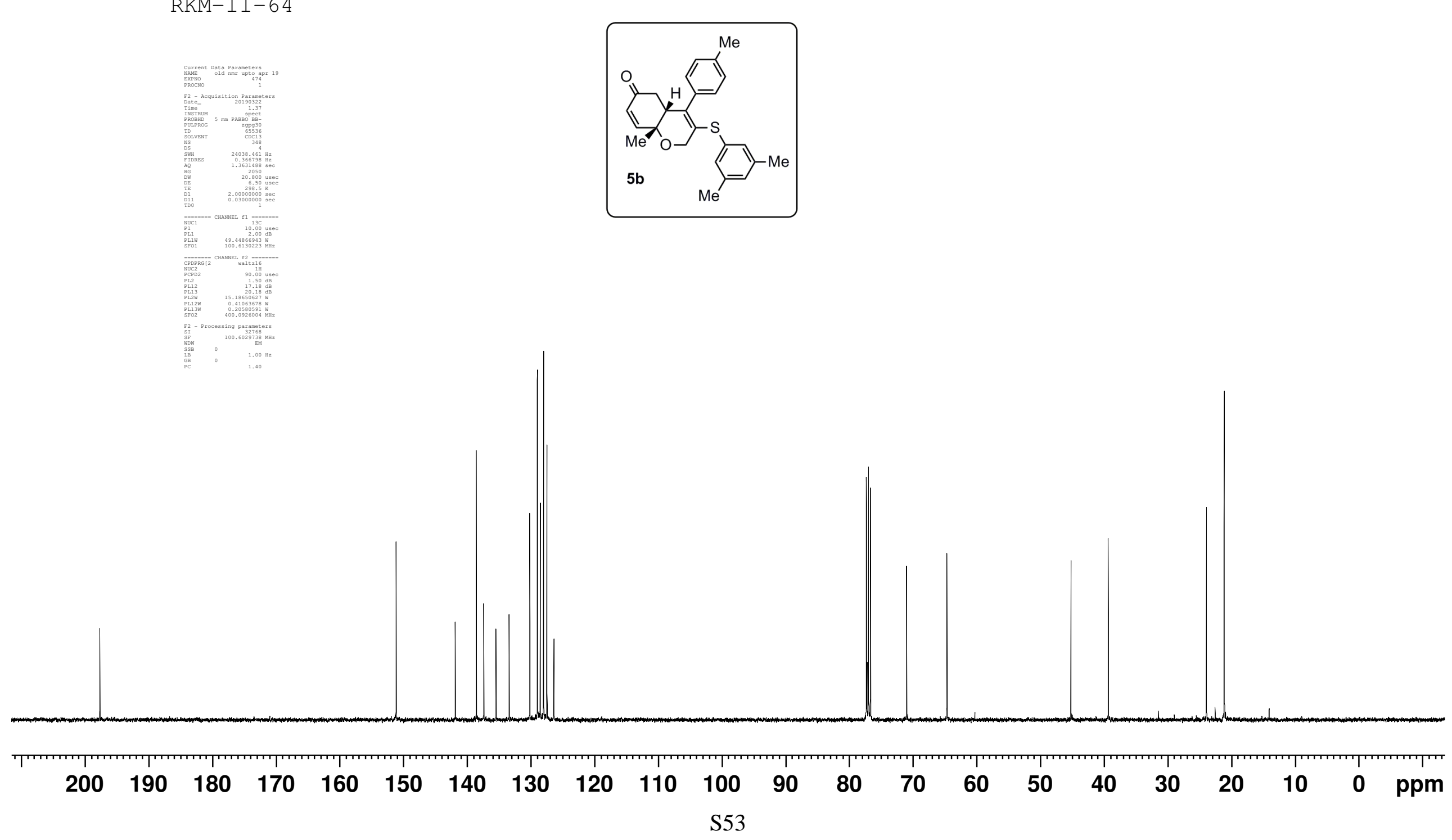


$r k m-11-65$
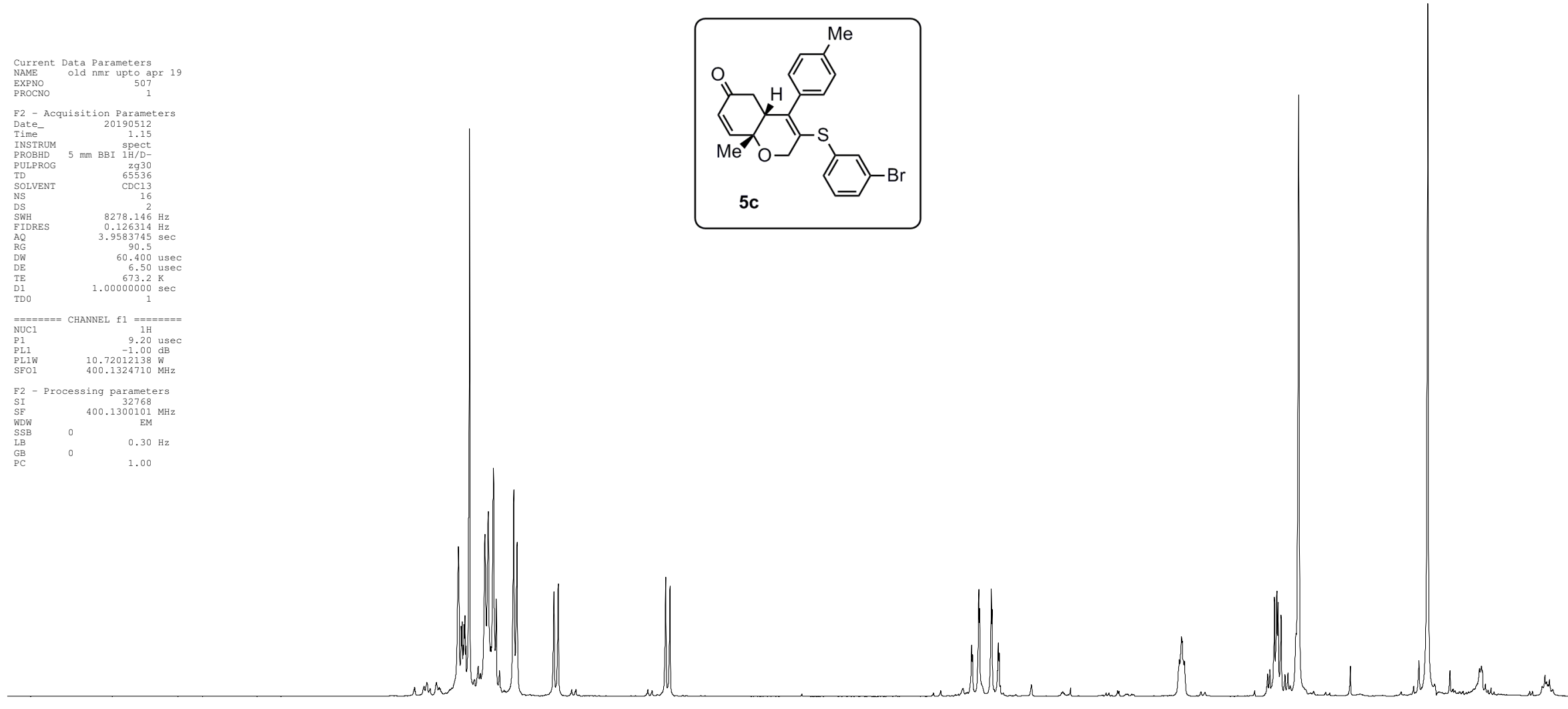

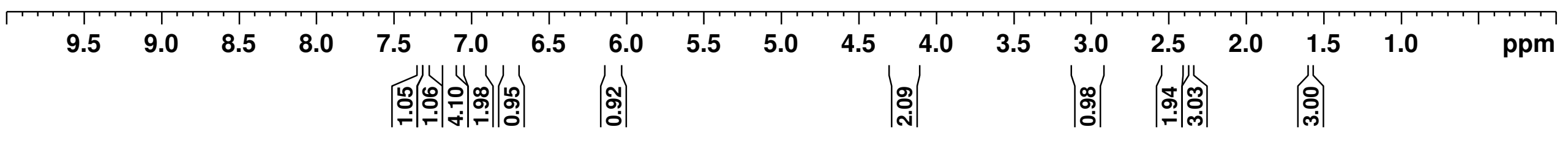


$r k m-11-65$
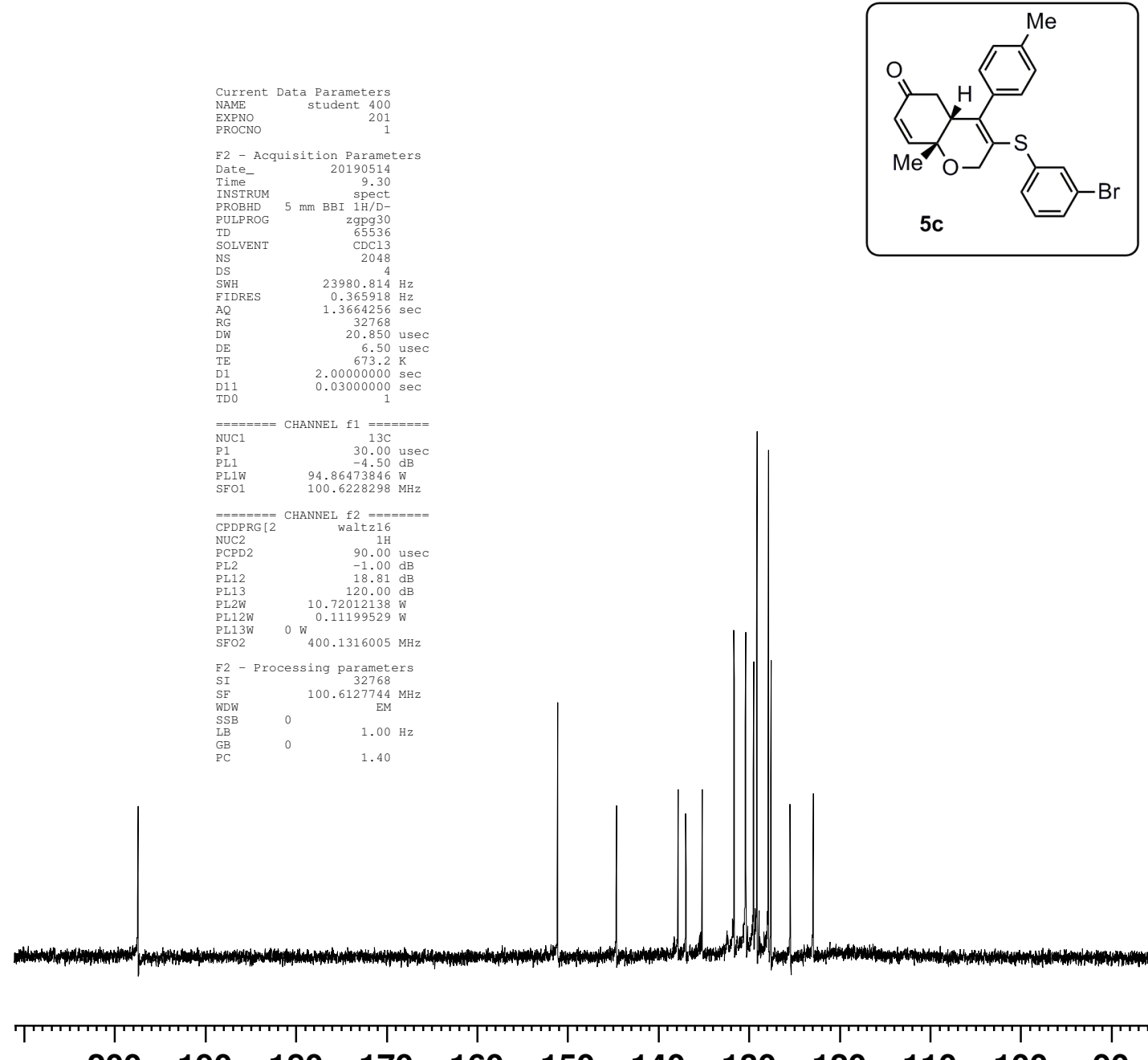


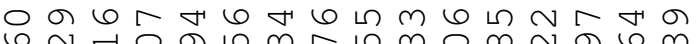
$\sim \sim \sim \sim$ ก

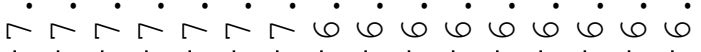
$\longrightarrow$ m

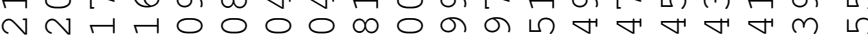
$\dot{\nabla} \dot{\sigma} \dot{\nabla} \dot{\sigma} \dot{\sigma} \dot{\sigma} \dot{\sigma} \dot{m} \dot{\sim} \dot{\sim} \dot{\sim} \dot{\sim} \dot{\sim} \dot{\sim} \dot{\sim} \dot{\sim}$

$\longrightarrow$

$r \mathrm{~km}-11-38$
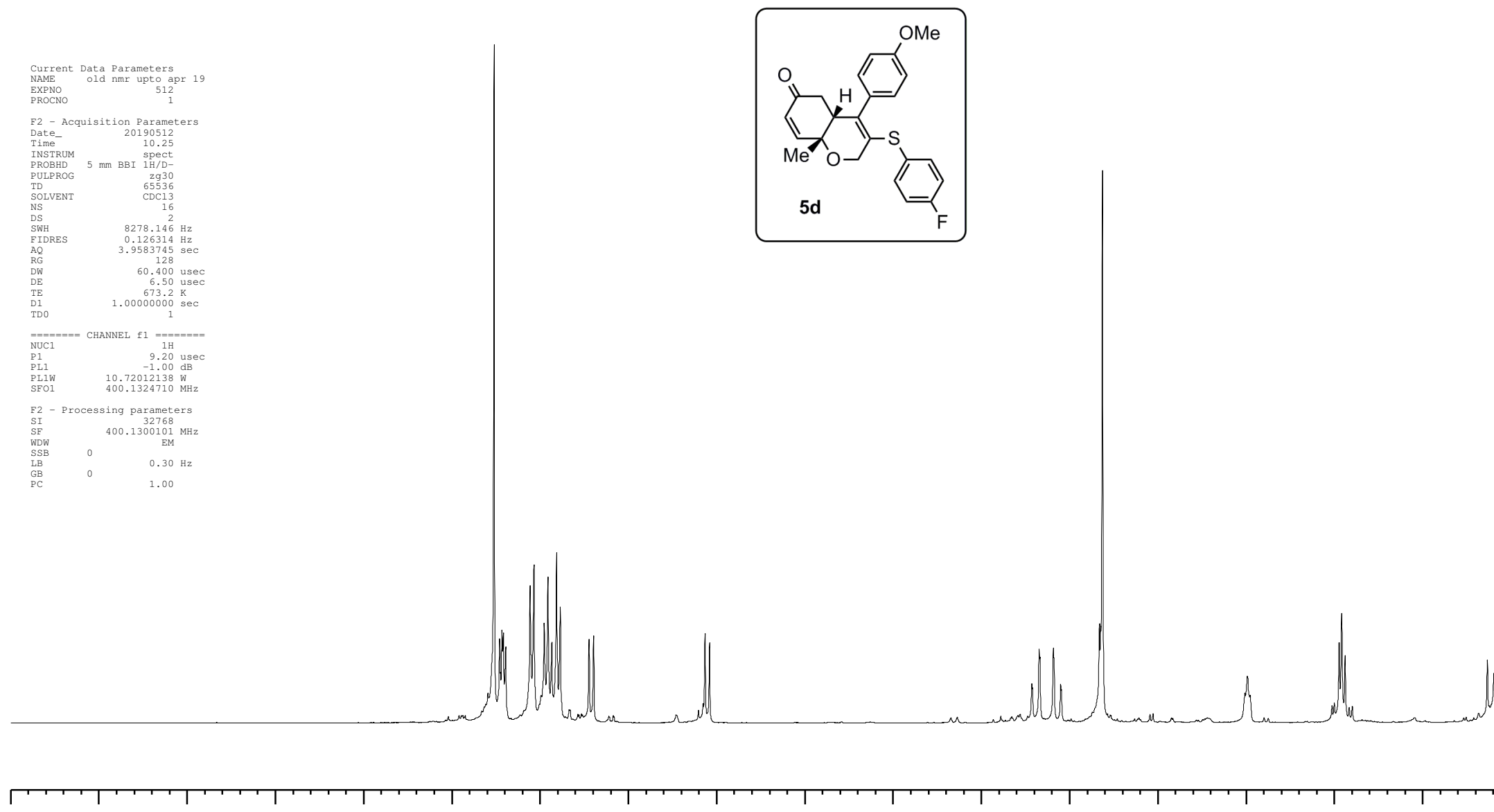

$9.5 \quad 9.0$

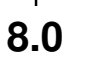

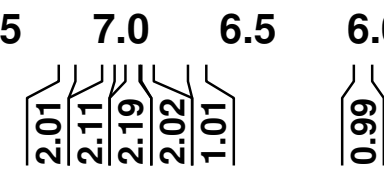

o.

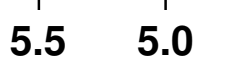

4.54 .0

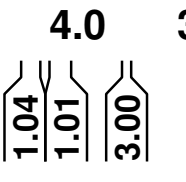

3.0

2.5

$2.0 \quad 1.5$

$\left|\begin{array}{l}0 \\ \hdashline \\ i\end{array}\right| \quad\left|\begin{array}{c}\mathbf{N} \\ \bar{N}\end{array}\right|$

$\left|\begin{array}{l}0 \\ 0 \\ \dot{m}\end{array}\right|$ 

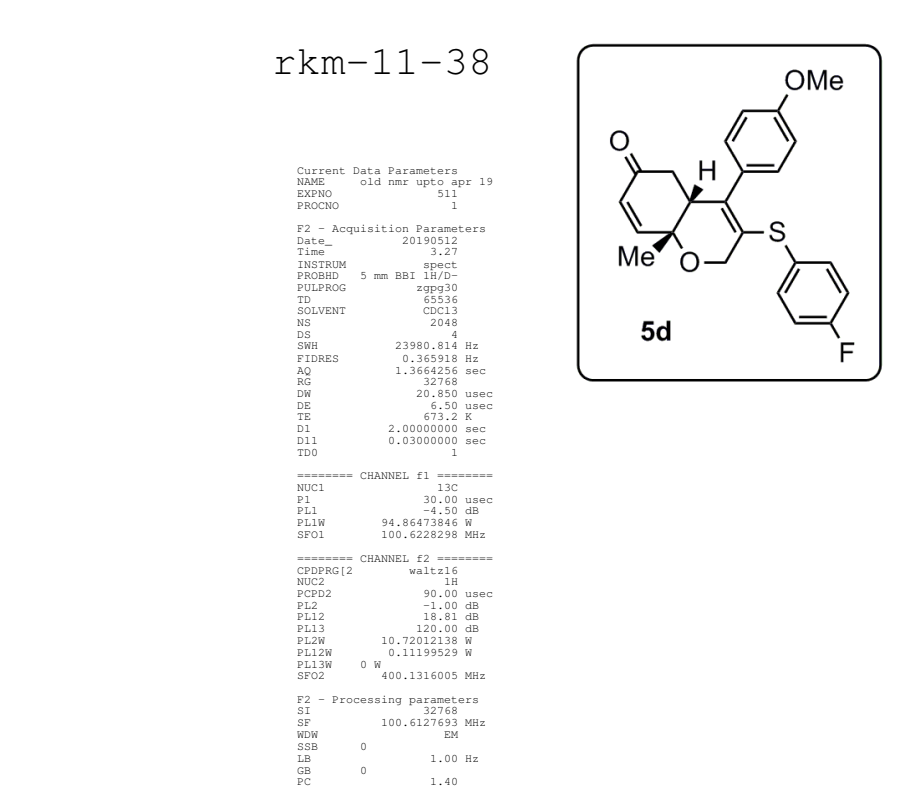


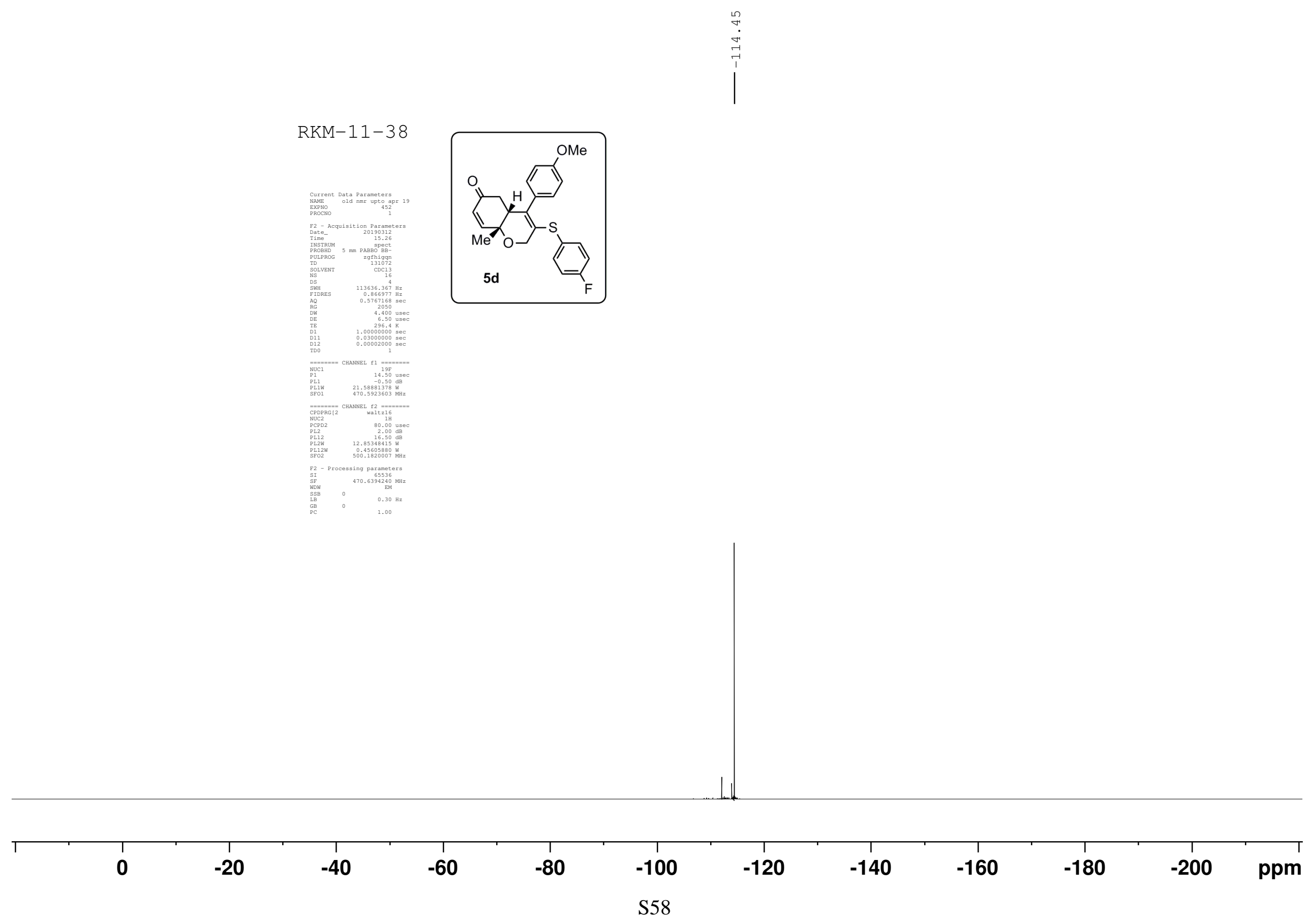




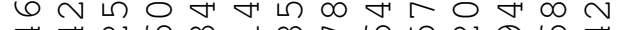

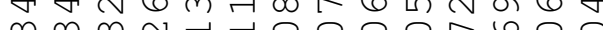

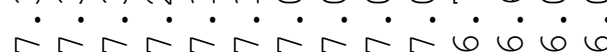

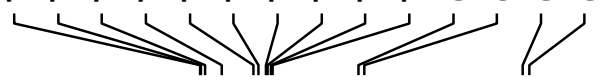

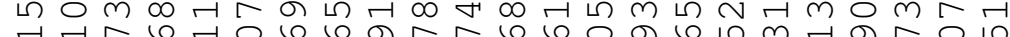

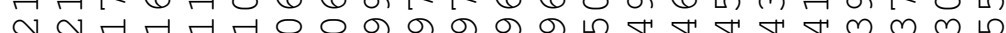
$\dot{\gamma} \dot{\gamma} \dot{\gamma} \dot{\gamma} \dot{\gamma} \dot{\gamma} \dot{\gamma} \dot{\gamma} \dot{\sim} \dot{\sim} \sim \dot{\sim} \dot{\sim} \dot{\sim} \dot{\sim} \dot{\sim} \dot{\sim} \dot{\sim} \dot{\sim} \dot{\sim}$

$r \mathrm{~km}-11-39$


$\mathrm{Me}$
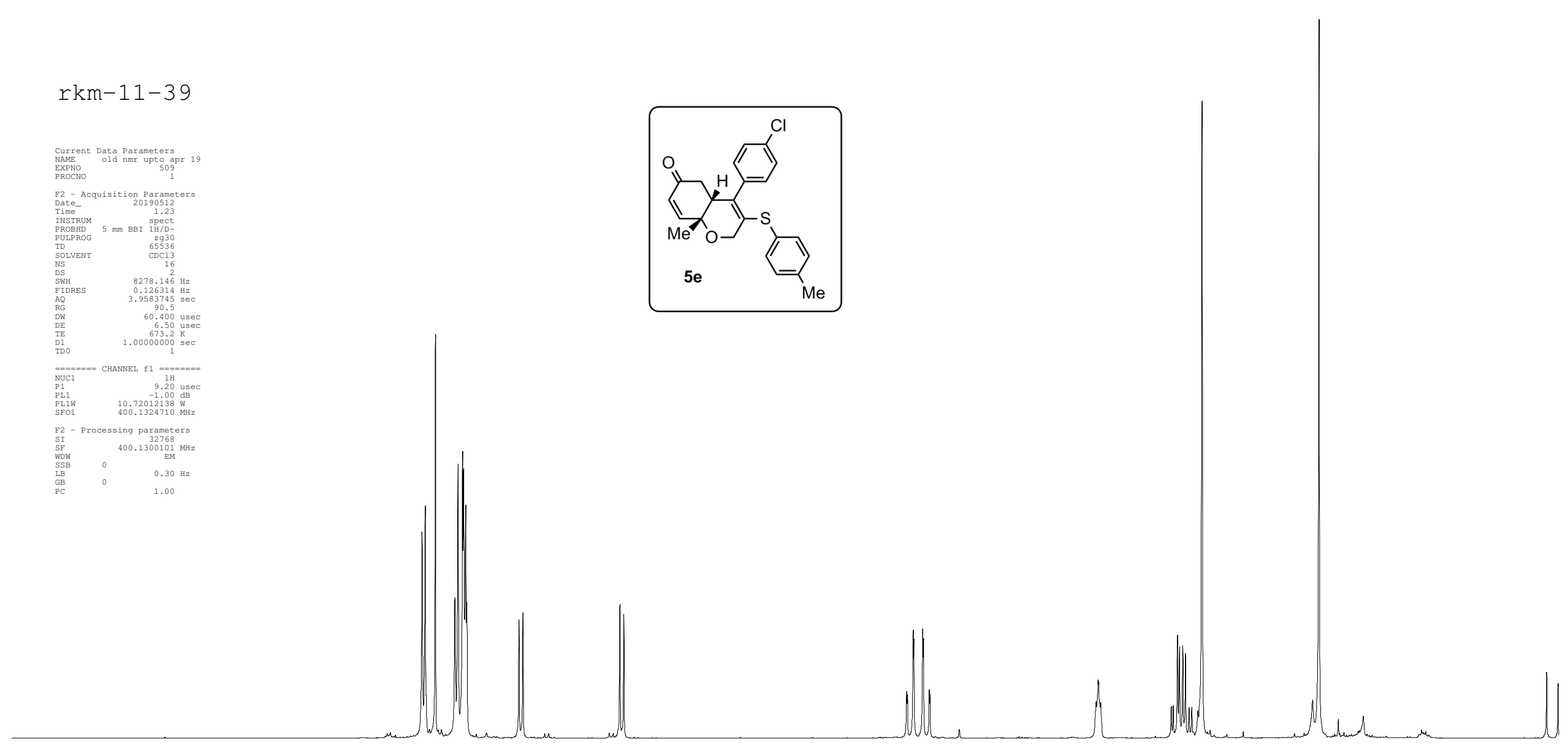

\begin{tabular}{|c|c|c|c|c|c|c|c|c|c|c|c|c|c|c|c|c|c|c|}
\hline & & $T$ & $T$ & & $T$ & $T$ & 1 & $T$ & 1 & 1 & 11 & 1 & 1 & 11 & 1 & 1 & 1 & רי \\
\hline 9.5 & 9.0 & 8.5 & 8.0 & 7.5 & 7.0 & 6.5 & 6.0 & 5.5 & 5.0 & 4.5 & 4.0 & 3.5 & 3.0 & 2.5 & 2.0 & 1.5 & 1.0 & ppm \\
\hline & & & & & 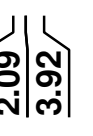 & | & $\left|\begin{array}{l}\hat{\sigma} \\
0 \\
0\end{array}\right|$ & & & & $\mid$\begin{tabular}{c|c}
$\mid$ \\
\\
\end{tabular} & & $\mid$ & $\mid \begin{array}{l}\text { \& } \\
\text { N }\end{array}$ & & 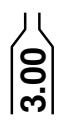 & & \\
\hline
\end{tabular}



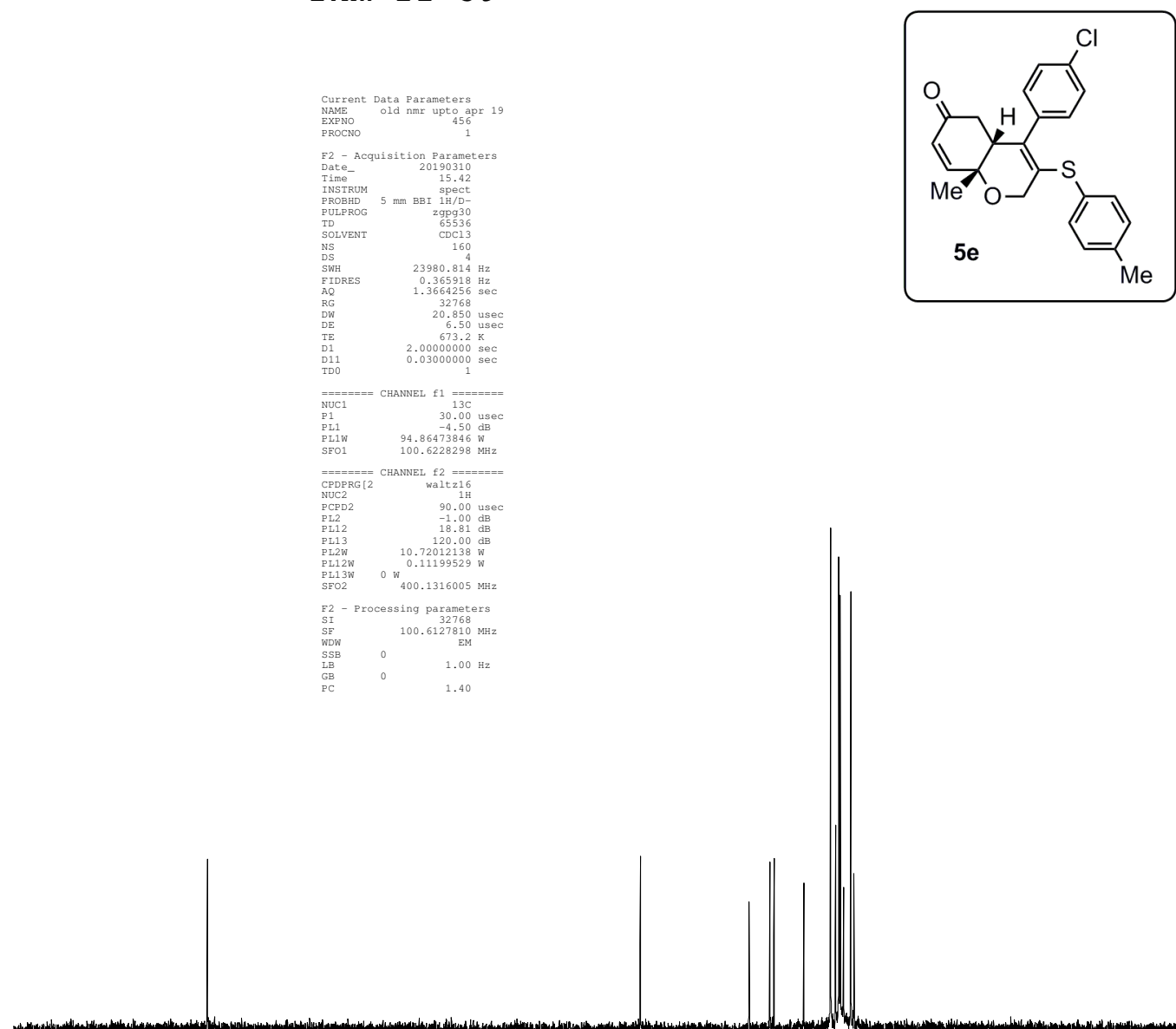

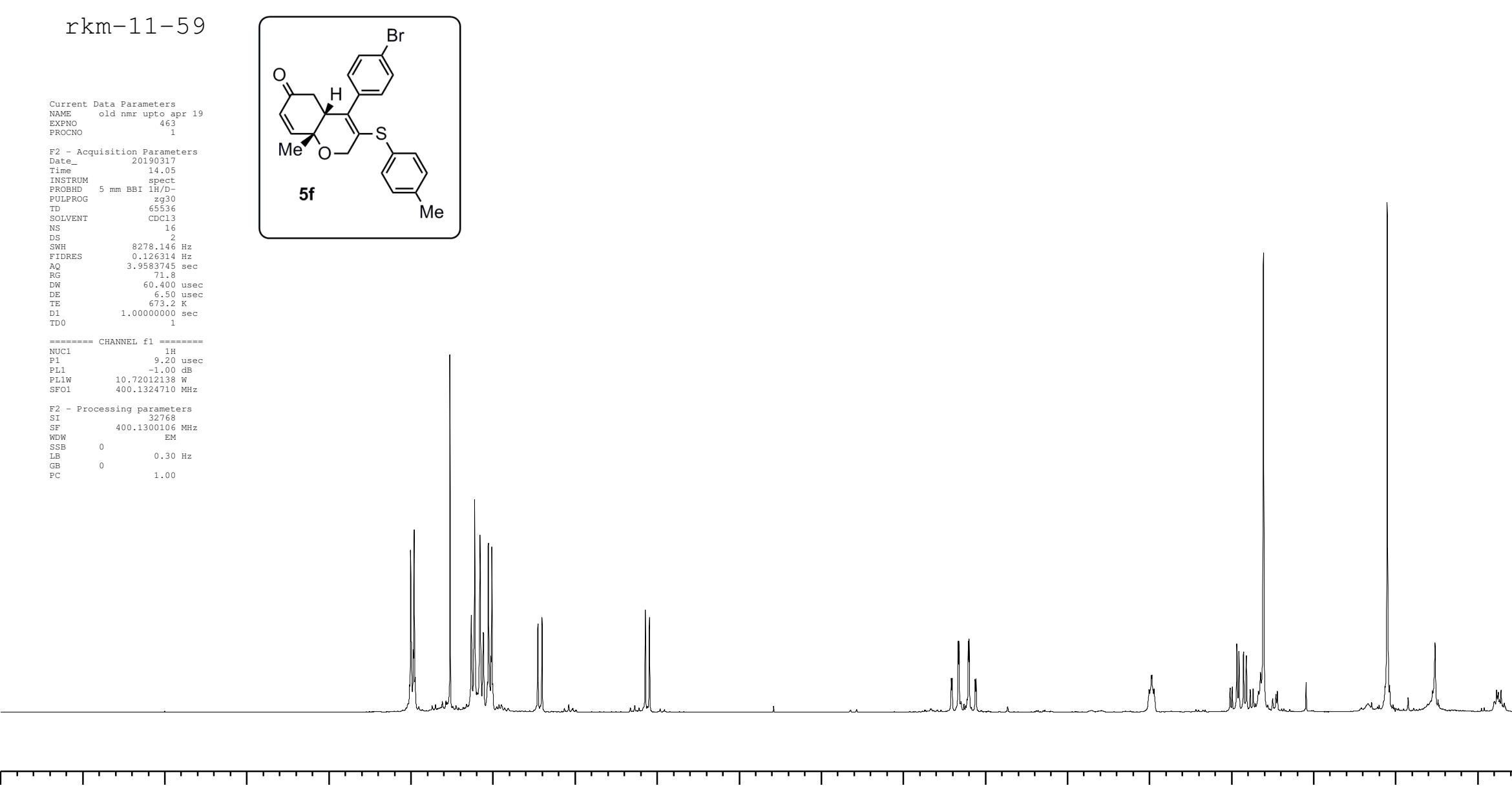

$\begin{array}{llllll}9.5 & 9.0 & 8.5 & 8.0 & 7.5 & 7.0\end{array}$

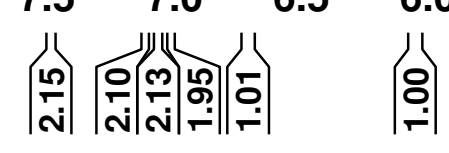
(울| | ppm 

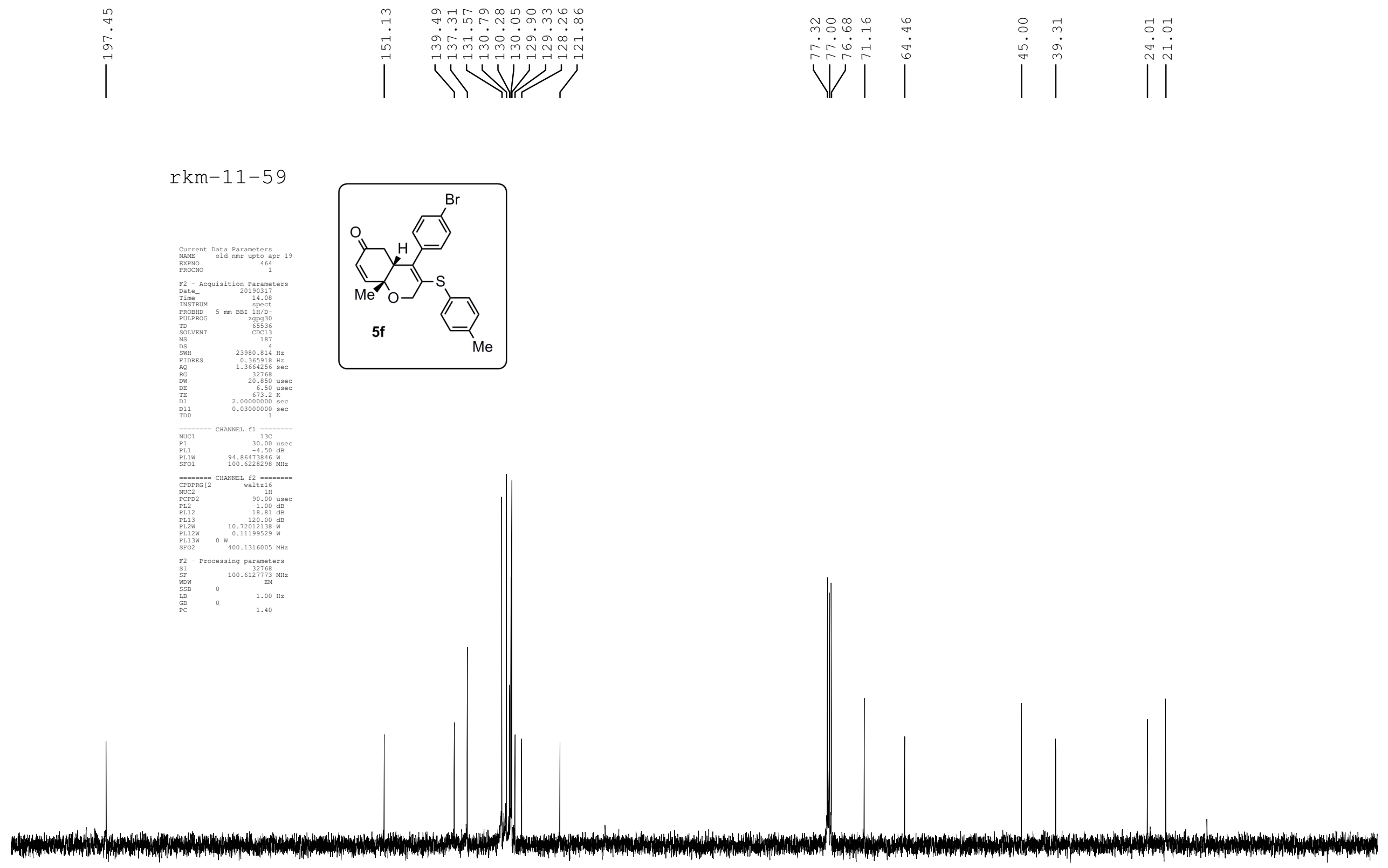
$m \curvearrowleft \hat{m}$ の

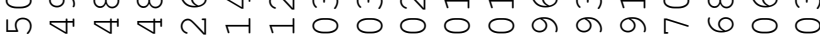

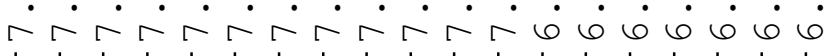

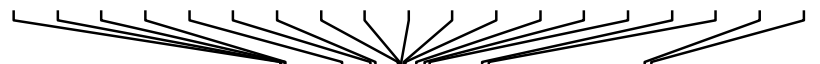

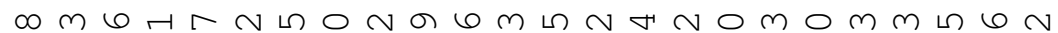

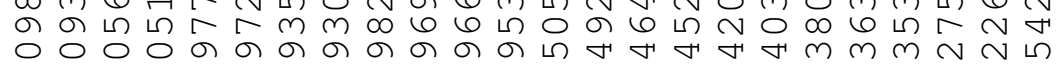
$\dot{\sigma} \dot{\sigma} \dot{\sigma} \dot{\sim} \dot{m} \dot{m} \dot{m} \dot{\sim} \dot{\sim} \dot{\sim} \dot{\sim} \dot{\sim} \dot{\sim} \dot{\sim} \dot{\sim} \dot{\sim} \dot{\sim} \dot{\sim} \dot{\sim} \dot{\sim} \dot{\sim}$

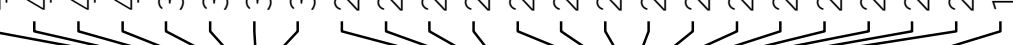

$r k m-11-62$
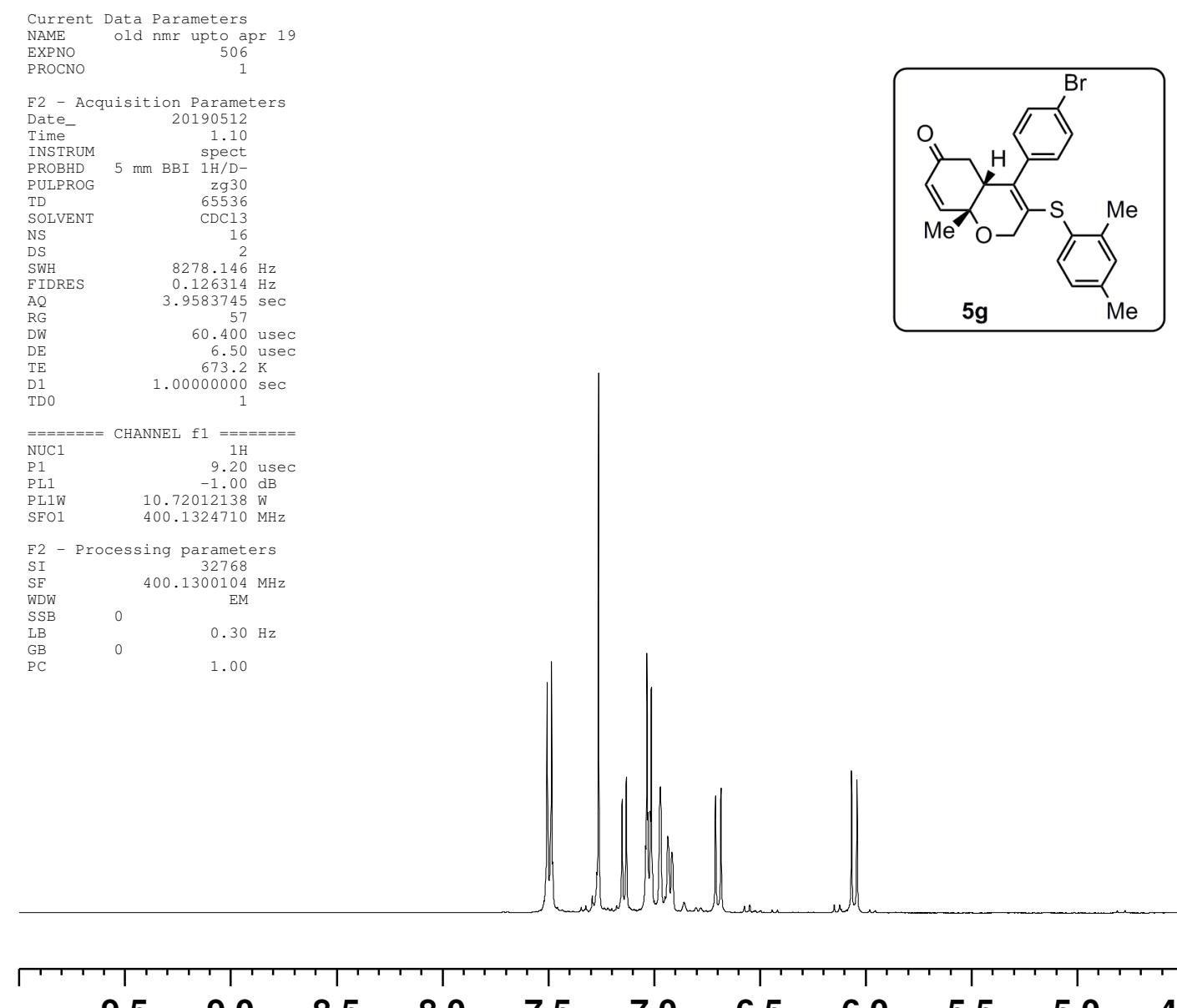

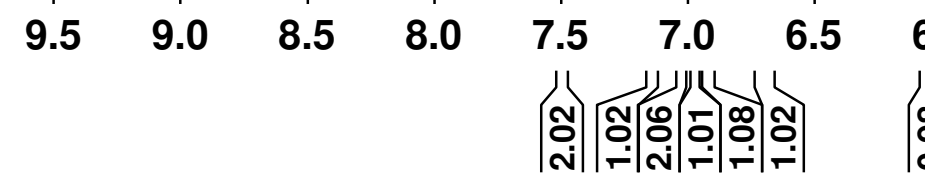

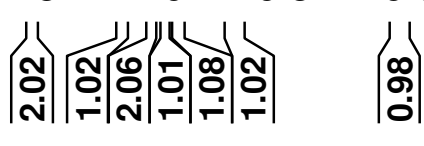
$\left|\begin{array}{l}6.0 \\ \infty \\ 0 \\ 0 \\ 0\end{array}\right|$

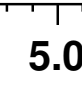

5.0

4.5

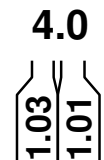



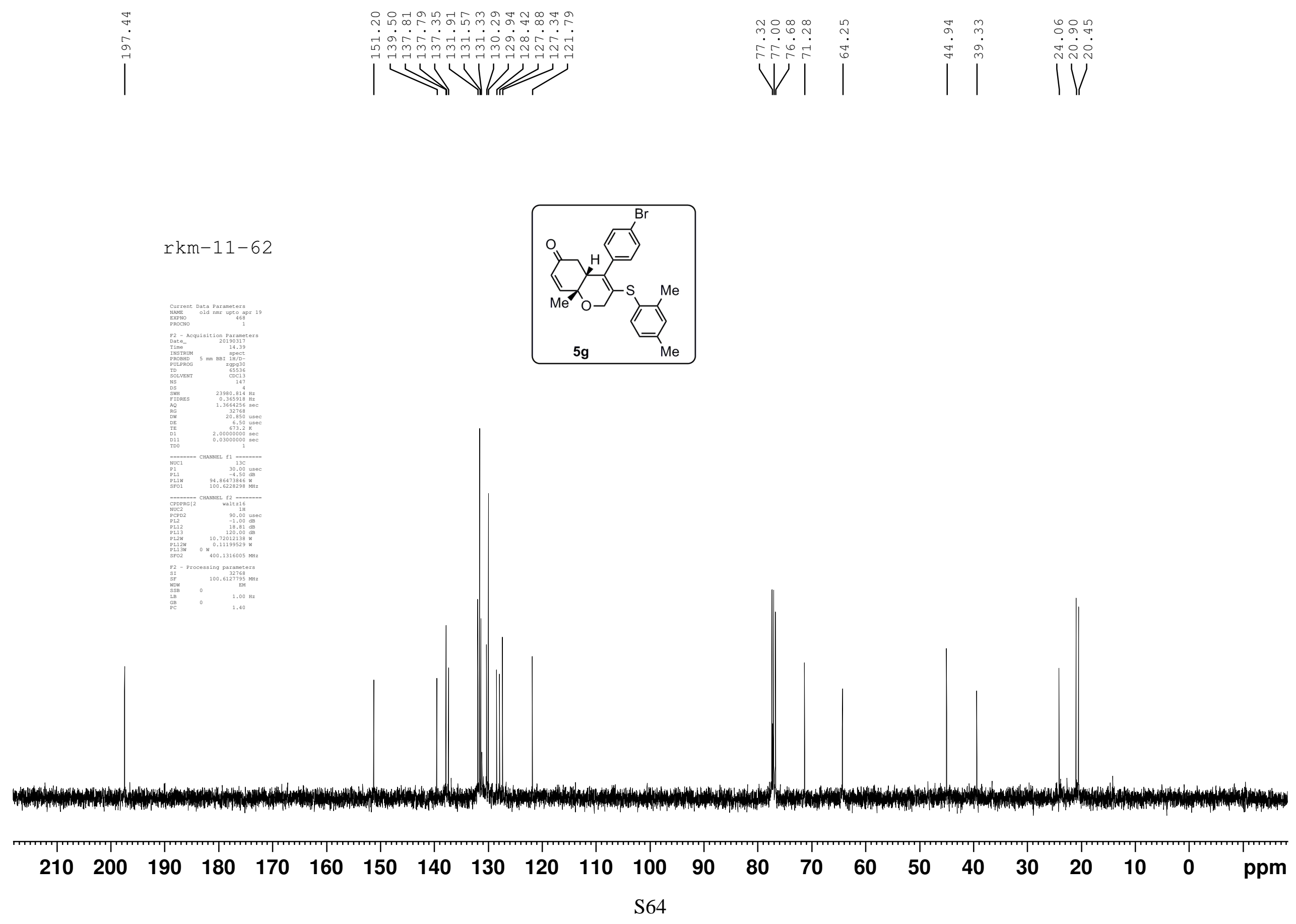

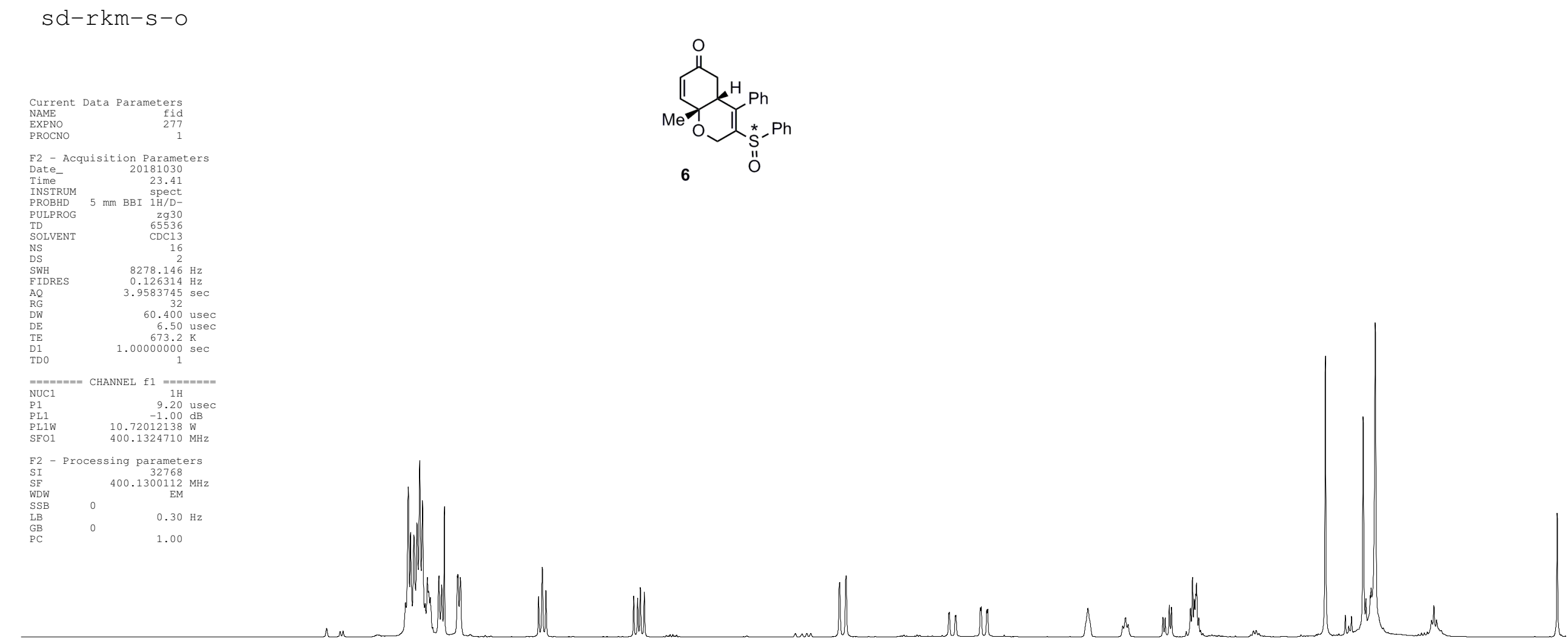

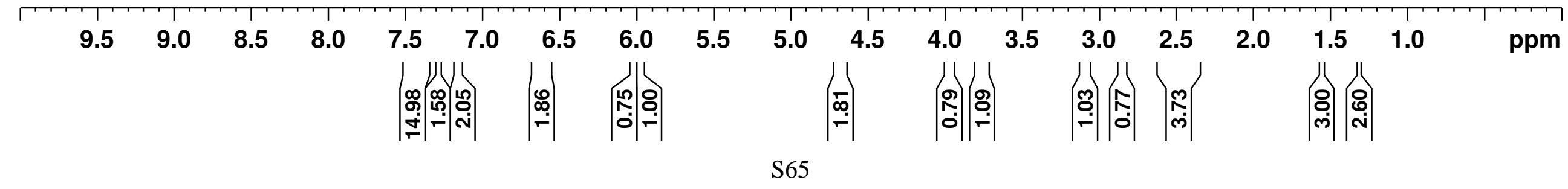



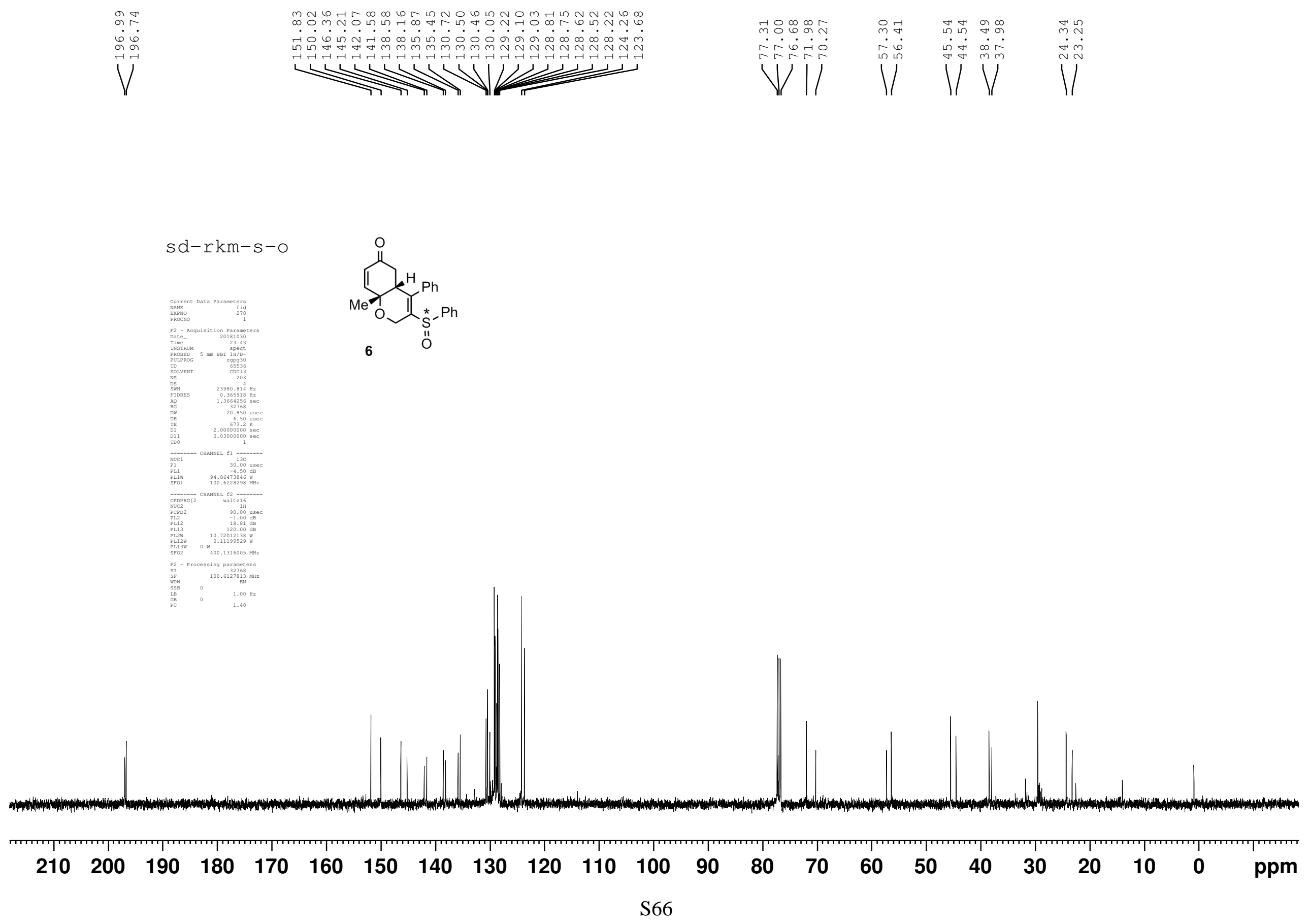


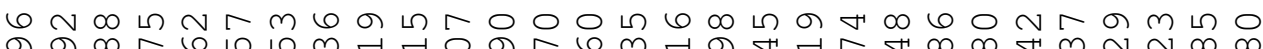
๙

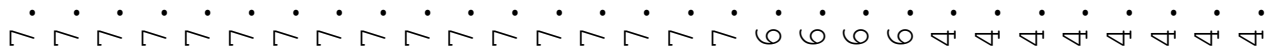

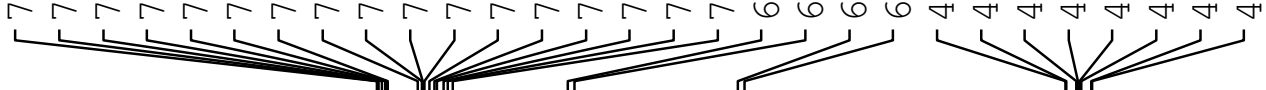

$\mathrm{sd}-\mathrm{rkm}-\mathrm{s} \circ 2$
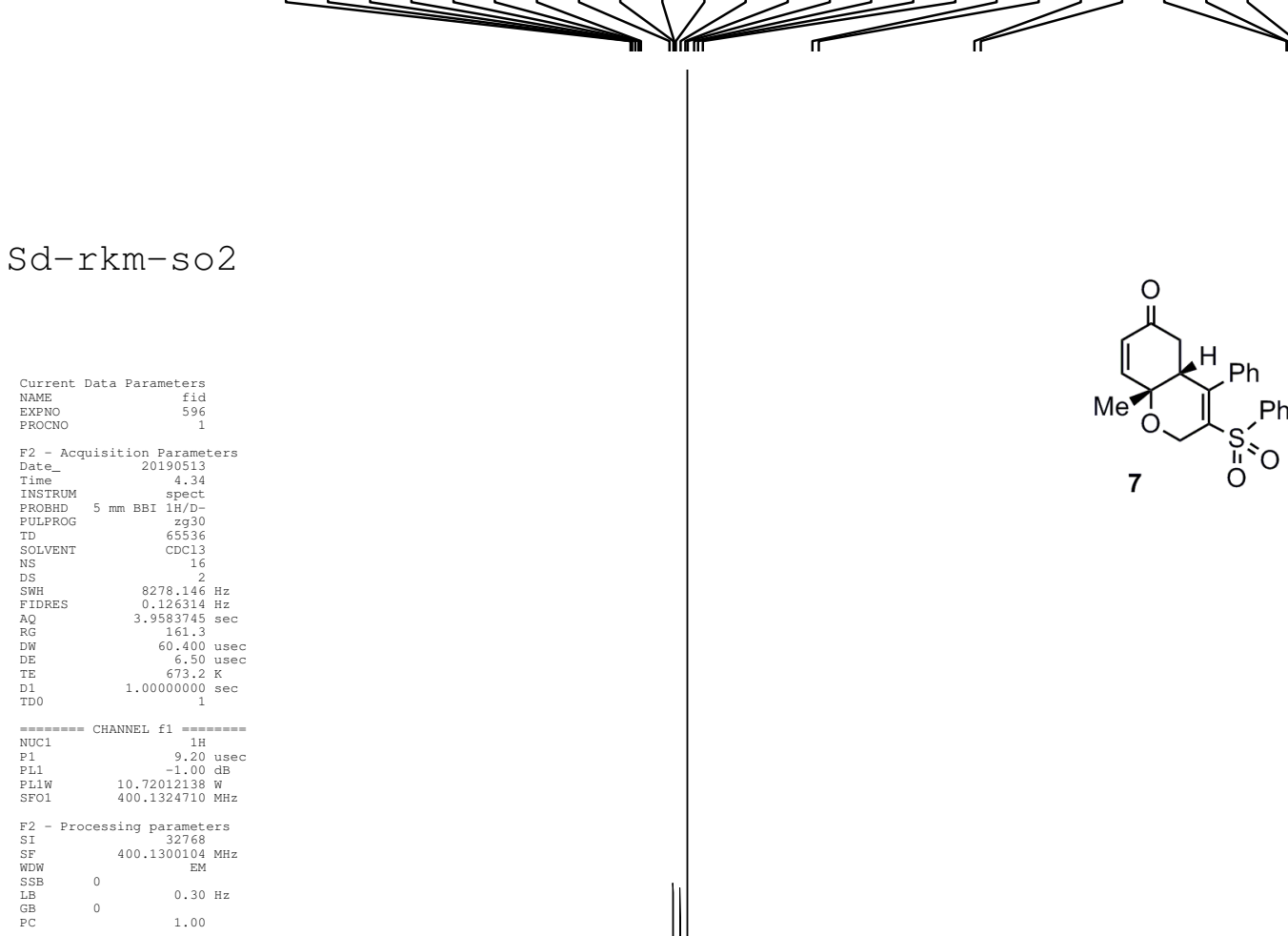

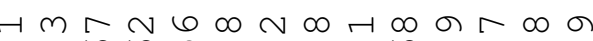

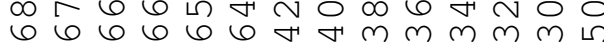
$\dot{\sim} \dot{\sim} \dot{\sim} \dot{\sim} \dot{\sim} \dot{\sim} \dot{\sim} \dot{\sim} \dot{\sim} \dot{\sim} \dot{\sim} \dot{r}$
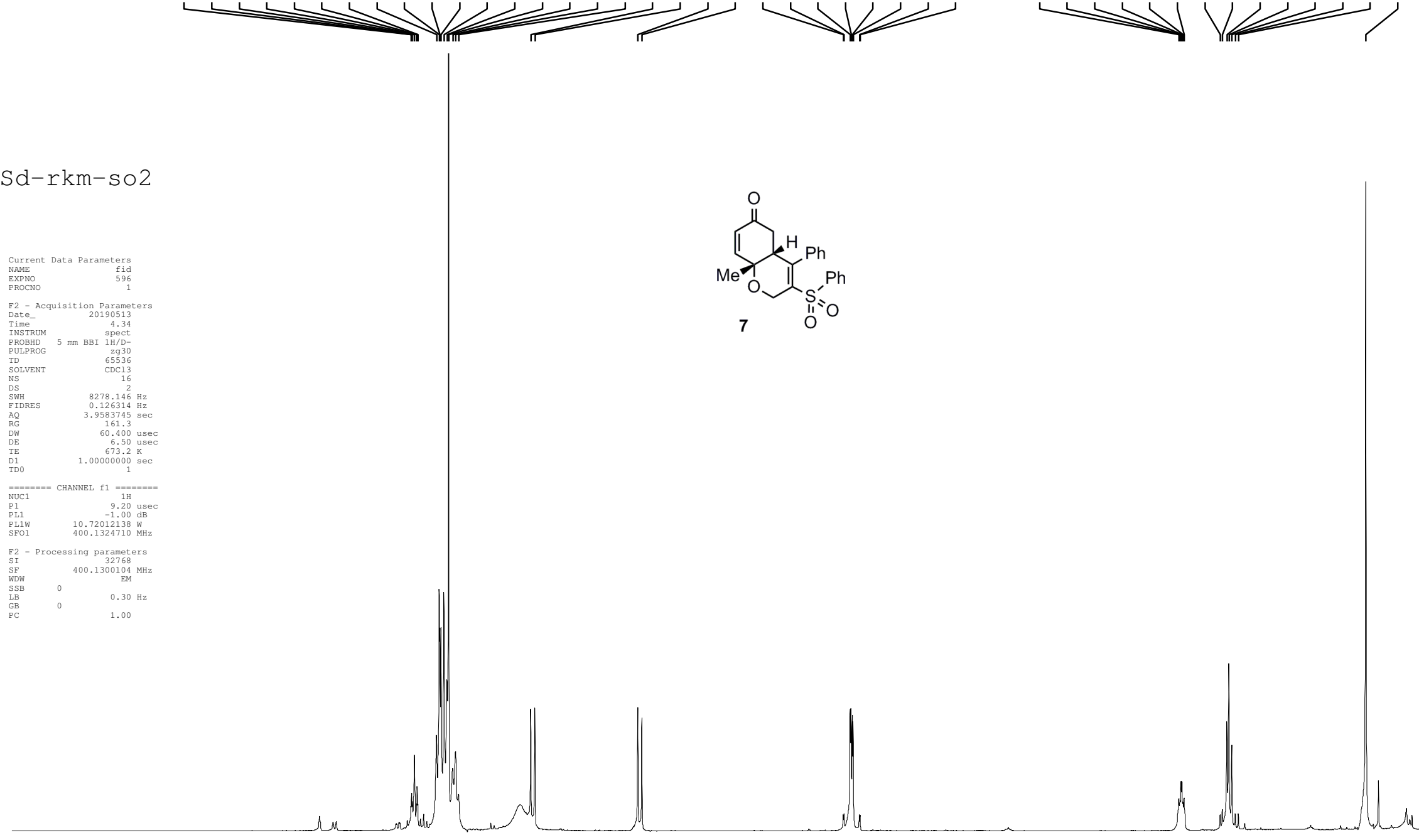

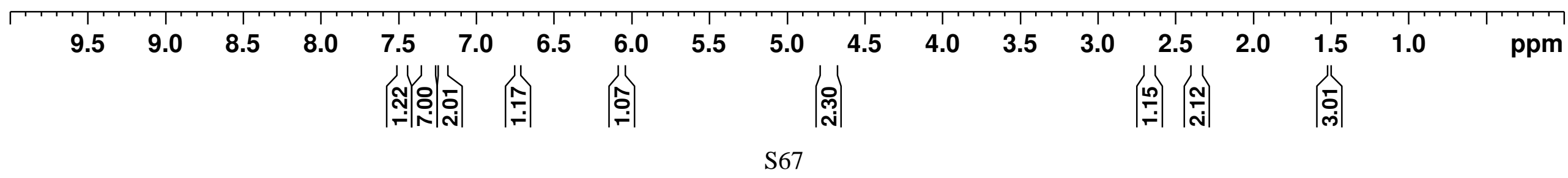




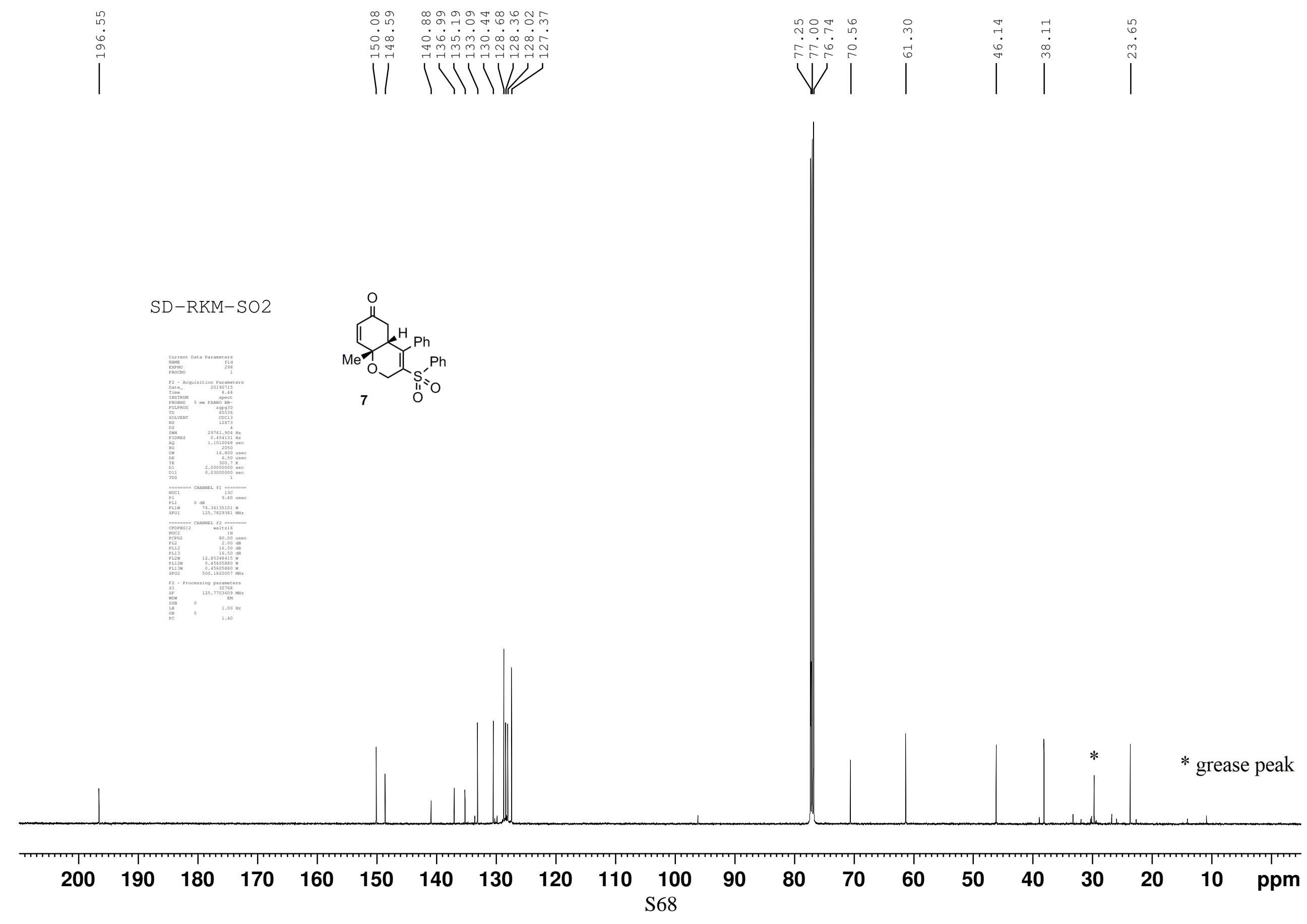



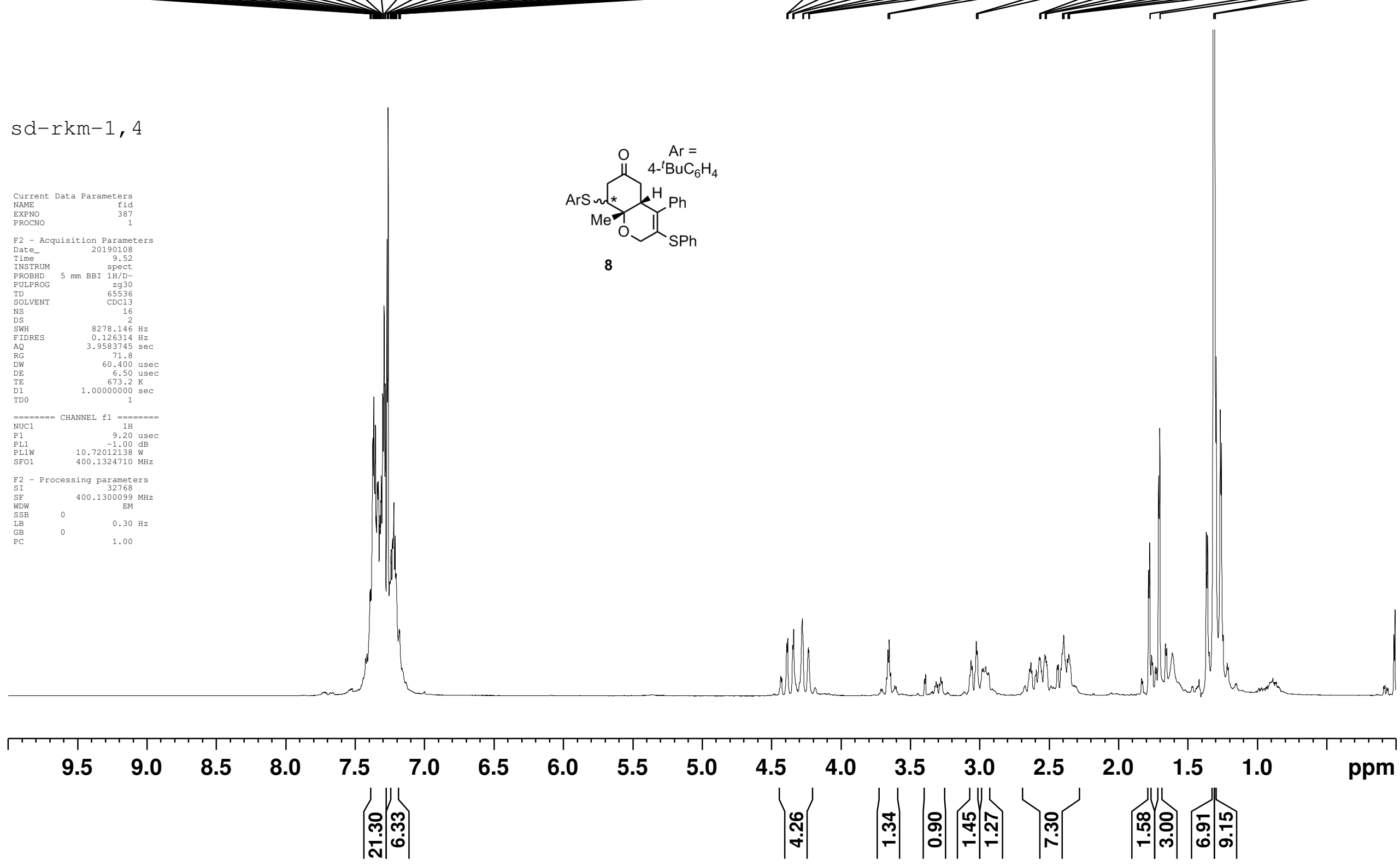


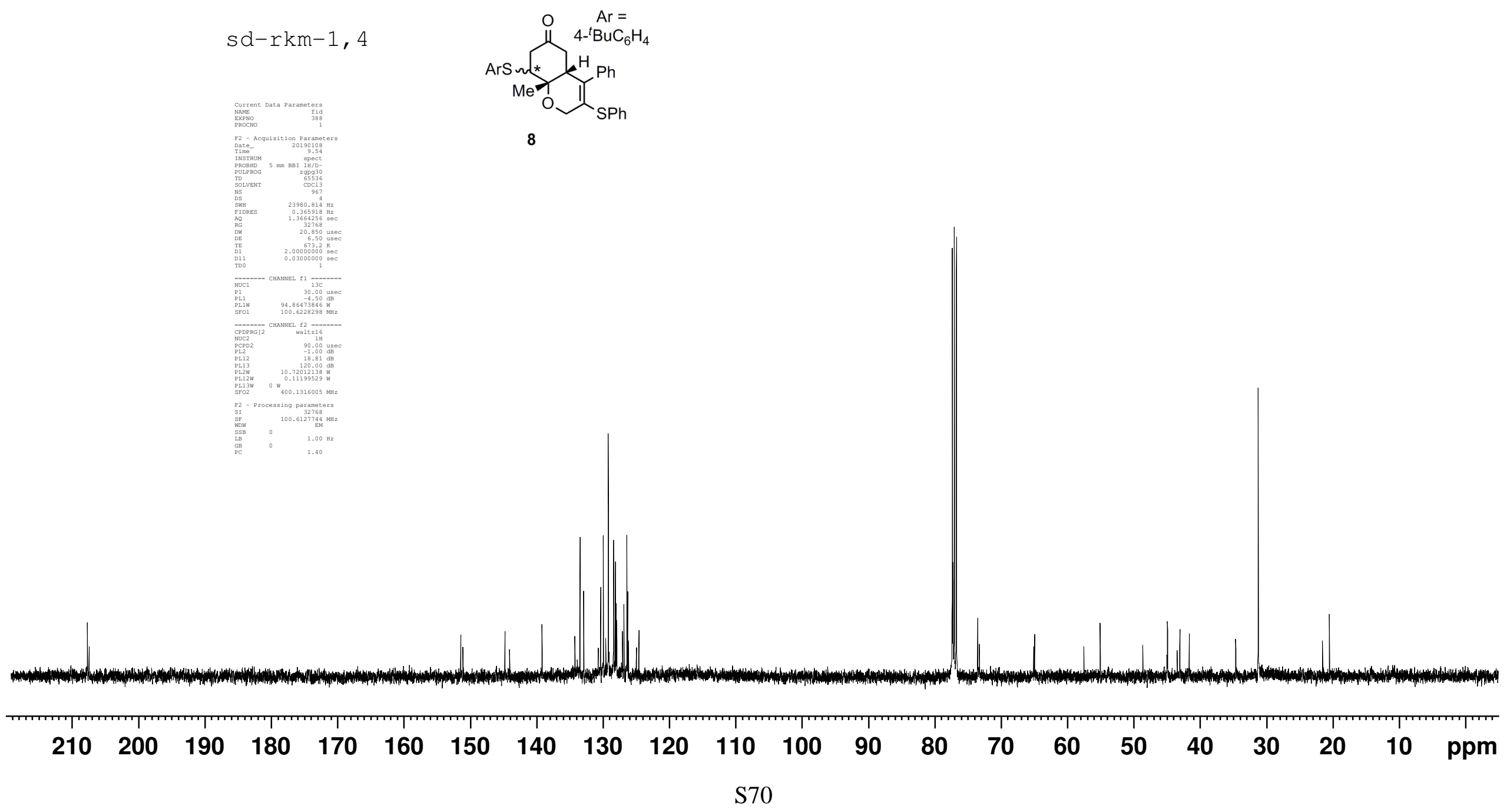



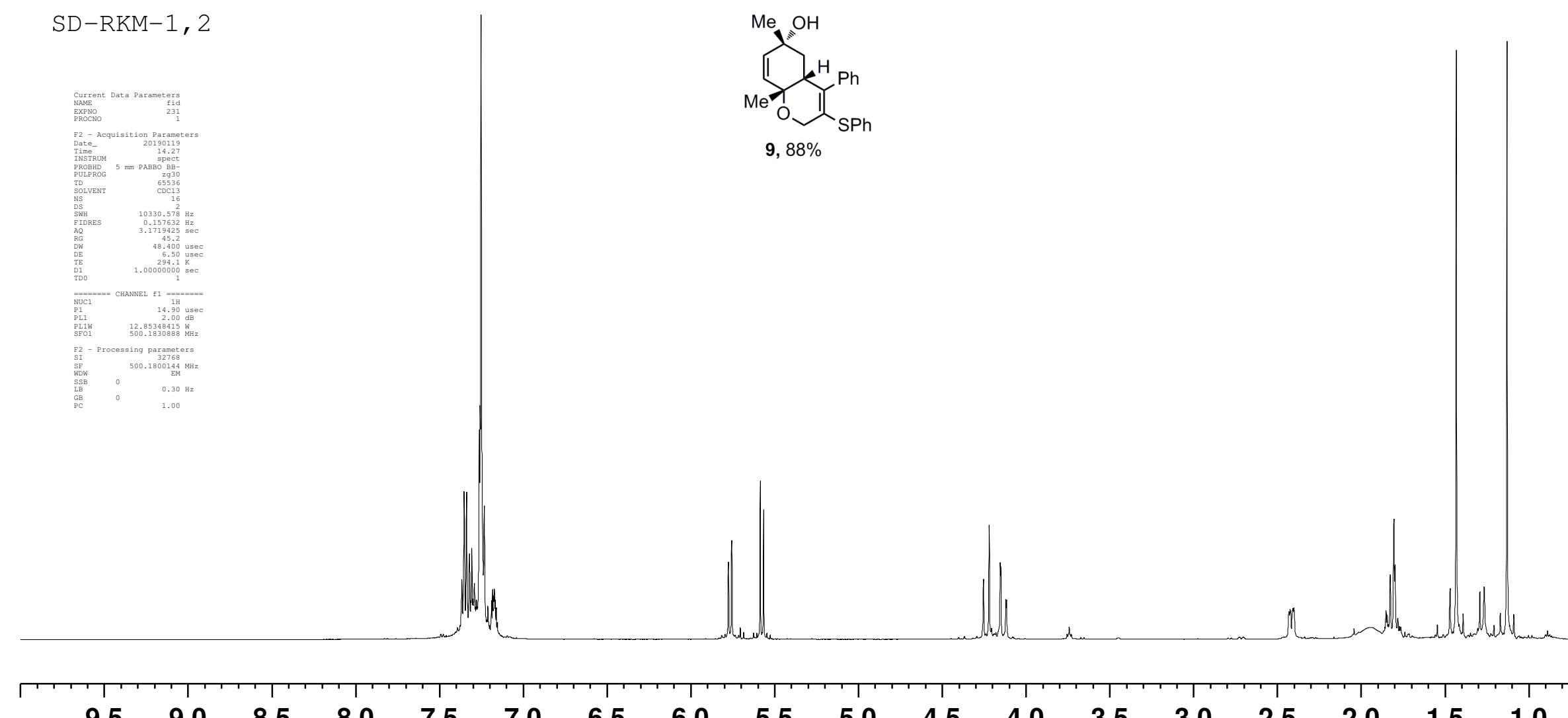

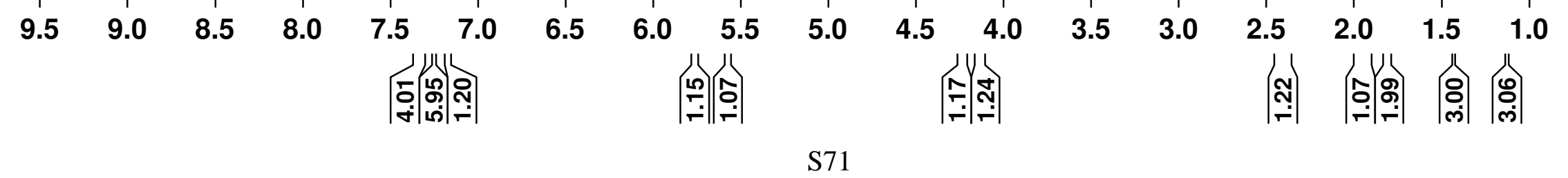



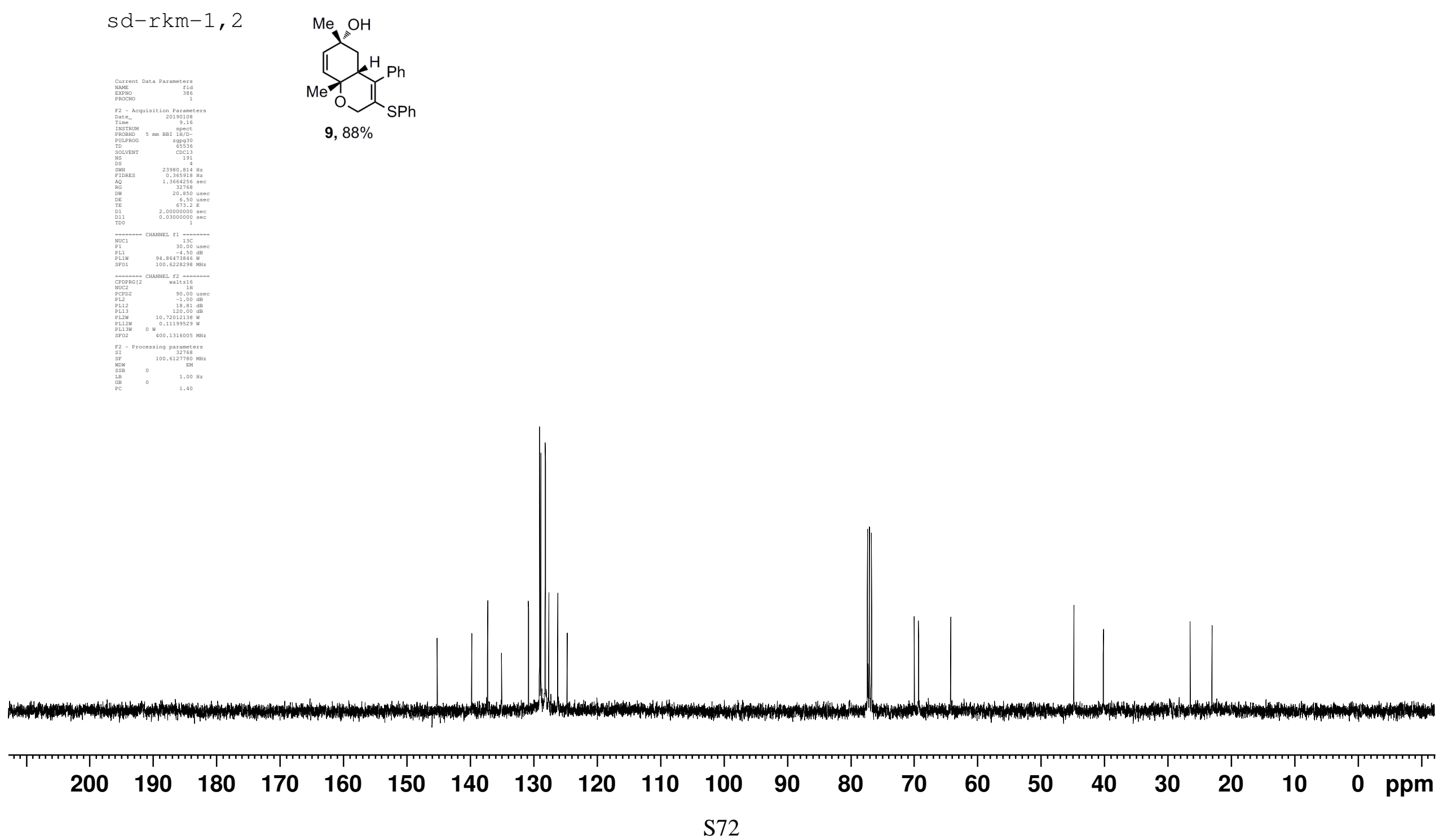


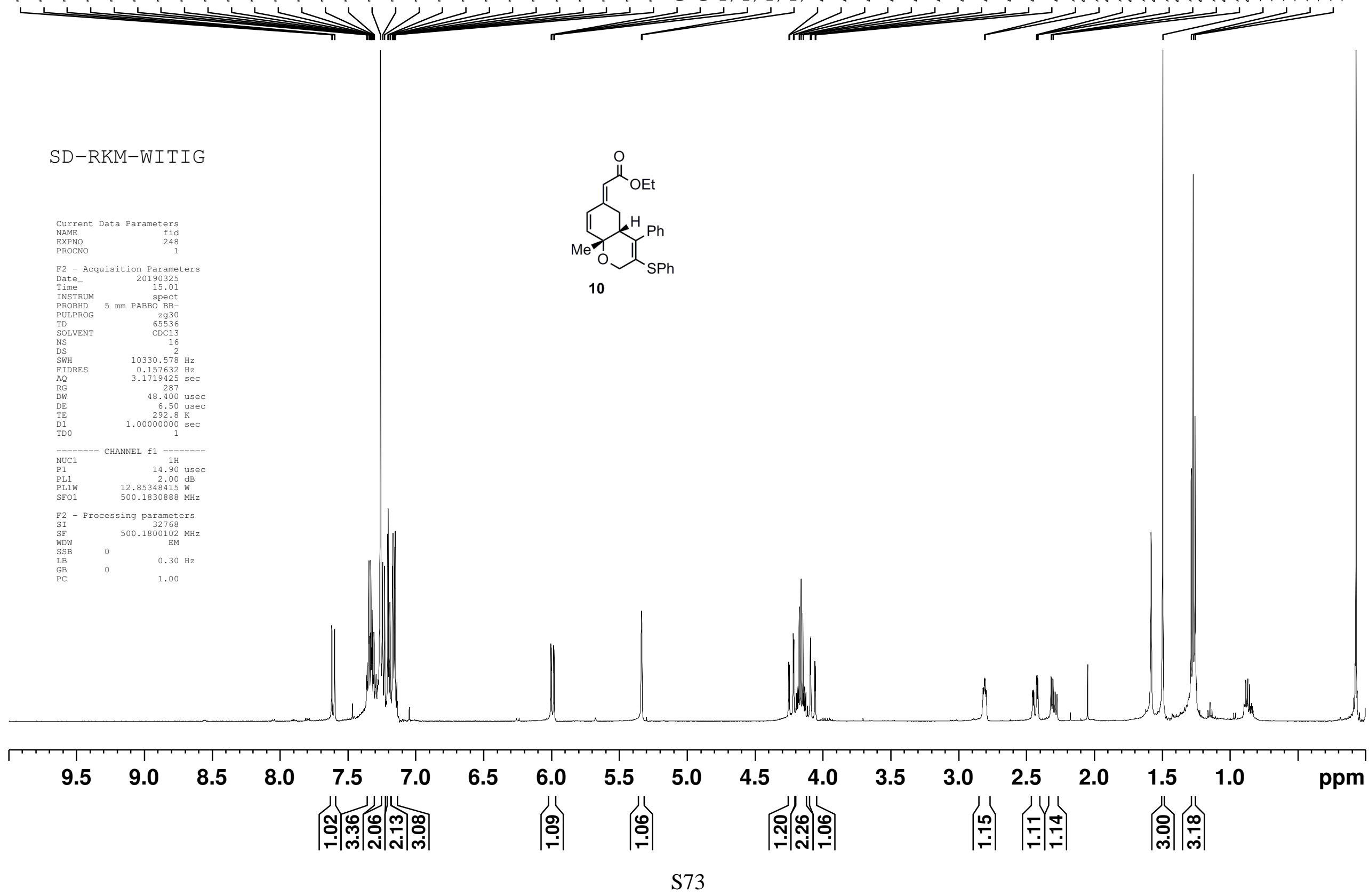




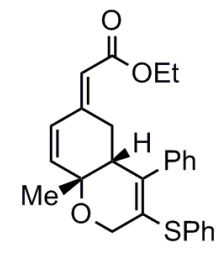

10

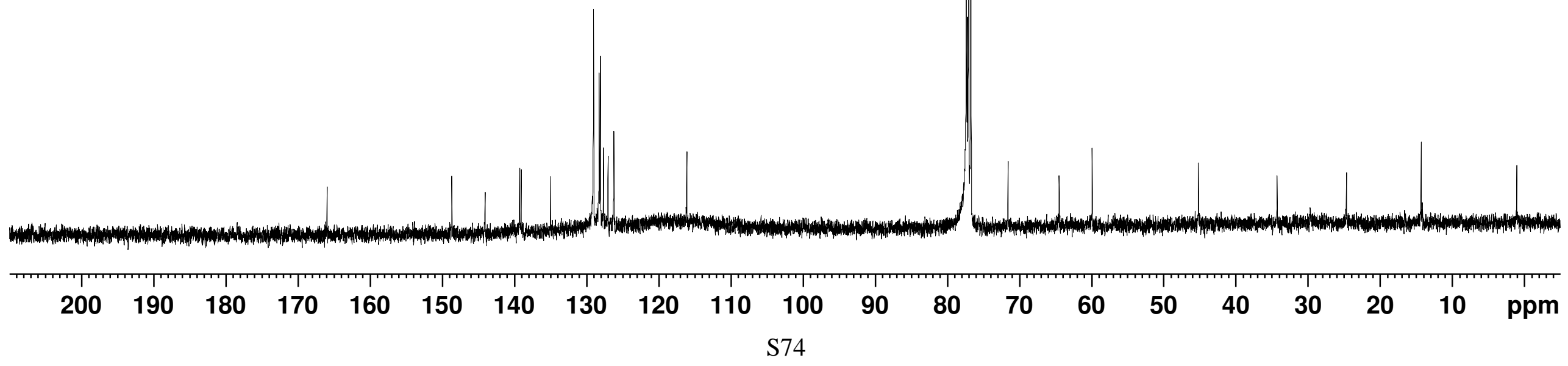








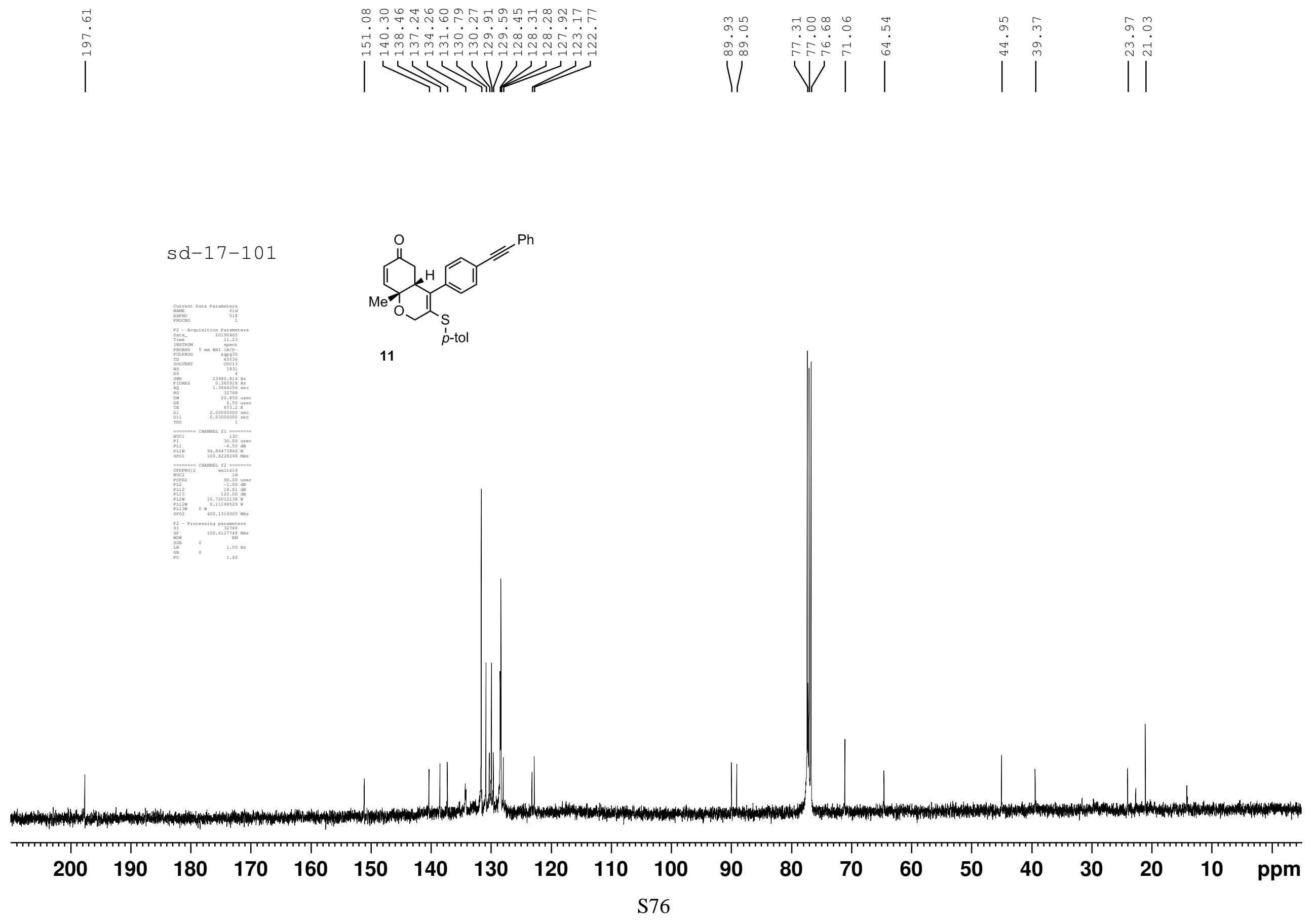